ESAIM: M2AN 50 (2016) 499-539

DOI: $10.1051 / \mathrm{m} 2 \mathrm{an} / 2015057$
ESAIM: Mathematical Modelling and Numerical Analysis

www.esaim-m2an.org

\title{
ON THE CONVERGENCE RATE OF FINITE DIFFERENCE METHODS FOR DEGENERATE CONVECTION-DIFFUSION EQUATIONS IN SEVERAL SPACE DIMENSIONS
}

\author{
Kenneth Hvistendahl Karlsen ${ }^{1}$, Nils Henrik Risebro ${ }^{1}$ \\ AND ERLEND BRiseid StorrøSTEN ${ }^{1}$
}

\begin{abstract}
We analyze upwind difference methods for strongly degenerate convection-diffusion equations in several spatial dimensions. We prove that the local $L^{1}$-error between the exact and numerical solutions is $\mathcal{O}\left(\Delta x^{2 /(19+d)}\right)$, where $d$ is the spatial dimension and $\Delta x$ is the grid size. The error estimate is robust with respect to vanishing diffusion effects. The proof makes effective use of specific kinetic formulations of the difference method and the convection-diffusion equation. This paper is a continuation of [K.H. Karlsen, N.H. Risebro E.B. Storrøsten, Math. Comput. 83 (2014) 2717-2762], in which the one-dimensional case was examined using the Kružkov-Carrillo entropy framework.
\end{abstract}

Mathematics Subject Classification. 65M06, 65M15, 35K65, 35L65.

Received January 27, 2015. Revised July 10, 2015.

Published online March 11, 2016.

\section{INTRODUCTION}

The design of numerical methods for convection-diffusion problems is important for many applications in science and engineering. It is especially challenging to construct accurate methods for nonlinear problems in which the "diffusion part" is small or vanishing, relative to the "convection part" of the problem. Connected to this is the difficult problem of deriving error estimates for numerical methods that are robust in the singular limit as the diffusion coefficient vanishes, thereby avoiding the usual exponential growth of error constants.

In this paper we are interested in deriving error estimates for a class of finite difference methods for nonlinear, possibly strongly degenerate, convection-diffusion problems of the form

$$
\begin{cases}\partial_{t} u+\nabla \cdot f(u)=\Delta A(u), & (t, x) \in \Pi_{T}, \\ u(0, x)=u_{0}(x), & x \in \mathbb{R}^{d},\end{cases}
$$

where $\Pi_{T}=(0, T) \times \mathbb{R}^{d}, T>0, d \geq 1$, and $u: \Pi_{T} \rightarrow \mathbb{R}$ is the unknown function that is sought. The initial datum $u_{0}$ is an integrable and bounded function, while the flux function $f: \mathbb{R} \rightarrow \mathbb{R}^{d}$ and the diffusion function

\footnotetext{
Keywords and phrases. Degenerate convection-diffusion equations, entropy conditions, finite difference methods, error estimates.

1 Department of Mathematics, University of Oslo, P.O. Box 1053, Blindern, 0316 Oslo, Norway.

kennethk@math.uio.no; nilshr@math.uio.no; erlenbs@math.uio.no
} 
$A: \mathbb{R} \rightarrow \mathbb{R}$ satisfy the standing assumptions

$$
f, A \text { locally } C^{1} ; A(0)=0 ; A \text { is nondecreasing. }
$$

By strongly degenerate it is meant that we allow for $A^{\prime}(u)=0$ for all $u$ in some interval $[\alpha, \beta] \subset \mathbb{R}$. The resulting class of equations therefore contains parabolic and hyperbolic equations, as well as a mix thereof. In the nondegenerate (uniformly parabolic) case $A^{\prime}(\cdot)>0$, it is well-known that (1.1) admits a unique classical solution. On the other hand, for strongly degenerate equations with discontinuous solutions, the well-posedness is ensured only in a class of weak solutions satisfying an entropy condition. The following result is known: For $u_{0} \in L^{1}\left(\mathbb{R}^{d}\right) \cap L^{\infty}\left(\mathbb{R}^{d}\right)$, there exists a unique solution $u \in C\left((0, T) ; L^{1}\left(\mathbb{R}^{d}\right)\right), u \in L^{\infty}\left(\Pi_{T}\right)$ of (1.1) such that $\partial_{x} A(u) \in L^{2}\left(\Pi_{T}\right)$ and for all convex functions $S$ with $q_{S}^{\prime}=S^{\prime} f^{\prime}$ and $r_{S}^{\prime}=S^{\prime} A^{\prime}$,

$$
\partial_{t} S(u)+\nabla \cdot q_{S}(u)-\Delta r_{S}(u) \leq 0, \quad \text { weakly on }[0, T) \times \mathbb{R}^{d} .
$$

These inequalities are referred to as entropy inequalities and the corresponding solution is called an entropy solution.

For conservation laws $\left(A^{\prime} \equiv 0\right)$, the well-posedness of entropy solutions is a celebrated result due to Kružkov [26]. Carrillo [8] extended this result to degenerate parabolic problems such as (1.1). For uniqueness of entropy solutions in the $B V$ class, see $[35,36]$. An alternative well-posedness theory, based on the so-called kinetic formulation, was developed by Lions, Perthame, and Tadmor [29] and Chen and Perthame [10]. We refer to $[2,16]$ for an overview of the relevant literature on hyperbolic and mixed hyperbolic-parabolic problems.

In this paper we derive error estimates for numerical approximations of entropy solutions to convectiondiffusion equations. Convergence results, without error estimates, have been obtained for difference methods $[17,18,23]$; finite volume methods [1,21]; splitting methods [22]; and BGK approximations [3,6], to mention a few references. For a posteriori error estimates for finite volume methods, see [31].

We are herein interested in estimating the error committed by a class of monotone difference methods. The monotone methods make use of an upwind discretization of the convection term and a centred discretization of the parabolic term. For notational simplicity in the introduction, let us assume $f^{i, \prime}(\cdot) \geq 0$ and consider the prototype (semi-discrete) difference method

$$
\frac{\mathrm{d}}{\mathrm{d} t} u_{\alpha}+\sum_{i=1}^{d} \frac{f^{i}\left(u_{\alpha}\right)-f^{i}\left(u_{\alpha-e_{i}}\right)}{\Delta x}=\sum_{i=1}^{d} \frac{A\left(u_{\alpha+e_{i}}\right)-2 A\left(u_{\alpha}\right)+A\left(u_{\alpha-e_{i}}\right)}{\Delta x^{2}},
$$

where $\alpha=\left(\alpha_{1}, \ldots, \alpha_{d}\right) \in \mathbb{Z}^{d}$ is a multi-index, $e_{i}$ is the $i$ th unit vector in $\mathbb{R}^{d}$, and $\Delta x>0$ is the spatial grid size. Although our methods are semi-discrete, i.e., not discretized in time, the results and proofs can be adjusted to cover some fully discrete methods, such as the implicit method analyzed in [18]. We refer to [25] for a discussion of this topic when $d=1$.

Denote by $u_{\Delta x}$ the piecewise constant interpolant linked to $u_{\alpha}$. The goal is to determine a number (convergence rate) $\gamma>0$ such that

$$
\left\|u_{\Delta x}(t, \cdot)-u(t, \cdot)\right\|_{L^{1}} \leq C \Delta x^{\gamma}, \quad\left(u_{0} \in B V\right),
$$

for some constant $C>0$ independent of $\Delta x$ and (the smallness of) $A^{\prime}$.

In the purely hyperbolic case $\left(A^{\prime} \equiv 0\right)$, a prominent result due to Kuznetsov [28] says that $\gamma$ is $1 / 2$ for monotone difference methods, as well as for the vanishing viscosity method. Influenced by [28], a number of works have further developed the "Kružkov-Kuznetsov" error estimation theory for conservation laws. We refer to $[5,13]$ for an overview of the relevant results. Making use of the kinetic formulation, Perthame [33] provided an alternative route to error estimates.

With regard to convection-diffusion equations $(1.1)$ with $A^{\prime}(\cdot) \geq 0$, the subject of error estimates is significantly more difficult. It is only recently that there has been some progress. The simplest case is the vanishing viscosity method. Denote by $u^{\eta}$ the solution of the uniformly parabolic equation

$$
u_{t}^{\eta}+\nabla \cdot f\left(u^{\eta}\right)=\Delta A^{\eta}\left(u^{\eta}\right), \quad A^{\eta}(u)=A(u)+\eta u,
$$


where $\eta>0$ is a (small) viscosity parameter. We have the following error estimate for the viscosity approximation $u^{\eta}$ :

$$
\left\|u^{\eta}(\cdot, t)-u(\cdot, t)\right\|_{L^{1}} \leq C \sqrt{\eta}, \quad\left(u_{0} \in B V\right),
$$

where $u$ is the entropy solution of (1.1). A "Kružkov-Kuznetsov" type proof of this result is given in [19], see also [20] for a boundary value problem. The error bound (1.5) can also be seen as an outcome of continuous dependence estimates $[11,13]$ or the kinetic formulation $[12,30]$.

For conservation laws, the error estimate (1.5) for the viscous equation reveals what to expect for monotone difference methods [28]. This suggestive link breaks down for degenerate convection-diffusion equations (1.1), $c f$. [25], a fact that may foreshadow added difficulties coming from a second order operator. Indeed, for general $A$ satisfying (1.2) and in one spatial dimension, the work [24] established (1.3) with $\gamma=1 / 11$, a rate that was recently improved to $\gamma=1 / 3$ in [25]. Although $\gamma=1 / 3$ is the best available rate at the moment, its optimality is unknown and also far from the convergence rate $\gamma=1 / 2$ known to be optimal for conservation laws. But in spite of that, with a linear diffusion function $A$, the convergence rate improves to $\gamma=1 / 2[24,25]$.

Apart from a result $(\gamma=1 / 2)$ for linear convection-diffusion equations [12], we are not aware of any results for multi-dimensional equations (1.1) with a degenerate, nonlinear diffusion part. In this paper we establish (1.3) with

$$
\gamma=\frac{2}{19+d} \quad(d \text { is the spatial dimension })
$$

for general diffusion functions $A$ obeying (1.2).

A technical aspect of the proof of (1.3) is that we are not applying the difference method directly to (1.1) but rather to (1.4). Denoting the corresponding numerical solution by $u_{\Delta x}^{\eta}$, we will prove that (1.3) holds with $u_{\Delta x}, u$ replaced by $u_{\Delta x}^{\eta}, u^{\eta}$, respectively, and that the error constant $C$ is not depending on the parameter $\eta$. Our original claim (1.3) follows from this, since we have the error estimate (1.5).

To help motivate the technical arguments coming later, let us lay out a "high-level" overview of the analysis and some of the difficulties involved. As just alluded to, we will mostly work under the assumption $A^{\prime}>0$. As a consequence no information is lost upon working with $A(u)$ instead of $u$ in the kinetic formulation (compare with the $u$-based formulation in [10]). Set $B=A^{-1}$ and define $g$ by $g \circ A=f$. Then the solution $u$ of (1.1) satisfies

$$
B^{\prime}(\zeta) \partial_{t} \chi_{A(u)}+g^{\prime}(\zeta) \cdot \nabla \chi_{A(u)}-\Delta \chi_{A(u)}=\partial_{\zeta} m_{A(u)}
$$

where

$$
\begin{aligned}
& m_{A(u)}=m_{A(u)}(\zeta)=\delta(\zeta-A(u))|\nabla A(u)|^{2}, \\
& \chi_{A(u)}=\chi_{A(u)}(\zeta)= \begin{cases}1 & \text { if } 0<\zeta \leq A(u), \\
-1 & \text { if } A(u) \leq \zeta<0, \\
0 & \text { otherwise }\end{cases}
\end{aligned}
$$

This new formulation, although restricted to nondegenerate (isotropic) diffusion, allows for a simpler proof of the $L^{1}$ contraction property and thus the error estimate (1.3). More specifically, certain error terms linked to the regularization of the $\chi$ function [10] can be avoided, a fact that we use to our benefit.

Now we indicate how (1.7) leads to the $L^{1}$ contraction property. Let $u$ and $v$ be solutions to (1.1) with initial values $u_{0}$ and $v_{0}$, respectively. Following $[10,33]$, we introduce the microscopic contraction functional

$$
Q_{u, v}(\xi)=\left|\chi_{u}(\xi)\right|+\left|\chi_{v}(\xi)\right|-2 \chi_{u}(\xi) \chi_{v}(\xi) .
$$

Under the change of variable $\zeta=A(\xi)$,

$$
|u-v|=\int_{\mathbb{R}} Q_{u, v}(\xi) \mathrm{d} \xi=\int_{\mathbb{R}} B^{\prime}(\zeta) Q_{A(u), A(v)}(\zeta) \mathrm{d} \zeta,
$$


and hence

$$
\begin{aligned}
\partial_{t}|u-v|= & \int_{\mathbb{R}} B^{\prime}(\zeta) \partial_{t} Q_{A(u), A(v)}(\zeta) \mathrm{d} \zeta \\
= & \int_{\mathbb{R}} B^{\prime}(\zeta) \partial_{t}\left|\chi_{A(u)}(\zeta)\right| \mathrm{d} \zeta+\int_{\mathbb{R}} B^{\prime}(\zeta) \partial_{t}\left|\chi_{A(v)}(\zeta)\right| \mathrm{d} \zeta \\
& -2 \int_{\mathbb{R}} B^{\prime}(\zeta) \partial_{t}\left(\chi_{A(u)}(\zeta) \chi_{A(v)}(\zeta)\right) \mathrm{d} \zeta
\end{aligned}
$$

In view of (1.7), the chain rule yields

$$
B^{\prime}(\zeta) \partial_{t}\left|\chi_{A(u)}\right|+g^{\prime}(\zeta) \cdot \nabla\left|\chi_{(A(u)}\right|-\Delta\left|\chi_{A(u)}\right|=\operatorname{sign}(\zeta) \partial_{\zeta} m_{A(u)},
$$

with an analogous equation for $v$. Using the equations for $\chi_{A(u)}, \chi_{A(v)}$ and Leibniz's product rule, we easily check that

$$
\begin{aligned}
& B^{\prime}(\zeta) \partial_{t}\left(\chi_{A(u)} \chi_{A(v)}\right)+g^{\prime}(\zeta) \cdot \nabla\left(\chi_{A(u)} \chi_{A(v)}\right)-\Delta\left(\chi_{A(u)} \chi_{A(v)}\right) \\
& =\chi_{A(v)} \partial_{\zeta} m_{A(u)}+\chi_{A(u)} \partial_{\zeta} m_{A(v)}-2 \nabla \chi_{A(u)} \cdot \nabla \chi_{A(v)} .
\end{aligned}
$$

Making use of (1.10) and (1.11) in (1.9) yields

$$
\begin{aligned}
\partial_{t}|u-v|= & -\int_{\mathbb{R}} g^{\prime}(\zeta) \cdot \nabla Q_{A(u), A(v)}(\zeta) \mathrm{d} \zeta+\int_{\mathbb{R}} \Delta Q_{A(u), A(v)}(\zeta) \mathrm{d} \zeta \\
& +\int_{\mathbb{R}} D(\zeta) \mathrm{d} \zeta
\end{aligned}
$$

where

$$
\begin{aligned}
D(\zeta)= & \left(\operatorname{sign}(\zeta)-2 \chi_{A(v)}(\zeta)\right) \partial_{\zeta} m_{A(u)}+\left(\operatorname{sign}(\zeta)-2 \chi_{A(u)}(\zeta)\right) \partial_{\zeta} m_{A(v)} \\
& +4 \nabla \chi_{A(u)}(\zeta) \cdot \nabla \chi_{A(v)}(\zeta) \\
= & : D_{1}(\zeta)+D_{2}(\zeta)+D_{3}(\zeta)
\end{aligned}
$$

the term $D(\cdot)$ accounts for the parabolic dissipation effects associated with $u, v$. Integrating (1.12) in $x$ gives

$$
\frac{\mathrm{d}}{\mathrm{d} t} \int|u(t, x)-v(t, x)| \mathrm{d} x=\iint_{\mathbb{R}} D(\zeta) \mathrm{d} \zeta \mathrm{d} x .
$$

Although the computations have been formal up to this point, they are valid when interpreted in the sense of distributions. Moreover, as will be seen later, these computations can with some effort be replicated at the discrete level, i.e., when we replace the function $v$ by the numerical solution $u_{\Delta x}$.

Clearly, the $L^{1}$-contraction property follows if we can confirm that

$$
\int_{\mathbb{R}} D(\zeta) \mathrm{d} \zeta \leq 0
$$

This step is rather delicate and will ask for a regularization of the $\chi$ functions. Indeed, the hard part of the proof leading up to (1.3), (1.6) is related to this step. Let us for the moment ignore the regularization procedure, and continue with formal computations. Note that

$$
\operatorname{sign}(\zeta)-2 \chi_{A(v)}(\zeta)=\operatorname{sign}(\zeta-A(v)),
$$

and thus, after an integration by parts followed by an application of the chain rule,

$$
\int_{\mathbb{R}} D_{1}(\zeta) \mathrm{d} \zeta=-2 \int_{\mathbb{R}} \delta(\zeta-A(u)) \delta(\zeta-A(v))|\nabla A(u)|^{2} \mathrm{~d} \zeta .
$$


Similarly,

$$
\int_{\mathbb{R}} D_{2}(\zeta) \mathrm{d} \zeta=-2 \int_{\mathbb{R}} \delta(\zeta-A(u)) \delta(\zeta-A(v))|\nabla A(v)|^{2} \mathrm{~d} \zeta
$$

Again by the chain rule,

$$
D_{3}(\zeta)=4 \delta(\zeta-A(u)) \delta(\zeta-A(v)) \nabla A(u) \cdot \nabla A(v) .
$$

Combining these formal computations we finally arrive at (1.13):

$$
\int_{\mathbb{R}} D(\zeta) \mathrm{d} \zeta=-2 \int_{\mathbb{R}} \delta(\zeta-A(u)) \delta(\zeta-A(v))|\nabla A(u)-\nabla A(v)|^{2} \mathrm{~d} \zeta \leq 0 .
$$

One crucial insight in [25] is that the convergence rate can be improved if one can send a certain parameter $\varepsilon$ to zero independently of the grid size $\Delta x$, where $\varepsilon$ controls the regularization of the Kružkov entropies. In this paper the regularization of the entropies is replaced by the regularization of the $\chi$ functions, and as before we would like to send $\varepsilon$ to zero independently of $\Delta x$ (and other parameters). It turns out that in one spatial dimension we can do this, reaching the convergence rate $\gamma=1 / 3$ as in [25]. In several dimensions we have not been able to carry out this " $\varepsilon \rightarrow 0$ before other parameters" program.

A serious difficulty stems from the lack of a chain rule for finite differences, in combination with the highly nonlinear nature of the dissipation function $D(\cdot)$, resulting in a series of intricate error terms. A feature of the kinetic approach is that the crucial error term can be expressed via the parabolic dissipation measure. To be a bit more precise, at the continuous level, the convergence rate $\gamma=1 / 3$ in the one-dimensional case depends decisively on the (weak) continuity of the map

$$
c \mapsto \int_{\mathbb{R}} \delta(\zeta-c) m_{A(u)}(\zeta) \mathrm{d} \zeta=\delta(c-A(u))\left(\partial_{x} A(u)\right)^{2},
$$

where $u$ is the entropy solution and $m_{A(u)}$ is the parabolic dissipation measure. The continuity of this map follows from (1.7). Unfortunately, in several space dimensions the continuity becomes a subtle matter, since the parabolic dissipation measure splits into directional components,

$$
m_{A(u)}=\sum_{i=1}^{d} m_{A(u)}^{i}, \quad m_{A(u)}^{i}=\delta(\zeta-A(u))\left(\partial_{x_{i}} A(u)\right)^{2} .
$$

It appears difficult to claim from the kinetic equation (1.7) the continuity of the individual components

$$
c \mapsto \int_{\mathbb{R}} \delta(\zeta-c) m_{A(u)}^{i}(\zeta) \mathrm{d} \zeta=\delta(c-A(u))\left(\partial_{x_{i}} A(u)\right)^{2}, \quad i=1, \ldots, d .
$$

Not being able to send the $\chi$-regularisation parameter $\varepsilon$ to zero, we must instead balance $\varepsilon$ against the grid size $\Delta x$ and a number of other parameters, at long last arriving at (1.3) with the convergence rate (1.6).

The optimality of $(1.6)(d>1)$, in the $L^{\infty} \cap B V$ class, is an open problem. It is informative to compare with recent results on viscosity solutions and error bounds for degenerate fully nonlinear elliptic and parabolic equations. We refer to Krylov [27], Barles and Jakobsen [4], and Caffarelli and Souganidis [7] for some recent works. For monotone approximations of fully nonlinear, first-order equations with Lipschitz solutions, Crandall and Lions [15] proved in 1984 the optimal $L^{\infty}$ convergence rate $1 / 2$. However, finding a rate for degenerate second order equations remained an open problem. The first result is due to Krylov with the rate 1/27. Later Barles and Jakobsen improved this to to $1 / 5$, with a further improvent by Krylov to $1 / 2$ for equations with special structure. We remark that these results concern equations with convex nonlinearities. Caffarelli and Souganidis proved that there is an algebraic rate of convergence for a class of nonconvex equations. The convergence rate is not explicit but known to be some (small) positive number. Here we should point out that in our framework convexity plays no role; the error estimate applies to general nonlinearities. 
The remaining part of this paper is organized as follows: In Section 2 we gather some relevant a priori estimates for nondegenerate convection-diffusion equations and state precisely the definition of an entropy solution. The difference method and the main result are presented in Section 3. In Section 4 we supply certain kinetic formulations of the convection-diffusion and difference equations. Section 5 is devoted to the proof of the main result, achieved through the derivation of an error equation based on the kinetic formulations, along with a lengthy series of estimates bounding "unwanted" terms in this equation. In Appendix 5.4 we collect results relating to well-posedness and a priori estimates for the difference method.

\section{Viscosity APPROXimations And ENTROPy SOLUtions}

Let us define the viscosity approximations. Set $A^{\eta}(u):=A(u)+\eta u$ for any fixed $\eta>0$, and consider the the uniformly parabolic problem

$$
\begin{cases}u_{t}^{\eta}+\nabla \cdot f\left(u^{\eta}\right)=\Delta A^{\eta}\left(u^{\eta}\right), & (t, x) \in \Pi_{T}, \\ u^{\eta}(0, x)=u_{0}(x), & x \in \mathbb{R}^{d} .\end{cases}
$$

It is well-known that (2.1) admits a unique classical (smooth) solution. We collect some relevant (standard) estimates from [35].

Lemma 2.1. Suppose $u_{0} \in L^{\infty}\left(\mathbb{R}^{d}\right) \cap L^{1}\left(\mathbb{R}^{d}\right) \cap B V\left(\mathbb{R}^{d}\right)$, and let $u^{\eta}$ be the unique classical solution of $(2.1)$. Then for any $t>0$,

$$
\begin{aligned}
\left\|u^{\eta}(t, \cdot)\right\|_{L^{1}\left(\mathbb{R}^{d}\right)} & \leq\left\|u_{0}\right\|_{L^{1}\left(\mathbb{R}^{d}\right)}, \\
\left\|u^{\eta}(t, \cdot)\right\|_{L^{\infty}\left(\mathbb{R}^{d}\right)} & \leq\left\|u_{0}\right\|_{L^{\infty}\left(\mathbb{R}^{d}\right)}, \\
\left|u^{\eta}(t, \cdot)\right|_{B V\left(\mathbb{R}^{d}\right)} & \leq\left|u_{0}\right|_{B V\left(\mathbb{R}^{d}\right)} .
\end{aligned}
$$

Lemma 2.2. Suppose $u_{0} \in L^{\infty}\left(\mathbb{R}^{d}\right) \cap L^{1}\left(\mathbb{R}^{d}\right)$ and $\nabla \cdot\left(f\left(u_{0}\right)-\nabla A\left(u_{0}\right)\right) \in L^{1}\left(\mathbb{R}^{d}\right)$. Let $u^{\eta}$ be the unique classical solution of (2.1). Then for any $t_{1}, t_{2}>0$,

$$
\left\|u^{\eta}\left(t_{2}, \cdot\right)-u^{\eta}\left(t_{1}, \cdot\right)\right\|_{L^{1}(\mathbb{R})} \leq\left\|\nabla \cdot\left(f\left(u_{0}\right)-\nabla A\left(u_{0}\right)\right)\right\|_{L^{1}\left(\mathbb{R}^{d}\right)}\left|t_{2}-t_{1}\right| .
$$

These results imply that the family $\left\{u^{\eta}\right\}_{\eta>0}$ is relatively compact in $C\left([0, T] ; L_{l o c}^{1}\left(\mathbb{R}^{d}\right)\right)$. If $u=\lim _{\eta \rightarrow 0} u^{\eta}$, then

$$
\left\|u^{\eta}-u\right\|_{L^{1}\left(\Pi_{T}\right)} \leq C \eta^{1 / 2},
$$

for some constant $C$ which does not depend on $\eta$, see, e.g., [19]. Moreover, $u$ is an entropy solution according to the following definition.

Definition 2.3. An entropy solution of (1.1) is a measurable function $u=u(t, x)$ satisfying:

(D.1) $u \in L^{\infty}\left([0, T] ; L^{1}\left(\mathbb{R}^{d}\right)\right) \cap L^{\infty}\left(\Pi_{T}\right) \cap C\left((0, T) ; L^{1}\left(\mathbb{R}^{d}\right)\right)$.

(D.2) $A(u) \in L^{2}\left([0, T] ; H^{1}\left(\mathbb{R}^{d}\right)\right)$.

(D.3) For all constants $c \in \mathbb{R}$ and all test functions $0 \leq \phi \in C_{0}^{\infty}\left(\mathbb{R}^{d} \times[0, T)\right)$, the following entropy inequality holds:

$$
\iint_{\Pi_{T}}|u-c| \partial_{t} \phi+\operatorname{sign}(u-c)(f(u)-f(c)) \cdot \nabla \varphi+|A(u)-A(c)| \Delta \varphi \mathrm{d} t \mathrm{~d} x+\int_{\mathbb{R}^{d}}\left|u_{0}-c\right| \varphi(x, 0) \mathrm{d} x \geq 0 .
$$

The uniqueness of entropy solutions is proved in [8], see the introduction for additional references. 


\section{Difference method And MAin RESUlt}

Let $f=\left(f^{1}, \ldots, f^{d}\right)$, and let $\Delta x$ denote the mesh size. For simplicity we consider a uniform grid in $\mathbb{R}^{d}$ consisting of cubes with sides $\Delta x$. For a multi-index $\alpha=\left(\alpha_{1}, \ldots, \alpha_{d}\right) \in \mathbb{Z}^{d}$, we let $I_{\alpha}$ denote the grid cell

$$
I_{\alpha}=\left[x_{\alpha_{1}-1 / 2}, x_{\alpha_{1}+1 / 2}\right) \times \cdots \times\left[x_{\alpha_{d}-1 / 2}, x_{\alpha_{d}+1 / 2}\right),
$$

where $x_{j+1 / 2}=(j+1 / 2) \Delta x$ for $j \in \mathbb{Z}$. Let $e_{k} \in \mathbb{Z}^{d}$ be the vector with value one in the $k$ th component and zero otherwise. Then we define the forward and backward discrete partial derivatives in the $k$ th direction as

$$
D_{ \pm}^{k}\left(\sigma_{\alpha}\right)= \pm \frac{\sigma_{\alpha \pm e_{k}}-\sigma_{\alpha}}{\Delta x} \quad k=1, \ldots, d .
$$

Definition 3.1 (numerical flux). We call a function $F \in C^{1}\left(\mathbb{R}^{2}\right)$ a monotone two point numerical flux for $f$, if $F(u, u)=f(u)$ and

$$
\frac{\partial}{\partial u} F(u, v) \geq 0 \quad \text { and } \quad \frac{\partial}{\partial v} F(u, v) \leq 0
$$

holds for all $u$ and $v$. We say that the numerical flux splits whenever $F$ can be written

$$
F(u, v)=F_{1}(u)+F_{2}(v) .
$$

Note that $F_{1}^{\prime} \geq 0$ and $F_{2}^{\prime} \leq 0$ whenever $F$ is monotone.

Let $F^{k}$ be a numerical flux function corresponding to $f^{k}$ for $k=1, \ldots, d$. The semi-discrete approximation of (1.1) is the solution of the equations

$$
\begin{cases}\frac{\mathrm{d}}{\mathrm{d} t} u_{\alpha}+\sum_{i=1}^{d} D_{-}^{i} F^{i}\left(u_{\alpha}, u_{\alpha+e_{i}}\right)=\sum_{i=1}^{d} D_{-}^{i} D_{+}^{i} A\left(u_{\alpha}\right), & \alpha \in \mathbb{Z}^{d}, t \in(0, T), \\ u_{\alpha}(0)=u_{\alpha, 0}, & \alpha \in \mathbb{Z}^{d},\end{cases}
$$

where $u_{\alpha, 0}=\frac{1}{\Delta x^{d}} \int_{I_{\alpha}} u_{0}(x) \mathrm{d} x$. See Appendix 5.4, in particular Lemmas A.2 and A.3, regarding existence and solution properties to this infinite system of ODEs.

Define the piecewise constant (in $x$ ) function $u_{\Delta x}$ by

$$
u_{\Delta x}(t, x)=u_{\alpha}(t) \text { for } x \in I_{\alpha} .
$$

Our main result is the following:

Theorem 3.2. Suppose $f$ and $A$ satisfy (1.2) and the initial function $u_{0}$ is in $B V\left(\mathbb{R}^{d}\right) \cap L^{\infty}\left(\mathbb{R}^{d}\right) \cap L^{1}\left(\mathbb{R}^{d}\right)$. Let $F^{i}$ be a monotone, Lipschitz, two point numerical flux corresponding to $f^{i}$ that splits for $1 \leq i \leq d$. Let $u$ be the entropy solution to (1.1) and $u_{\Delta x}$ be defined by (3.2), where $u_{\alpha}$ is the solution to (3.1).

Then, for any positive $R$ and $T$, there exists a constant $C$ depending only on $f, A, u_{0}, R$ and $T$, such that

$$
\left\|u_{\Delta x}(t)-u(t)\right\|_{L^{1}(B(0, R))} \leq C \Delta x^{\frac{2}{19+d}}, \quad t \in[0, T] .
$$

\section{Kinetic Formulations}

In this section we supply certain kinetic formulations of the continuous and discrete equations (1.1) and (3.1). As a preparation for the error estimate, we also regularize the kinetic equations by mollification. As explained in the introduction, due to the application of the viscous approximations in the proof of the error estimate, we assume $A^{\prime}>0$ for these intermediate results. 


\subsection{Kinetic formulation of convection-diffusion equation}

Lemma 4.1. Assume that $A^{\prime}>0$ and set $B:=A^{-1}$. Let $u$ be the solution of $(1.1)$. Define $g$ by $g(A(z))=f(z)$ for all $z \in \mathbb{R}$. Let $S \in C^{2}(\mathbb{R})$,

$$
\begin{array}{ll}
\psi(u)=\int_{0}^{u} S^{\prime}(z) B^{\prime}(z) \mathrm{d} z, & \psi_{A}(u)=\psi(A(u)), \\
q(u)=\int_{0}^{u} S^{\prime}(z) g^{\prime}(z) \mathrm{d} z, & q_{A}(u)=q(A(u)),
\end{array}
$$

and $S_{A}(u)=S(A(u))$. Then

$$
\partial_{t} \psi_{A}(u)+\nabla \cdot q_{A}(u)-\Delta S_{A}(u)=-S_{A}^{\prime \prime}(u)|\nabla A(u)|^{2} .
$$

Proof. Multiplying (1.1) by $\psi_{A}^{\prime}(u)$ gives

$$
\partial_{t} \psi_{A}(u)+\psi_{A}^{\prime}(u) \nabla \cdot f(u)=\psi_{A}^{\prime}(u) \Delta A(u) .
$$

Using a change of variables $A(\sigma)=z$,

$$
\begin{aligned}
q_{A}^{\prime}(u) & =\partial_{u}\left(\int_{0}^{A(u)} S^{\prime}(z) g^{\prime}(z) \mathrm{d} z\right) \\
& =\partial_{u}\left(\int_{0}^{u} S^{\prime}(A(\sigma)) g^{\prime}(A(\sigma)) A^{\prime}(\sigma) \mathrm{d} \sigma\right)=S^{\prime}(A(u)) f^{\prime}(u)
\end{aligned}
$$

Hence

$$
\psi_{A}^{\prime}(u) \nabla \cdot f(u)=\nabla \cdot q_{A}(u)
$$

Similarly we obtain $\psi_{A}^{\prime}(u)=S^{\prime}(A(u))$. Finally, observe that

$$
\Delta S_{A}(u)=S^{\prime \prime}(A(u))|\nabla A(u)|^{2}+\psi_{A}^{\prime}(u) \Delta A(u)
$$

The above entropy equation can be rephrased in terms of the $\chi$ function. Recall that for any locally Lipschitz continuous $\Psi: \mathbb{R} \rightarrow \mathbb{R}$,

$$
\Psi(u)-\Psi(0)=\int_{\mathbb{R}} \Psi^{\prime}(\xi) \chi(u ; \xi) \mathrm{d} \xi, \quad(u \in \mathbb{R}) .
$$

The next lemma reveals the equation satisfied by $\chi(A(u) ; \zeta)$, where $u$ solves $(1.1)$, i.e., the kinetic formulation of the convection-diffusion equation.

Lemma 4.2. Assume that $A^{\prime}>0$ and set $B:=A^{-1}$. Let $u$ be the solution of (1.1). Define $\rho(t, x, \zeta)=$ $\chi(A(u(t, x)) ; \zeta)$. Then

$$
\begin{cases}B^{\prime}(\zeta) \partial_{t} \rho+g^{\prime}(\zeta) \cdot \nabla \rho-\Delta \rho=\partial_{\zeta} m & \text { in } \mathcal{D}^{\prime}\left((0, T) \times \mathbb{R}^{d} \times \mathbb{R}\right) \\ \rho(0, x, \zeta)=\chi\left(A\left(u_{0}(x)\right) ; \zeta\right), & (x, \zeta) \in \mathbb{R}^{d} \times \mathbb{R}\end{cases}
$$

where

$$
m(t, x, \zeta)=\delta(\zeta-A(u))|\nabla A(u)|^{2},
$$

and $g$ satisfies $g(A(z))=f(z)$ for all $z \in \mathbb{R}$.

Proof. By Lemmas 4.1 and (4.1),

$\partial_{t} \int_{\mathbb{R}} S^{\prime}(\zeta) B^{\prime}(\zeta) \chi(A(u) ; \zeta) \mathrm{d} \zeta+\nabla \cdot \int_{\mathbb{R}} S^{\prime}(\zeta) g^{\prime}(\zeta) \chi(A(u) ; \zeta) \mathrm{d} \zeta-\Delta \int_{\mathbb{R}} S^{\prime}(\zeta) \chi(A(u) ; \zeta) \mathrm{d} \zeta=\int_{\mathbb{R}} S^{\prime}(\zeta) \partial_{\zeta} m(t, x, \zeta) \mathrm{d} \zeta$. 


\subsection{Kinetic formulation of discrete equations}

Stability/uniqueness analysis for differential equations often revolve around the chain rule. The chain rule breaks down for numerical methods, but for us the next lemma will act as a substitute.

Lemma 4.3. Let $S \in C^{2}(\mathbb{R})$ satisfy $S^{\prime}(0)=0$. For any $g \in C^{1}(\mathbb{R})$ and any real numbers $a, b$ and $c$,

$$
S^{\prime}(a)(g(b)-g(a))=\int_{0}^{b} S^{\prime}(z) g^{\prime}(z) \mathrm{d} z-\int_{0}^{a} S^{\prime}(z) g^{\prime}(z) \mathrm{d} z+\int_{a}^{b} S^{\prime \prime}(z)(g(z)-g(b)) \mathrm{d} z .
$$

Proof. For any $\zeta \in \mathbb{R}$, integration by parts yields

$$
S^{\prime}(\zeta)(g(\zeta)-g(b))=\int_{0}^{\zeta} S^{\prime}(z) g^{\prime}(z) \mathrm{d} z+\int_{0}^{\zeta} S^{\prime \prime}(z)(g(z)-g(b)) \mathrm{d} z .
$$

Take the two equations obtained by setting $\zeta$ be equal to $a$ and $b$ and subtract one from the other.

To make the discrete and continuous calculus notations similar, we introduce the discrete gradient

$$
D_{ \pm} \sigma=\left(D_{ \pm}^{1} \sigma, \ldots, D_{ \pm}^{d} \sigma\right), \quad \text { for any } \sigma: \mathbb{Z}^{d} \rightarrow \mathbb{R} .
$$

The upcoming lemma contains the equation satisfied by $\chi\left(u_{\alpha} ; \zeta\right)$, where $u_{\alpha}$ is the solution of the scheme (3.1).

Lemma 4.4. Suppose $A^{\prime} \geq 0$. Let $\left\{u_{\alpha}\right\}_{\alpha \in \mathbb{Z}^{d}}$ be the solution to (3.1). Then $\rho_{\alpha}(t, \xi):=\chi\left(u_{\alpha}(t) ; \xi\right)$ satisfies

$$
\begin{aligned}
& \partial_{t} \rho+\left(F_{1}^{\prime}(\xi) \cdot D_{-}+F_{2}^{\prime}(\xi) \cdot D_{+}\right) \rho-A^{\prime}(\xi) D_{-} \cdot D_{+} \rho=\partial_{\xi}\left(m_{F}+m_{A}\right), \\
& \zeta_{\alpha}(0, \xi)=\chi\left(u_{\alpha, 0} ; \xi\right),
\end{aligned}
$$

in $\mathcal{D}^{\prime}(\mathbb{R} \times[0, T])$ for each $\alpha \in \mathbb{Z}^{d}$, where

$$
m_{F}=\sum_{i=1}^{d}\left(\left(F_{1}^{i}(\xi)-F_{1}^{i}\left(u_{\alpha-e_{i}}\right)\right) D_{-}^{i} \chi\left(u_{\alpha} ; \xi\right)+\left(F_{2}^{i}(\xi)-F_{2}^{i}\left(u_{\alpha+e_{i}}\right)\right) D_{+}^{i} \chi\left(u_{\alpha} ; \xi\right)\right)
$$

and

$$
m_{A}=\sum_{i=1}^{d}\left(\frac{1}{\Delta x}\left(A\left(u_{\alpha+e_{i}}\right)-A(\xi)\right) D_{+}^{i} \chi\left(u_{\alpha} ; \xi\right)+\frac{1}{\Delta x}\left(A(\xi)-A\left(u_{\alpha-e_{i}}\right)\right) D_{-}^{i} \chi\left(u_{\alpha} ; \xi\right)\right) .
$$

Proof. Since $\left\{u_{\alpha}\right\}$ is a solution of (3.1),

$$
S^{\prime}\left(u_{\alpha}(t)\right) \partial_{t} u_{\alpha}(t)+\sum_{i=1}^{d} S^{\prime}\left(u_{\alpha}(t)\right) D_{-}^{i} F^{i}\left(u_{\alpha}(t), u_{\alpha+e_{i}}(t)\right)=\sum_{i=1}^{d} S^{\prime}\left(u_{\alpha}(t)\right) D_{-}^{i} D_{+}^{i} A\left(u_{\alpha}(t)\right),
$$

for all $t \in(0, T)$ and $\alpha \in \mathbb{Z}^{d}$. By the chain rule

$$
S^{\prime}\left(u_{\alpha}(t)\right) \partial_{t} u_{\alpha}(t)=\partial_{t} S\left(u_{\alpha}(t)\right) .
$$

Consider the flux term. For each $i$, we have that $F^{i}=F_{2}^{i}+F_{2}^{i}$, and therefore

$$
S^{\prime}\left(u_{\alpha}(t)\right) D_{-}^{i} F^{i}\left(u_{\alpha}(t), u_{\alpha+e_{i}}(t)\right)=S^{\prime}\left(u_{\alpha}(t)\right) D_{-}^{i} F_{1}^{i}\left(u_{\alpha}\right)+S^{\prime}\left(u_{\alpha}(t)\right) D_{+}^{i} F_{2}^{i}\left(u_{\alpha}\right) .
$$


By Lemma 4.3, with $g$ equal to $F_{1}^{i}$ and $F_{2}^{i}$, we obtain

$$
\begin{aligned}
& S^{\prime}\left(u_{\alpha}(t)\right) D_{-}^{i} F_{1}^{i}\left(u_{\alpha}\right)=D_{-}^{i} Q_{1}^{i}\left(u_{\alpha}\right)-\frac{1}{\Delta x} \int_{u_{\alpha}}^{u_{\alpha-e_{i}}} S^{\prime \prime}(z)\left(F_{1}^{i}(z)-F_{1}^{i}\left(u_{\alpha-e_{i}}\right)\right) \mathrm{d} z, \\
& S^{\prime}\left(u_{\alpha}(t)\right) D_{+}^{i} F_{2}^{i}\left(u_{j}\right)=D_{+}^{i} Q_{2}^{i}\left(u_{\alpha}\right)+\frac{1}{\Delta x} \int_{u_{\alpha}}^{u_{\alpha+e_{i}}} S^{\prime \prime}(z)\left(F_{2}^{i}(z)-F_{2}^{i}\left(u_{\alpha+e_{i}}\right)\right) \mathrm{d} z
\end{aligned}
$$

where

$$
Q_{j}^{i}(u):=\int_{0}^{u} S^{\prime}(z)\left(F_{j}^{i}\right)^{\prime}(z) \mathrm{d} z \text { for } j=1,2 .
$$

Consider the term on the right-hand side of (4.3). Let

$$
R(u):=\int_{0}^{u} S^{\prime}(z) A^{\prime}(z) \mathrm{d} z
$$

Fix $i$ and apply Lemma 4.3 with $g=A, a=u_{\alpha}, b=u_{\alpha-e_{i}}$, and $u_{\alpha+e_{i}}$. Adding the two equations yields

$$
\begin{aligned}
S^{\prime}\left(u_{\alpha}\right) D_{-}^{i} D_{+}^{i}\left(A\left(u_{\alpha}\right)\right)= & D_{-}^{i} D_{+}^{i} R\left(u_{\alpha}\right) \\
& +\frac{1}{\Delta x^{2}} \int_{u_{\alpha}}^{u_{\alpha+e_{i}}} S^{\prime \prime}(z)\left(A(z)-A\left(u_{\alpha+e_{i}}\right)\right) \mathrm{d} z \\
& +\frac{1}{\Delta x^{2}} \int_{u_{\alpha}}^{u_{\alpha-e_{i}}} S^{\prime \prime}(z)\left(A(z)-A\left(u_{\alpha-e_{i}}\right)\right) \mathrm{d} z .
\end{aligned}
$$

Hence (4.3) turns into

$$
\begin{aligned}
\partial_{t} S\left(u_{\alpha}\right)+\sum_{i=1}^{d}\left(D_{-}^{i} Q_{1}^{i}\left(u_{\alpha}\right)+\right. & \left.D_{+}^{i} Q_{2}^{i}\left(u_{\alpha}\right)\right)-\sum_{i=1}^{d} D_{-}^{i} D_{+}^{i} R\left(u_{\alpha}\right) \\
= & \sum_{i=1}^{d} \frac{1}{\Delta x} \int_{u_{\alpha}}^{u_{\alpha-e_{i}}} S^{\prime \prime}(z)\left(F_{1}^{i}(z)-F_{1}^{i}\left(u_{\alpha-e_{i}}\right)\right) \mathrm{d} z \\
& -\sum_{i=1}^{d} \frac{1}{\Delta x} \int_{u_{\alpha}}^{u_{\alpha+e_{i}}} S^{\prime \prime}(z)\left(F_{2}^{i}(z)-F_{2}^{i}\left(u_{\alpha+e_{i}}\right)\right) \mathrm{d} z \\
& +\sum_{i=1}^{d} \frac{1}{\Delta x^{2}} \int_{u_{\alpha}}^{u_{\alpha+e_{i}}} S^{\prime \prime}(z)\left(A(z)-A\left(u_{\alpha+e_{i}}\right)\right) \mathrm{d} z \\
& +\sum_{i=1}^{d} \frac{1}{\Delta x^{2}} \int_{u_{\alpha}}^{u_{\alpha-e_{i}}} S^{\prime \prime}(z)\left(A(z)-A\left(u_{\alpha-e_{i}}\right)\right) \mathrm{d} z .
\end{aligned}
$$

By equation (4.1),

$$
D_{-}^{i} Q_{1}^{i}\left(u_{\alpha}\right)+D_{+}^{i} Q_{2}^{i}\left(u_{\alpha}\right)=\int_{\mathbb{R}} S^{\prime}(\xi)\left(\left(F_{1}^{i}\right)^{\prime}(\xi) D_{-}^{i}+\left(F_{2}^{i}\right)^{\prime}(\xi) D_{+}^{i}\right) \chi\left(u_{\alpha} ; \xi\right) \mathrm{d} \xi
$$

Similarly,

$$
D_{-}^{i} D_{+}^{i} R\left(u_{\alpha}\right)=\int_{\mathbb{R}} S^{\prime}(\xi) A^{\prime}(\xi) D_{-}^{i} D_{+}^{i} \chi\left(u_{\alpha} ; \xi\right) \mathrm{d} \xi .
$$

Consider the right-hand side. For any $g \in C(\mathbb{R})$,

$$
\int_{a}^{b} S^{\prime \prime}(z)(g(z)-g(b)) \mathrm{d} z=\int_{\mathbb{R}} S^{\prime \prime}(\xi)(g(\xi)-g(b))(\chi(b ; \xi)-\chi(a ; \xi)) \mathrm{d} \xi .
$$


Hence

$$
\begin{aligned}
& \frac{1}{\Delta x} \int_{u_{\alpha}}^{u_{\alpha-e_{i}}}\left(F_{1}^{i}(z)-F_{1}^{i}\left(u_{\alpha-e_{i}}\right) \mathrm{d} z=-\int_{\mathbb{R}} S^{\prime \prime}(\xi)\left(F_{1}^{i}(\xi)-F_{1}^{i}\left(u_{\alpha-e_{i}}\right)\right) D_{-}^{i} \chi\left(u_{\alpha} ; \xi\right) \mathrm{d} \xi\right. \\
& \frac{-1}{\Delta x} \int_{u_{\alpha}}^{u_{\alpha+e_{i}}}\left(F_{2}^{i}(z)-F_{2}^{i}\left(u_{\alpha+e_{i}}\right) \mathrm{d} z=-\int_{\mathbb{R}} S^{\prime \prime}(\xi)\left(F_{2}^{i}(\xi)-F_{2}^{i}\left(u_{\alpha+e_{i}}\right)\right) D_{+}^{i} \chi\left(u_{\alpha} ; \xi\right) \mathrm{d} \xi .\right.
\end{aligned}
$$

Similarly,

$$
\begin{aligned}
\frac{1}{\Delta x^{2}} \int_{u_{\alpha}}^{u_{\alpha+e_{i}}} S^{\prime \prime}(z)( & \left.A(z)-A\left(u_{\alpha+e_{i}}\right)\right) \mathrm{d} z \\
= & \frac{-1}{\Delta x} \int_{\mathbb{R}} S^{\prime \prime}(\xi)\left(A\left(u_{\alpha+e_{i}}\right)-A(\xi)\right) D_{+}^{i} \chi\left(u_{\alpha} ; \xi\right) \mathrm{d} \xi \\
\frac{1}{\Delta x^{2}} \int_{u_{\alpha}}^{u_{\alpha-e_{i}}} S^{\prime \prime}(z)( & \left(A(z)-A\left(u_{\alpha-e_{i}}\right)\right) \mathrm{d} z \\
= & \frac{-1}{\Delta x} \int_{\mathbb{R}} S^{\prime \prime}(\xi)\left(A(\xi)-A\left(u_{\alpha-e_{i}}\right)\right) D_{-}^{i} \chi\left(u_{\alpha} ; \xi\right) \mathrm{d} \xi
\end{aligned}
$$

The result follows.

For a function $u: \mathbb{R}^{d} \rightarrow \mathbb{R}$ we define the shift operator $S_{y}$ by $S_{y} u(x)=u(x+y)$. Then the discrete derivatives may be expressed as

$$
D_{ \pm}^{i} u= \pm \frac{S_{ \pm \Delta x_{i}} u-u}{\Delta x},
$$

where $\Delta x_{i}=\Delta x e_{i}$.

Making a change of variable $\zeta=A(\xi)$, we can obtain an equation satisfied by $\chi\left(A\left(u_{\Delta x}\right) ; \zeta\right)$, where $u_{\Delta x}$ is the numerical solution (3.2), resulting in the "discrete" kinetic formulation to be utilized later.

Lemma 4.5. Suppose $A^{\prime}>0$. Let $\left\{u_{\alpha}\right\}$ be the solution to (3.1) and define $u_{\Delta x}$ by (3.2). Let $G_{j}: \mathbb{R}_{\rightarrow} \rightarrow \mathbb{R}^{d}$ satisfy $G_{j}(A(u))=F_{j}(u) \forall u$, for $j=1,2$. Then $\rho^{\Delta x}(t, x, \zeta)=\chi\left(A\left(u_{\Delta x}(t, x)\right) ; \zeta\right)$ satisfies

$$
\begin{aligned}
& B^{\prime}(\zeta) \partial_{t} \rho^{\Delta x}+\left(G_{1}^{\prime}(\zeta) \cdot D_{+}+G_{2}^{\prime}(\zeta) \cdot D_{-}\right) \rho^{\Delta x}-D_{-} \cdot D_{+} \rho^{\Delta x}=\partial_{\zeta}\left(n_{A}^{\Delta x}+n_{G}^{\Delta x}\right), \\
& \rho^{\Delta x}(0, \zeta)=\chi\left(A\left(u_{\Delta x}^{0}\right) ; \zeta\right),
\end{aligned}
$$

in $\mathcal{D}^{\prime}\left(\mathbb{R} \times \Pi_{T}\right)$, where

$$
n_{G}^{\Delta x}=\sum_{i=1}^{d}\left(G_{1}^{i}(\zeta)-G_{1}^{i}\left(A\left(S_{-\Delta x_{i}} u_{\Delta x}\right)\right)\right) D_{-}^{i} \chi\left(A\left(u_{\Delta x}\right) ; \zeta\right)+\sum_{i=1}^{d}\left(G_{2}^{i}(\zeta)-G_{2}^{i}\left(A\left(S_{\Delta x_{i}} u_{\Delta x}\right)\right)\right) D_{+}^{i} \chi\left(A\left(u_{\Delta x}\right) ; \zeta\right)
$$

and

$$
n_{A}^{\Delta x}=\sum_{i=1}^{d} \frac{1}{\Delta x}\left(A\left(S_{\Delta x_{i}} u_{\Delta x}\right)-\zeta\right) D_{+}^{i} \chi\left(A\left(u_{\Delta x}\right) ; \zeta\right)+\sum_{i=1}^{d} \frac{1}{\Delta x}\left(\zeta-A\left(S_{-\Delta x_{i}} u_{\Delta x}\right)\right) D_{-}^{i} \chi\left(A\left(u_{\Delta x}\right) ; \zeta\right) .
$$

Proof. Let $S \in C_{c}^{\infty}(\mathbb{R})$ and define $S_{A}(\xi)=S(A(\xi))$. By Lemma 4.4,

$$
\begin{aligned}
& \partial_{t} \int_{\mathbb{R}} S_{A}(\xi) \chi\left(u_{\alpha} ; \xi\right) \mathrm{d} \xi+\int_{\mathbb{R}} S_{A}(\xi)\left(F_{1}^{\prime}(\xi) \cdot D_{-}+F_{2}^{\prime}(\xi) \cdot D_{+}\right) \chi\left(u_{\alpha} ; \xi\right) \mathrm{d} \xi \\
& -\int_{\mathbb{R}} S_{A}(\xi) A^{\prime}(\xi) D_{-} \cdot D_{+} \chi\left(u_{\alpha} ; \xi\right) \mathrm{d} \xi=-\int_{\mathbb{R}} S_{A}^{\prime}(\xi)\left(m_{F}+m_{A}\right) \mathrm{d} \xi .
\end{aligned}
$$


Let $\zeta=A(\xi)$ and note that $\chi\left(u_{\alpha} ; \xi\right)=\chi\left(A\left(u_{\alpha}\right) ; A(\xi)\right)$. The terms on the left-hand side are straightforward to verify. Next,

$$
\begin{aligned}
\int_{\mathbb{R}} S_{A}^{\prime}(\xi) m_{F}(\xi) \mathrm{d} \xi= & \sum_{i=1}^{d} \int_{\mathbb{R}} S^{\prime}(A(\xi))\left(G_{1}^{i}(A(\xi))-G_{1}^{i}\left(A\left(u_{\alpha-e_{i}}\right)\right)\right) D_{-}^{i} \chi\left(A\left(u_{\alpha}\right) ; A(\xi)\right) A^{\prime}(\xi) \mathrm{d} \xi \\
& +\sum_{i=1}^{d} \int_{\mathbb{R}} S^{\prime}(A(\xi))\left(G_{2}^{i}(A(\xi))-G_{2}^{i}\left(A\left(u_{\alpha+e_{i}}\right)\right)\right) D_{+}^{i} \chi\left(A\left(u_{\alpha}\right) ; A(\xi)\right) A^{\prime}(\xi) \mathrm{d} \xi \\
= & \sum_{i=1}^{d} \int_{\mathbb{R}} S^{\prime}(\zeta)\left(G_{1}^{i}(\zeta)-G_{1}^{i}\left(A\left(u_{\alpha-e_{i}}\right)\right)\right) D_{-}^{i} \chi\left(A\left(u_{\alpha}\right) ; \zeta\right) \mathrm{d} \zeta \\
& +\sum_{i=1}^{d} \int_{\mathbb{R}} S^{\prime}(\zeta)\left(G_{2}^{i}(\zeta)-G_{2}^{i}\left(A\left(u_{\alpha+e_{i}}\right)\right)\right) D_{+}^{i} \chi\left(A\left(u_{\alpha}\right) ; \zeta\right) \mathrm{d} \zeta .
\end{aligned}
$$

A similar computation shows the second equality involving $n_{A}^{\Delta x}$.

\subsection{Various regularizations}

In this section we study mollified versions of Lemmas 4.2 and 4.5. Let us first introduce some notation. Let $J \in C_{c}^{\infty}(\mathbb{R})$ denote a function satisfying

$$
\operatorname{supp}(J) \subset[-1,1], \quad \int_{\mathbb{R}} J(x) \mathrm{d} x=1 \text { and } J(-x)=J(x)
$$

for all $x \in \mathbb{R}$. That is, $J$ is a symmetric mollifier on $\mathbb{R}$ with support in $[-1,1]$. For any $\sigma>0$ we let $J_{\sigma}(x)=$ $\sigma^{-1} J\left(\sigma^{-1} x\right)$. For any $n \geq 1, J_{\sigma}^{\otimes n}$ is a symmetric mollifier on $\mathbb{R}^{n}$ with support in $[-\sigma, \sigma]^{n}$. In general the dimension of the argument will define $n$, so to simplify the notation we write $J_{\sigma}$ instead of $J_{\sigma}^{\otimes n}$.

Let $\psi: \mathbb{R}^{2} \rightarrow \mathbb{R}$ be a continuous function and $u, v \in L^{1}(\mathbb{R})$. Then we define

$$
(\psi(u, v) \stackrel{(u, v)}{\star} f \otimes g)(x):=\int_{\mathbb{R}} \int_{\mathbb{R}} \psi\left(u\left(y_{1}\right), v\left(y_{2}\right)\right) f\left(x-y_{1}\right) g\left(x-y_{2}\right) \mathrm{d} y_{1} \mathrm{~d} y_{2},
$$

where $f, g \in L^{1}(\mathbb{R})$. Similarly, we let

$$
\psi(u, v) \stackrel{(u)}{\star} f:=\int_{\mathbb{R}} \psi(u(y), v(x)) f(x-y) \mathrm{d} y .
$$

This notation generalizes in an obvious way to functions of several variables.

We start by introducing regularizations of $\operatorname{sign}(\cdot)$ and $\chi(u ; \cdot)$

Lemma 4.6. For $\varepsilon>0$, define

$$
\operatorname{sign}_{\varepsilon}(\xi):=2 \int_{0}^{\xi} J_{\varepsilon}(\zeta) \mathrm{d} \zeta, \quad \chi_{\varepsilon}(u ; \xi):=\int_{\mathbb{R}} \chi(u ; \zeta) J_{\varepsilon}(\xi-\zeta) \mathrm{d} \zeta .
$$

Then

(i) For each $\xi, u \mapsto \chi_{\varepsilon}(u ; \xi) \in C^{\infty}(\mathbb{R})$ and $\partial_{u} \chi_{\varepsilon}(u ; \xi)=J_{\varepsilon}(\xi-u)$.

(ii) For all $u$ and $\xi$

$$
\operatorname{sign}_{\varepsilon}(\xi)-2 \chi_{\varepsilon}(u ; \xi)=\operatorname{sign}_{\varepsilon}(\xi-u) .
$$

(iii) For any $u$

$$
\int_{\mathbb{R}}\left|\chi_{\varepsilon}(u ; \xi)-\chi(u ; \xi)\right| \mathrm{d} \xi \leq 4 \varepsilon .
$$


Proof. We first prove (i). Let $H_{\varepsilon}^{\prime}(\sigma)=J_{\varepsilon}(\sigma)$. Since $J_{\varepsilon}(\xi-\zeta)=J_{\varepsilon}(\zeta-\xi)$,

$$
\lim _{h \rightarrow 0} \frac{1}{h} \int_{\mathbb{R}}(\chi(u+h ; \zeta)-\chi(u ; \zeta)) J_{\varepsilon}(\xi-\zeta) \mathrm{d} \zeta=\lim _{h \rightarrow 0} \frac{1}{h}\left(H_{\varepsilon}(u+h-\xi)-H_{\varepsilon}(u-\xi)\right)=J_{\varepsilon}(u-\xi) .
$$

Next we prove (ii). Let $\sigma=\zeta-\xi$. By the symmetry of $J_{\varepsilon}$,

$$
\chi_{\varepsilon}(u ; \xi)=\int_{\mathbb{R}} \chi(u ; \sigma+\xi) J_{\varepsilon}(\sigma) \mathrm{d} \sigma .
$$

A calculation (or (5.24)) yields

$$
\chi(u ; \sigma+\xi)=\chi(u-\xi ; \sigma)-\chi(-\xi ; \sigma) .
$$

Note that $\chi(-\xi ; \sigma)=-\chi(\xi ;-\sigma)$. Hence

$$
\chi_{\varepsilon}(u ; \xi)=\int_{\mathbb{R}}(\chi(u-\xi ; \zeta)+\chi(\xi ;-\zeta)) J_{\varepsilon}(\zeta) \mathrm{d} \zeta .
$$

It follows that

$$
\begin{aligned}
\operatorname{sign}_{\varepsilon}(\xi)-2 \chi_{\varepsilon}(u ; \xi) & =-2 \int_{\mathbb{R}} \chi(u-\xi ; \zeta) J_{\varepsilon}(\zeta) \mathrm{d} \zeta+2 \int_{\mathbb{R}}(\chi(\xi ; \zeta)-\chi(\xi ;-\zeta)) J_{\varepsilon}(\zeta) \mathrm{d} \zeta \\
& =: \mathscr{T}_{1}+\mathscr{T}_{2}
\end{aligned}
$$

Since $(\chi(\xi ; \zeta)-\chi(\xi ;-\zeta))$ is antisymmetric in $\zeta$ and $J_{\varepsilon}$ is symmetric it follows that $\mathscr{T}_{2}=0$. Now

$$
\mathscr{T}_{1}=-2 \int_{0}^{u-\xi} J_{\varepsilon}(\zeta) \mathrm{d} \zeta=2 \int_{0}^{\xi-u} J_{\varepsilon}(\zeta) \mathrm{d} \zeta=\operatorname{sign}_{\varepsilon}(\xi-u)
$$

To prove (iii), note that

$$
\left|\chi_{\varepsilon}(u ; \xi)-\chi(u ; \xi)\right|=0 \text { whenever } \xi \notin(-\varepsilon, \varepsilon) \cup(u-\varepsilon, u+\varepsilon) .
$$

For $\varepsilon>0$ and $f \in C(\mathbb{R})$, let $R_{\varepsilon}^{f}: \mathbb{R}^{2} \rightarrow \mathbb{R}$ be defined by

$$
\int_{\mathbb{R}} f(\sigma) \chi(u ; \sigma) J_{\varepsilon}(\zeta-\sigma) \mathrm{d} \sigma=R_{\varepsilon}^{f}(u, \zeta)+f(\zeta) \chi_{\varepsilon}(u ; \zeta),
$$

for all $u, \zeta \in \mathbb{R}$.

Now we are ready to provide "regularized" versions of Lemmas 4.2 and 4.5. As the mollification will take place on a slightly smaller region, we introduce the notation $\Pi_{T}^{r_{0}}:=\left(r_{0}, T-r_{0}\right) \times \mathbb{R}^{d}$.

We start with the regularization of the kinetic formulation of the convection-diffusion equation.

Lemma 4.7. Assume that $A^{\prime}>0$. Let $u$ be the solution of (1.1) and define

$$
\rho_{\varepsilon, r, r_{0}}:=\chi(A(u) ; \cdot) \star J_{r_{0}} \otimes J_{r} \otimes J_{\varepsilon} .
$$

Then for $(t, x, \zeta) \in \Pi_{T}^{r_{0}} \times \mathbb{R}$, the function $\rho_{\varepsilon, r, r_{0}}$ satisfies

$$
B^{\prime}(\zeta) \partial_{t} \rho_{\varepsilon, r, r_{0}}+g^{\prime}(\zeta) \cdot \nabla \rho_{\varepsilon, r, r_{0}}-\Delta \rho_{\varepsilon, r, r_{0}}+\partial_{t} R_{\varepsilon, r, r_{0}}^{B^{\prime}}+\nabla \cdot R_{\varepsilon, r, r_{0}}^{g^{\prime}}=\partial_{\zeta} n_{A, \varepsilon, r, r_{0}},
$$

where

$$
R_{\varepsilon, r, r_{0}}^{f}=R_{\varepsilon}^{f}(A(u), \zeta) \star J_{r} \otimes J_{r_{0}}
$$

with $R_{\varepsilon}^{f}$ defined by (4.5), and

$$
n_{A, \varepsilon, r, r_{0}}(t, x, \zeta)=\left(J_{\varepsilon}(\zeta-A(u))|\nabla A(u)|^{2} \star J_{r_{0}} \otimes J_{r}\right)(t, x) .
$$


Proof. Starting off from Lemma 4.2, take the convolution of equation (4.2) with $J_{\varepsilon}$ and apply (4.5). Finally, convolve the resulting equation with $J_{r} \otimes J_{r_{0}}$.

Next up is the regularization of the kinetic formulation of the discrete equations.

Lemma 4.8. Under the same assumptions and with the same notation as in Lemma 4.5, define

$$
\rho_{\varepsilon, r, r_{0}}^{\Delta x}:=\chi\left(A\left(u_{\Delta x}\right) ; \cdot\right) \star J_{r_{0}} \otimes J_{r} \otimes J_{\varepsilon} .
$$

For $(t, x, \zeta) \in \Pi_{T}^{r_{0}} \times \mathbb{R}$, the function $\rho_{\varepsilon, r, r_{0}}^{\Delta x}$ satisfies

$$
\begin{aligned}
B^{\prime}(\zeta) \partial_{t} \rho_{\varepsilon, r, r_{0}}^{\Delta x}+g^{\prime}(\zeta) \cdot \nabla & \rho_{\varepsilon, r, r_{0}}^{\Delta x}-\Delta \rho_{\varepsilon, r, r_{0}}^{\Delta x}+G_{1}^{\prime}(\zeta) \cdot\left(D_{+}-\nabla\right) \rho_{\varepsilon, r, r_{0}}^{\Delta x} \\
& +G_{2}^{\prime}(\zeta) \cdot\left(D_{-}-\nabla\right) \rho_{\varepsilon, r, r_{0}}^{\Delta x}+\left(\Delta-D_{-} \cdot D_{+}\right) \rho_{\varepsilon, r, r_{0}}^{\Delta x}+\partial_{t} R_{\varepsilon, r, r_{0}}^{B^{\prime}, \Delta x} \\
& \quad+D_{+} \cdot R_{\varepsilon, r, r_{0}}^{G_{1}^{\prime}, x_{0}}+D_{-} \cdot R_{\varepsilon, r, r_{0}}^{G_{2}^{\prime}, \Delta x}=\partial_{\zeta}\left(n_{A, \varepsilon, r, r_{0}}^{\Delta x}+n_{G, \varepsilon, r, r_{0}}^{\Delta x}\right)
\end{aligned}
$$

Here, $R_{\varepsilon, r, r_{0}}^{f, \Delta x}=R_{\varepsilon}^{f}\left(A\left(u_{\Delta x}\right), \cdot\right) \star J_{r} \otimes J_{r_{0}}$ with $R_{\varepsilon}^{f}$ coming from (4.5). Furthermore,

$$
n_{A, \varepsilon, r, r_{0}}^{\Delta x}=n_{A}^{\Delta x} \star\left(J_{\varepsilon} \otimes J_{r} \otimes J_{r_{0}}\right) \text { and } n_{G, \varepsilon, r, r_{0}}^{\Delta x}=n_{G}^{\Delta x} \star\left(J_{\varepsilon} \otimes J_{r} \otimes J_{r_{0}}\right) .
$$

Proof. In view of Lemmas 4.5 and (4.5),

$$
\begin{aligned}
B^{\prime}(\zeta) \partial_{t} \rho_{\varepsilon}^{\Delta x}+\left(G_{1}^{\prime}(\zeta) \cdot D_{+}+G_{2}^{\prime}(\zeta)\right. & \left.\cdot D_{-}\right) \rho_{\varepsilon}^{\Delta x}-D_{-} \cdot D_{+} \rho_{\varepsilon}^{\Delta x} \\
& +\partial_{t} R_{\varepsilon}^{B^{\prime}}\left(u_{\Delta x}, \zeta\right)+D_{+} \cdot R_{\varepsilon}^{G_{1}^{\prime}}\left(u_{\Delta x}, \zeta\right)+D_{-} \cdot R_{\varepsilon}^{G_{2}^{\prime}}\left(u_{\Delta x}, \zeta\right)=\partial_{\zeta}\left(n_{A, \varepsilon}^{\Delta x}+n_{G, \varepsilon}^{\Delta x}\right)
\end{aligned}
$$

where $\rho_{\varepsilon}^{\Delta x}(t, x, \zeta)=\chi_{\varepsilon}\left(A\left(u_{\Delta x}\right) ; \zeta\right)$ and $n_{A, \varepsilon}^{\Delta x}=n_{A}^{\Delta x} \star J_{\varepsilon}$ and $n_{G, \varepsilon}^{\Delta x}=n_{G}^{\Delta x} \star J_{\varepsilon}$. Take the convolution of the above equation with $J_{r} \otimes J_{r_{0}}$. Recall that $G_{1}^{\prime}+G_{2}^{\prime}=g^{\prime}$ and add and subtract to obtain the result.

\section{Proof of Theorem 3.2}

We are now ready to embark on the proof of the error estimate (Thm. 3.2). Instead of working directly with the microscopic contraction functional (1.8), we introduce a regularized version $Q_{\varepsilon}$ of it. For $u, v, \xi \in \mathbb{R}$, define

$$
Q_{\varepsilon}(u, v ; \xi):=\operatorname{sign}_{\varepsilon}(\xi) \chi_{\varepsilon}(u ; \xi)+\operatorname{sign}_{\varepsilon}(\xi) \chi_{\varepsilon}(v ; \xi)-2 \chi_{\varepsilon}(u ; \xi) \chi_{\varepsilon}(v ; \xi),
$$

where $\operatorname{sign}_{\varepsilon}$ and $\chi_{\varepsilon}$ are given in Lemma 4.6. One may show that

$$
\int_{\mathbb{R}}\left(\chi_{\varepsilon}(u ; \xi)-\chi_{\varepsilon}(v ; \xi)\right)^{2} \mathrm{~d} \xi=\int_{\mathbb{R}} Q_{\varepsilon}(u, v ; \xi) \mathrm{d} \xi
$$

This equality is, however, not directly useful to us, since we will be working with functions like $\chi_{\varepsilon}(A(u) ; \xi)$ with $A(\cdot)$ nonlinear, but see the related Lemma 5.18.

\subsection{Main error equation}

We will use the kinetic formulations of the convection-diffusion equation and the difference method to derive a fundamental equation for the error quantity $Q_{\varepsilon}\left(A(u(t, x)), A\left(u_{\Delta x}(t, x)\right) ; \zeta\right)$ (properly regularized).

Lemma 5.1. Assume that $A^{\prime}>0$. With the notation of Lemmas 4.7 and 4.8 , define

$$
Q_{\varepsilon, r, r_{0}}(\zeta)=Q_{\varepsilon}\left(A(u), A\left(u_{\Delta x}\right) ; \zeta\right) \stackrel{\left(u, u_{\Delta x}\right)}{\star}\left(J_{r_{0}} \otimes J_{r}\right) \otimes\left(J_{r_{0}} \otimes J_{r}\right) .
$$


Then, for all $(t, x) \in \Pi_{T}^{r_{0}}$,

$$
\begin{aligned}
\int_{\mathbb{R}} B^{\prime}(\zeta) \partial_{t} Q_{\varepsilon, r, r_{0}} \mathrm{~d} \zeta+\int_{\mathbb{R}} g^{\prime}(\zeta) \cdot \nabla Q_{\varepsilon, r, r_{0}} \mathrm{~d} \zeta \\
=\int_{\mathbb{R}} \Delta Q_{\varepsilon, r, r_{0}} \mathrm{~d} \zeta+2 \int_{\mathbb{R}} \nabla \rho_{\varepsilon, r, r_{0}} \cdot\left(2 \nabla-\left(D_{+}+D_{-}\right)\right) \rho_{\varepsilon, r, r_{0}}^{\Delta x} \mathrm{~d} \zeta \\
\quad-\int_{\mathbb{R}}\left(\operatorname{sign}_{\varepsilon}\left(\zeta-A\left(u_{\Delta x}\right)\right) \star J_{r_{0}} \otimes J_{r}\right) \partial_{t} R_{\varepsilon, r, r_{0}}^{B^{\prime}} \mathrm{d} \zeta \\
\quad-\int_{\mathbb{R}}\left(\operatorname{sign}_{\varepsilon}(\zeta-A(u)) \star J_{r_{0}} \otimes J_{r}\right) \partial_{t} R_{\varepsilon, r, r_{0}}^{B^{\prime}, \Delta x} \mathrm{~d} \zeta \\
\quad-\int_{\mathbb{R}}\left(\operatorname{sign}_{\varepsilon}\left(\zeta-A\left(u_{\Delta x}\right)\right) \star J_{r_{0}} \otimes J_{r}\right) \nabla \cdot R_{\varepsilon, r, r_{0}}^{g^{\prime}} \mathrm{d} \zeta \\
\quad-\int_{\mathbb{R}}\left(\operatorname{sign}_{\varepsilon}(\zeta-A(u)) \star J_{r_{0}} \otimes J_{r}\right)\left(D_{+} \cdot R_{\varepsilon, r, r_{0}}^{G_{1}^{\prime}, \Delta x}+D_{-} \cdot R_{\varepsilon, r, r_{0}}^{G_{2}^{\prime}, \Delta x}\right) \mathrm{d} \zeta \\
\quad-\int_{\mathbb{R}}\left(\operatorname{sign}_{\varepsilon}(\zeta-A(u)) \star J_{r_{0}} \otimes J_{r}\right)\left(G_{1}^{\prime}(\zeta) \cdot\left(D_{+}-\nabla\right)\right. \\
\left.\quad+G_{2}^{\prime}(\zeta) \cdot(D-\nabla)\right) \rho_{\varepsilon, r, r_{0}}^{\Delta x} \mathrm{~d} \zeta . \\
\quad+\int_{\mathbb{R}}\left(\operatorname{sign}_{\varepsilon}(\zeta-A(u)) \star J_{r_{0}} \otimes J_{r}\right)\left(\Delta-D_{-} \cdot D_{+}\right) \rho_{\varepsilon, r, r_{0}}^{\Delta x} \mathrm{~d} \zeta \\
\quad-2 \int_{\mathbb{R}}\left(J_{\varepsilon}(\zeta-A(u)) \star J_{r_{0}} \otimes J_{r}\right) n_{G, \varepsilon, r}^{\Delta x} \mathrm{~d} \zeta \\
\quad-2 \int_{\mathbb{R}} E_{\Delta x, \varepsilon, r, r_{0}}(\zeta) \mathrm{d} \zeta,
\end{aligned}
$$

where

$$
\begin{aligned}
E_{\Delta x, \varepsilon, r, r_{0}}(\zeta)= & -\nabla \rho_{\varepsilon, r, r_{0}} \cdot\left(D_{+}+D_{-}\right) \rho_{\varepsilon, r, r_{0}}^{\Delta x} \\
& +\left(J_{\varepsilon}\left(\zeta-A\left(u_{\Delta x}\right)\right) \star J_{r_{0}} \otimes J_{r}\right) n_{A, \varepsilon, r, r_{0}} \\
& +\left(J_{\varepsilon}(\zeta-A(u)) \star J_{r_{0}} \otimes J_{r}\right) n_{A, \varepsilon, r, r_{0}}^{\Delta x} .
\end{aligned}
$$

Proof. By definition,

$$
Q_{\varepsilon, r, r_{0}}(t, x, \zeta)=\operatorname{sign}_{\varepsilon}(\zeta) \rho_{\varepsilon, r, r_{0}}(t, x, \zeta)+\operatorname{sign}_{\varepsilon}(\zeta) \rho_{\varepsilon, r, r_{0}}^{\Delta x}(t, x, \zeta)-2 \rho_{\varepsilon, r, r_{0}}(t, x, \zeta) \rho_{\varepsilon, r, r_{0}}^{\Delta x}(t, x, \zeta)
$$

Hence,

$$
\begin{aligned}
\partial_{t} \int_{\mathbb{R}} Q_{\varepsilon, r, r_{0}} B^{\prime}(\zeta) \mathrm{d} \zeta= & \int_{\mathbb{R}} \operatorname{sign}_{\varepsilon}(\zeta) \partial_{t}\left(\rho_{\varepsilon, r, r_{0}}+\rho_{\varepsilon, r, r_{0}}^{\Delta x}\right) B^{\prime}(\zeta) \mathrm{d} \zeta \\
& +\int_{\mathbb{R}} \partial_{t}\left(\rho_{\varepsilon, r, r_{0}} \rho_{\varepsilon, r, r_{0}}^{\Delta x}\right) B^{\prime}(\zeta) \mathrm{d} \zeta \\
= & : \mathscr{T}_{1}+\mathscr{T}_{2} .
\end{aligned}
$$


By Lemmas 4.7 and 4.8

$$
\begin{aligned}
& \mathscr{T}_{1}=\underbrace{-\int_{\mathbb{R}} \operatorname{sign}_{\varepsilon}(\zeta) g^{\prime}(\zeta) \cdot \nabla\left(\rho_{\varepsilon, r, r_{0}}+\rho_{\varepsilon, r, r_{0}}^{\Delta x}\right) \mathrm{d} \zeta}_{\mathscr{T}_{1}^{1}} \\
& +\underbrace{\int_{\mathbb{R}} \operatorname{sign}_{\varepsilon}(\zeta) \Delta\left(\rho_{\varepsilon, r, r_{0}}+\rho_{\varepsilon, r, r_{0}}^{\Delta x}\right) \mathrm{d} \zeta}_{\mathscr{T}_{1}^{2}} \underbrace{-\int_{\mathbb{R}} \operatorname{sign}_{\varepsilon}(\zeta) \partial_{t}\left(R_{\varepsilon, r, r_{0}}^{B^{\prime}}+R_{\varepsilon, r, r_{0}}^{B^{\prime}, \Delta x}\right) \mathrm{d} \zeta}_{\mathscr{T}_{1}^{3}} \\
& \underbrace{-\int_{\mathbb{R}} \operatorname{sign}_{\varepsilon}(\zeta)\left(\nabla \cdot R_{\varepsilon, r, r_{0}}^{g^{\prime}}+D_{+} \cdot R_{\varepsilon, r, r_{0}}^{G_{1}^{\prime}, \Delta x}+D_{-} \cdot R_{\varepsilon, r, r_{0}}^{G_{2}^{\prime}, \Delta x}\right) \mathrm{d} \zeta}_{\mathscr{T}_{1}^{4}} \\
& +\underbrace{\int_{\mathbb{R}} \operatorname{sign}_{\varepsilon}(\zeta)\left(\partial_{\zeta} n_{A, \varepsilon, r, r_{0}}+\partial_{\zeta} n_{A, \varepsilon, r, r_{0}}^{\Delta x}\right) \mathrm{d} \zeta}_{\mathscr{T}_{1}^{5}} \\
& \underbrace{-\int_{\mathbb{R}} \operatorname{sign}_{\varepsilon}(\zeta)\left(G_{1}^{\prime}(\zeta) \cdot\left(D_{+}-\nabla\right)+G_{2}^{\prime}(\zeta) \cdot\left(D_{-}-\nabla\right)\right) \rho_{\varepsilon, r, r_{0}}^{\Delta x} \mathrm{~d} \zeta}_{\mathscr{T}_{1}^{6}} \\
& +\underbrace{\int_{\mathbb{R}} \operatorname{sign}_{\varepsilon}(\zeta)\left(\Delta-D_{-} \cdot D_{+}\right) \rho_{\varepsilon, r, r_{0}}^{\Delta x} \mathrm{~d} \zeta}_{\mathscr{T}_{1}^{7}}+\underbrace{\int_{\mathbb{R}} \operatorname{sign}_{\varepsilon}(\zeta) \partial_{\zeta} n_{G, \varepsilon, r, r_{0}}^{\Delta x} \mathrm{~d} \zeta}_{\mathscr{T}_{1}^{8}} .
\end{aligned}
$$

Similarly for $\mathscr{T}_{2}$ we obtain

$$
\begin{aligned}
& \mathscr{T}_{2}= \underbrace{2 \int_{\mathbb{R}} g^{\prime}(\zeta) \cdot \nabla\left(\rho_{\varepsilon, r, r_{0}} \rho_{\varepsilon, r, r_{0}}^{\Delta x}\right) \mathrm{d}}_{\mathscr{T}_{2}^{1}} \underbrace{-2 \int_{\mathbb{R}} \rho_{\varepsilon, r, r_{0}} \Delta \rho_{\varepsilon, r, r_{0}}^{\Delta x}+\Delta \rho_{\varepsilon, r, r_{0}} \rho_{\varepsilon, r, r_{0}}^{\Delta x} \mathrm{~d} \zeta}_{\mathscr{T}_{2}^{3}} \\
&+\underbrace{2 \int_{\mathbb{R}} \rho_{\varepsilon, r, r_{0}} \partial_{t} R_{\varepsilon, r, r_{0}}^{B^{\prime}, \Delta x}+\rho_{\varepsilon, r, r_{0}}^{\Delta x} \partial_{t} R_{\varepsilon, r, r_{0}}^{B^{\prime}} \mathrm{d} \zeta}_{\mathscr{T}_{2}^{2}} \\
&+\underbrace{2 \int_{\mathbb{R}} \rho_{\varepsilon, r, r_{0}}^{\Delta x} \nabla \cdot R_{\varepsilon, r, r_{0}}^{g^{\prime}}+\rho_{\varepsilon, r, r_{0}} D_{+} \cdot R_{\varepsilon, r, r_{0}}^{G_{1}^{\prime}, \Delta x}+\rho_{\varepsilon, r, r_{0}} D_{-} \cdot R_{\varepsilon, r, r_{0}}^{G_{2}^{\prime}, \Delta x} \mathrm{~d} \zeta}_{\mathscr{T}_{2}^{4}} \\
& \underbrace{2 \int_{\mathbb{R}} \rho_{\varepsilon, r, r_{0}} \partial_{\zeta} n_{A, \varepsilon, r, r_{0}}^{\Delta x}+\rho_{\varepsilon, r, r_{0}}^{\Delta x} \partial_{\zeta} n_{A, \varepsilon, r, r_{0}} \mathrm{~d} \zeta}_{\mathscr{T}_{2}^{6}} \\
& \underbrace{2 \int_{\mathbb{R}} \rho_{\varepsilon, r, r_{0}}\left(G_{1}^{\prime}(\zeta) \cdot\left(D_{+}-\nabla\right)+G_{2}^{\prime}(\zeta) \cdot\left(D_{-}-\nabla\right)\right) \rho_{\varepsilon, r, r_{0}}^{\Delta x} \mathrm{~d} \zeta}_{\mathscr{T}_{2}^{7}} \\
&-2 \int_{\mathbb{R}} \rho_{\varepsilon, r, r_{0}}\left(\Delta-D_{-} \cdot D_{+}\right) \rho_{\varepsilon, r, r_{0}}^{\Delta x} \mathrm{~d} \zeta \underbrace{-2 \int_{\mathbb{R}} \rho_{\varepsilon, r, r_{0}} \partial_{\zeta} n_{G, \varepsilon, r, r_{0}}^{\Delta x} \mathrm{~d} \zeta}_{\mathscr{T}_{2}^{8}} .
\end{aligned}
$$


We compute $\mathscr{T}_{1}+\mathscr{T}_{2}$ term by term, and thereby explain each of the terms (5.3)-(5.11) in the lemma. We start with

$$
\mathscr{T}_{1}^{1}+\mathscr{T}_{2}^{1}=-\int_{\mathbb{R}} g^{\prime}(\zeta) \cdot \nabla Q_{\varepsilon, r, r_{0}} \mathrm{~d} \zeta,
$$

which gives the last term in (5.2).

To make the second derivative terms a complete derivative we need to add and subtract. Hence we may write

$$
\begin{aligned}
\mathscr{T}_{1}^{2}+\mathscr{T}_{2}^{2}= & \Delta \int_{\mathbb{R}} Q_{\varepsilon, r, r_{0}} \mathrm{~d} \zeta+4 \int_{\mathbb{R}} \nabla \rho_{\varepsilon, r, r_{0}} \cdot \nabla \rho_{\varepsilon, r, r_{0}}^{\Delta x} \mathrm{~d} \zeta \\
= & \Delta \int_{\mathbb{R}} Q_{\varepsilon, r, r_{0}} \mathrm{~d} \zeta+2 \int_{\mathbb{R}} \nabla \rho_{\varepsilon, r, r_{0}} \cdot\left(D_{+}+D_{-}\right) \rho_{\varepsilon, r, r_{0}}^{\Delta x} \mathrm{~d} \zeta \\
& +2 \int_{\mathbb{R}} \nabla \rho_{\varepsilon, r, r_{0}}\left(2 \nabla-\left(D_{+}+D_{-}\right)\right) \rho_{\varepsilon, r, r_{0}}^{\Delta x} \mathrm{~d} \zeta,
\end{aligned}
$$

which explains (5.3) and the first term in $E_{\Delta x, \varepsilon, r, r_{0}}$.

By Lemma 4.6 it follows that

$$
\begin{aligned}
& \operatorname{sign}_{\varepsilon}(\zeta)-2 \rho_{\varepsilon, r, r_{0}}=\operatorname{sign}_{\varepsilon}(\zeta-A(u)) \star J_{r_{0}} \otimes J_{r}, \\
& \operatorname{sign}_{\varepsilon}(\zeta)-2 \rho_{\varepsilon, r, r_{0}}^{\Delta x}=\operatorname{sign}_{\varepsilon}\left(\zeta-A\left(u_{\Delta x}\right)\right) \star J_{r_{0}} \otimes J_{r} .
\end{aligned}
$$

Hence,

$$
\begin{aligned}
\mathscr{T}_{1}^{3}+\mathscr{T}_{2}^{3}= & -\int_{\mathbb{R}}\left(\operatorname{sign}_{\varepsilon}\left(\zeta-A\left(u_{\Delta x}\right)\right) \star J_{r_{0}} \otimes J_{r}\right) \partial_{t} R_{\varepsilon, r, r_{0}}^{B^{\prime}} \mathrm{d} \zeta \\
& -\int_{\mathbb{R}}\left(\operatorname{sign}_{\varepsilon}(\zeta-A(u)) \star J_{r_{0}} \otimes J_{r}\right) \partial_{t} R_{\varepsilon, r, r_{0}}^{B^{\prime}, \Delta x} \mathrm{~d} \zeta,
\end{aligned}
$$

which explains (5.4) and (5.5) Similarly,

$$
\begin{aligned}
\mathscr{T}_{1}^{4}+\mathscr{T}_{2}^{4}= & -\int_{\mathbb{R}}\left(\operatorname{sign}_{\varepsilon}\left(\zeta-A\left(u_{\Delta x}\right)\right) \star J_{r_{0}} \otimes J_{r}\right) \nabla \cdot R_{\varepsilon, r, r_{0}}^{g^{\prime}} \mathrm{d} \zeta \\
& -\int_{\mathbb{R}}\left(\operatorname{sign}_{\varepsilon}(\zeta-A(u)) \star J_{r_{0}} \otimes J_{r}\right)\left(D_{+} \cdot R_{\varepsilon, r, r_{0}}^{G_{1}^{\prime}, \Delta x}+D_{-} \cdot R_{\varepsilon, r, r_{0}}^{G_{2}^{\prime}, \Delta x}\right) \mathrm{d} \zeta,
\end{aligned}
$$

which explains the presence of (5.6) and (5.7).

Performing integration by parts we obtain, using Lemma 4.6,

$$
\begin{aligned}
\mathscr{T}_{1}^{5}+\mathscr{T}_{2}^{5}= & \int_{\mathbb{R}}\left(\operatorname{sign}_{\varepsilon}\left(\zeta-A\left(u_{\Delta x}\right)\right) \star J_{r_{0}} \otimes J_{r}\right) \partial_{\zeta} n_{A, \varepsilon, r, r_{0}} \\
& +\left(\operatorname{sign}_{\varepsilon}(\zeta-A(u)) \star J_{r_{0}} \otimes J_{r}\right) \partial_{\zeta} n_{A, \varepsilon, r, r_{0}}^{\Delta x} \mathrm{~d} \zeta \\
= & -2 \int_{\mathbb{R}}\left(J_{\varepsilon}\left(\zeta-A\left(u_{\Delta x}\right)\right) \star J_{r_{0}} \otimes J_{r}\right) n_{A, \varepsilon, r, r_{0}} \\
& +\left(J_{\varepsilon}(\zeta-A(u)) \star J_{r_{0}} \otimes J_{r}\right) n_{A, \varepsilon, r, r_{0}}^{\Delta x} \mathrm{~d} \zeta,
\end{aligned}
$$

which explains the two last terms in $E_{\Delta x, \varepsilon, r, r_{0}}$.

Similarly,

$$
\begin{aligned}
\mathscr{T}_{1}^{6}+\mathscr{T}_{2}^{6}= & -\int_{\mathbb{R}}\left(\operatorname{sign}_{\varepsilon}(\zeta-A(u)) \star J_{r_{0}} \otimes J_{r}\right)\left(G_{1}^{\prime}(\zeta) \cdot\left(D_{+}-\nabla\right)\right. \\
& \left.+G_{2}^{\prime}(\zeta) \cdot\left(D_{-}-\nabla\right)\right) \rho_{\varepsilon, r, r_{0}}^{\Delta x} \mathrm{~d} \zeta
\end{aligned}
$$


and

$$
\mathscr{T}_{1}^{7}+\mathscr{T}_{2}^{7}=\int_{\mathbb{R}}\left(\operatorname{sign}_{\varepsilon}(\zeta-A(u)) \star J_{r_{0}} \otimes J_{r}\right)\left(\Delta-D_{-} \cdot D_{+}\right) \rho_{\varepsilon, r, r_{0}}^{\Delta x} \mathrm{~d} \zeta,
$$

explaining the terms (5.8) and (5.9).

Finally, integration by parts yields

$$
\mathscr{T}_{1}^{8}+\mathscr{T}_{2}^{8}=-2 \int_{\mathbb{R}}\left(J_{\varepsilon}(\zeta-A(u)) \star J_{r_{0}} \otimes J_{r}\right) n_{G, \varepsilon, r, r_{0}}^{\Delta x} \mathrm{~d} \zeta,
$$

which is the term (5.10).

\subsection{Dissipative term}

In this subsection we are concerned with finding an upper bound on (5.12). In the continuous setting, this "dissipative" term is negative, $c f$. (1.13), which comes as a consequence of the chain rule of calculus. The following elementary lemma will help us contend with the lack of a discrete chain rule.

Lemma 5.2. Let $a$ and $b$ be two real numbers. Then there exist real numbers $\tau=\tau_{\varepsilon}(a, b, \zeta)$ and $\theta=\theta_{\varepsilon}(a, b, \zeta)$ such that $\tau$ and $\theta$ are between $a$ and $b$, and

$$
\begin{gathered}
\int_{\mathbb{R}} J_{\varepsilon}(\zeta-\xi)(\chi(b ; \xi)-\chi(a ; \xi)) \mathrm{d} \xi=J_{\varepsilon}(\zeta-\theta)(b-a), \\
\int_{\mathbb{R}} J_{\varepsilon}(\zeta-\xi)(b-\xi)(\chi(b ; \xi)-\chi(a ; \xi)) \mathrm{d} \xi=\frac{1}{2} J_{\varepsilon}(\zeta-\tau)(b-a)^{2} .
\end{gathered}
$$

Furthermore, whenever $a \neq b$ :

(i)

$$
\begin{aligned}
& J_{\varepsilon}(\zeta-\theta)=\frac{1}{b-a} \int_{a}^{b} J_{\varepsilon}(\zeta-\xi) \mathrm{d} \xi \\
& J_{\varepsilon}(\zeta-\tau)=\frac{2}{(b-a)^{2}} \int_{a}^{b} J_{\varepsilon}(\zeta-\xi)(b-\xi) \mathrm{d} \xi
\end{aligned}
$$

(ii)

$$
\left(J_{\varepsilon}(\zeta-\theta)-J_{\varepsilon}(\zeta-\tau)\right)(b-a)=\frac{1}{b-a} \int_{a}^{b} J_{\varepsilon}(\zeta-\xi)(2 \xi-(b+a)) \mathrm{d} \xi
$$

$$
J_{\varepsilon}(\zeta-\tau)-J_{\varepsilon}(\zeta-a)=\frac{2}{(b-a)^{2}}\left(\int_{a}^{b}\left(J_{\varepsilon}(\zeta-\xi)-J_{\varepsilon}(\zeta-a)\right)(b-\xi) \mathrm{d} \xi\right)
$$

$$
J_{\varepsilon}\left(\zeta-\theta_{\varepsilon}(a, b, \zeta)\right)-J_{\varepsilon}\left(\zeta-\tau_{\varepsilon}(a, b, \zeta)\right)=-\left(J_{\varepsilon}\left(\zeta-\theta_{\varepsilon}(b, a, \zeta)\right)-J_{\varepsilon}\left(\zeta-\tau_{\varepsilon}(b, a, \zeta)\right)\right) .
$$

Proof. To prove (5.14), note that

$$
\int_{\mathbb{R}} J_{\varepsilon}(\zeta-\xi)(b-\xi)(\chi(b ; \xi)-\chi(a ; \xi)) \mathrm{d} \xi=\int_{a}^{b} J_{\varepsilon}(\zeta-\xi)(b-\xi) \mathrm{d} \xi .
$$

By the mean value theorem there exists a $\tau$ between $a$ and $b$ such that

$$
\int_{a}^{b} J_{\varepsilon}(\zeta-\xi)(b-\xi) \mathrm{d} \xi=J_{\varepsilon}(\zeta-\tau) \int_{a}^{b}(b-\xi) \mathrm{d} \xi
$$


Equation (5.13) follows in a similar way. The proof of (i) is immediate. Let us prove (ii). By (i)

$$
\left(J_{\varepsilon}(\zeta-\theta)-J_{\varepsilon}(\zeta-\tau)\right)(b-a)=\int_{a}^{b} J_{\varepsilon}(\zeta-\xi)\left(1-2 \frac{b-\xi}{b-a}\right) \mathrm{d} \xi
$$

It remains to observe that

$$
1-2 \frac{b-\xi}{b-a}=\frac{2 \xi-(b+a)}{b-a}
$$

To prove (iii), note that

$$
J_{\varepsilon}(\zeta-a)(b-a)^{2}=2 \int_{a}^{b} J_{\varepsilon}(\zeta-a)(b-\xi) \mathrm{d} \xi .
$$

Hence (iii) follows by (i). To prove (iv), observe that the expression on the right-hand side of (ii) is symmetric in $a$ and $b$.

The next result can be viewed as a discrete counterpart of the the chain rule, enabling us to write the nonlinear term $n_{A}^{\Delta x}$, properly regularized, on a form that resembles a parabolic dissipation term like (1.14).

Lemma 5.3. With the notation of Lemma 4.5, for each $1 \leq i \leq d$, let

$$
\tau_{\Delta x, i}^{+}=\tau_{\varepsilon}\left(A\left(u_{\Delta x}\right), S_{\Delta x_{i}} A\left(u_{\Delta x}\right), \zeta\right), \quad \tau_{\Delta x, i}^{-}=\tau_{\varepsilon}\left(A\left(u_{\Delta x}\right), S_{-\Delta x_{i}} A\left(u_{\Delta x}\right), \zeta\right)
$$

and

$$
\theta_{\Delta x, i}^{+}=\theta_{\varepsilon}\left(A\left(u_{\Delta x}\right), S_{\Delta x_{i}} A\left(u_{\Delta x}\right), \zeta\right), \quad \theta_{\Delta x, i}^{-}=\theta_{\varepsilon}\left(A\left(u_{\Delta x}\right), S_{-\Delta x_{i}} A\left(u_{\Delta x}\right), \zeta\right),
$$

where $\tau_{\varepsilon}, \theta_{\varepsilon}$ is defined in Lemma 5.2. Then

(i)

$$
n_{A}^{\Delta x} \star J_{\varepsilon}(t, x, \zeta)=\frac{1}{2} \sum_{i=1}^{d} J_{\varepsilon}\left(\zeta-\tau_{\Delta x, i}^{+}\right)\left(D_{+}^{i} A\left(u_{\Delta x}\right)\right)^{2}+\frac{1}{2} \sum_{i=1}^{d} J_{\varepsilon}\left(\zeta-\tau_{\Delta x, i}^{-}\right)\left(D_{-}^{i} A\left(u_{\Delta x}\right)\right)^{2} ;
$$

(ii) for $1 \leq i \leq d$,

$$
\begin{aligned}
& D_{+}^{i} \chi_{\varepsilon}\left(A\left(u_{\Delta x}\right) ; \zeta\right)=J_{\varepsilon}\left(\zeta-\theta_{\Delta x, i}^{+}\right) D_{+}^{i}\left(A\left(u_{\Delta x}\right)\right), \\
& D_{-}^{i} \chi_{\varepsilon}\left(A\left(u_{\Delta x}\right) ; \zeta\right)=J_{\varepsilon}\left(\zeta-\theta_{\Delta x, i}^{-}\right) D_{-}^{i}\left(A\left(u_{\Delta x}\right)\right) .
\end{aligned}
$$

Proof. By the definition (4.4) of $n_{A}^{\Delta x}$, recalling that $S_{y}$ commutes with function evaluation,

$$
\begin{aligned}
n_{A}^{\Delta x} \star J_{\varepsilon}(t, x, \zeta)= & \sum_{i=1}^{d} \frac{1}{\Delta x^{2}} \int_{\mathbb{R}} J_{\varepsilon}(\zeta-\xi)\left(S_{\Delta x_{i}} A\left(u_{\Delta x}\right)-\xi\right)\left(\chi\left(S_{\Delta x_{i}} A\left(u_{\Delta x}\right) ; \xi\right)-\chi\left(A\left(u_{\Delta x}\right) ; \xi\right)\right) \mathrm{d} \xi \\
& +\sum_{i=1}^{d} \frac{1}{\Delta x^{2}} \int_{\mathbb{R}} J_{\varepsilon}(\zeta-\xi)\left(S_{-\Delta x_{i}} A\left(u_{\Delta x}\right)-\xi\right)\left(\chi\left(S_{-\Delta x_{i}} A\left(u_{\Delta x}\right) ; \xi\right)-\chi\left(A\left(u_{\Delta x}\right) ; \xi\right)\right) \mathrm{d} \xi .
\end{aligned}
$$

Hence (i) follows by Lemma 5.2. To prove (ii) note that by Lemma 5.2 ,

$$
\begin{aligned}
D_{+}^{i} \int_{\mathbb{R}} \chi\left(A\left(u_{\Delta x}\right) ; \xi\right) J_{\varepsilon}(\zeta-\xi) \mathrm{d} \xi & =\frac{1}{\Delta x} \int_{\mathbb{R}} J_{\varepsilon}(\zeta-\xi)\left(\chi\left(S_{\Delta x_{i}} A\left(u_{\Delta x}\right) ; \xi\right)-\chi\left(A\left(u_{\Delta x}\right) ; \xi\right)\right) \mathrm{d} \xi \\
& =J_{\varepsilon}\left(\zeta-\theta_{\Delta x, i}^{+}\right) D_{+}^{i}\left(A\left(u_{\Delta x}\right)\right) .
\end{aligned}
$$

The same argument applies to $\theta_{\Delta x, i}^{-}$. 
We have now come to the key result of this subsection, namely a lower bound on the discrete dissipation term (5.12).

Lemma 5.4. Let $E_{\Delta x, \varepsilon, r, r_{0}}$ be defined in Lemma 5.1. Then

$$
E_{\Delta x, \varepsilon, r, r_{0}} \geq \sum_{k=1}^{2}\left(R_{k}^{+}+R_{k}^{-}\right) \text {everywhere in }\left(r_{0}, T-r_{0}\right) \times \mathbb{R}^{d} \times \mathbb{R},
$$

for all positive numbers $\Delta x, \varepsilon, r$, and $r_{0}$, where

$$
\begin{aligned}
R_{1}^{+}(\zeta)= & \sum_{i=1}^{d}\left(\left(J_{\varepsilon}\left(\zeta-\tau_{\Delta x, i}^{+}\right)-J_{\varepsilon}\left(\zeta-\theta_{\Delta x, i}^{+}\right)\right) D_{+}^{i} A\left(u_{\Delta x}\right) \star J_{r_{0}} \otimes J_{r}\right) \partial_{x_{i}} \rho_{\varepsilon, r, r_{0}} \\
R_{1}^{-}(\zeta)= & \left.\sum_{i=1}^{d}\left(\left(J_{\varepsilon}\left(\zeta-\tau_{\Delta x, i}^{-}\right)-J_{\varepsilon}\left(\zeta-\theta_{\Delta x, i}^{-}\right)\right) D_{-}^{i} A\left(u_{\Delta x}\right)\right) \star J_{r_{0}} \otimes J_{r}\right) \partial_{x_{i}} \rho_{\varepsilon, r, r_{0}} \\
R_{2}^{+}(\zeta)= & \frac{1}{2} \sum_{i=1}^{d}\left[\left(\left(J_{\varepsilon}\left(\zeta-A\left(u_{\Delta x}\right)\right)-J_{\varepsilon}\left(\zeta-A\left(\tau_{\Delta x, i}^{+}\right)\right) \star J_{r_{0}} \otimes J_{r}\right)\right.\right. \\
& \left.\times\left(J_{\varepsilon}(\zeta-A(u))\left(\partial_{x_{i}} A(u)\right)^{2} \star J_{r_{0}} \otimes J_{r}\right)\right] \\
R_{2}^{-}(\zeta)=\frac{1}{2} \sum_{i=1}^{d}\left[\left(\left(J_{\varepsilon}\left(\zeta-A\left(u_{\Delta x}\right)\right)-\right.\right.\right. & \left.J_{\varepsilon}\left(\zeta-A\left(\tau_{\Delta x, i}^{-}\right)\right) \star J_{r_{0}} \otimes J_{r}\right) \\
\times & \left.\times\left(J_{\varepsilon}(\zeta-A(u))\left(\partial_{x_{i}} A(u)\right)^{2} \star J_{r_{0}} \otimes J_{r}\right)\right]
\end{aligned}
$$

Proof. By Lemma 5.3,

$$
\begin{aligned}
\left(J_{\varepsilon}(\zeta-A(u)) \star J_{r_{0}} \otimes J_{r}\right) n_{A, \varepsilon, r, r_{0}}^{\Delta x}= & \frac{1}{2} \sum_{i=1}^{d}\left(J_{\varepsilon}(\zeta-A(u)) \star J_{r_{0}} \otimes J_{r}\right)\left(J_{\varepsilon}\left(\zeta-\tau_{\Delta x, i}^{+}\right)\left(D_{+}^{i} A\left(u_{\Delta x}\right)\right)^{2} \star J_{r_{0}} \otimes J_{r}\right) \\
& +\frac{1}{2} \sum_{i=1}^{d}\left(J_{\varepsilon}(\zeta-A(u)) \star J_{r_{0}} \otimes J_{r}\right)\left(J_{\varepsilon}\left(\zeta-\tau_{\Delta x, i}^{-}\right)\left(D_{-}^{i} A\left(u_{\Delta x}\right)\right)^{2} \star J_{r_{0}} \otimes J_{r}\right) \\
= & : \mathscr{T}_{1}^{+}+\mathscr{T}_{1}^{-} .
\end{aligned}
$$

Observe that

$$
\partial_{x_{i}} \rho_{\varepsilon, r, r_{0}}=\partial_{x_{i}}\left(\chi_{\varepsilon}(A(u) ; \zeta) \star J_{r_{0}} \otimes J_{r}\right)=J_{\varepsilon}(\zeta-A(u)) \partial_{x_{i}} A(u) \star J_{r_{0}} \otimes J_{r} .
$$

Using Lemma 5.3 once more gives

$$
\begin{aligned}
\left(D_{+}^{i}+D_{-}^{i}\right) \rho_{\varepsilon, r, r_{0}}^{\Delta x}= & \left(J_{\varepsilon}\left(\zeta-\theta_{\Delta x, i}^{+}\right) D_{+}^{i} A\left(u_{\Delta x}\right) \star J_{r_{0}} \otimes J_{r}\right. \\
& \left.+J_{\varepsilon}\left(\zeta-\theta_{\Delta x, i}^{-}\right) D_{-}^{i} A\left(u_{\Delta x}\right)\right) \star J_{r_{0}} \otimes J_{r} .
\end{aligned}
$$

Hence,

$$
\begin{aligned}
\nabla \rho_{\varepsilon, r, r_{0}} \cdot\left(D_{+}+D_{-}\right) \rho_{\varepsilon, r, r_{0}}^{\Delta x}= & \sum_{i=1}^{d}\left(J_{\varepsilon}(\zeta-A(u)) \partial_{x_{i}} A(u) \star J_{r_{0}} \otimes J_{r}\right)\left(J_{\varepsilon}\left(\zeta-\theta_{\Delta x, i}^{+}\right) D_{+}^{i} A\left(u_{\Delta x}\right) \star J_{r_{0}} \otimes J_{r}\right) \\
& \left.+\sum_{i=1}^{d}\left(J_{\varepsilon}(\zeta-A(u)) \partial_{x_{i}} A(u) \star J_{r_{0}} \otimes J_{r}\right)\left(J_{\varepsilon}\left(\zeta-\theta_{\Delta x, i}^{-}\right) D_{-}^{i} A\left(u_{\Delta x}\right)\right) \star J_{r_{0}} \otimes J_{r}\right) .
\end{aligned}
$$


Adding and subtracting we obtain

$$
-\nabla \rho_{\varepsilon, r, r_{0}} \cdot\left(D_{+}+D_{-}\right) \rho_{\varepsilon, r, r_{0}}^{\Delta x}=\mathscr{T}_{2}^{+}+\mathscr{T}_{2}^{-}+R_{1}^{+}+R_{1}^{-},
$$

where

$$
\begin{aligned}
& \mathscr{T}_{2}^{+}=-\sum_{i=1}^{d}\left(J_{\varepsilon}(\zeta-A(u)) \partial_{x_{i}} A(u) \star J_{r_{0}} \otimes J_{r}\right)\left(J_{\varepsilon}\left(\zeta-\tau_{\Delta x, i}^{+}\right) D_{+}^{i} A\left(u_{\Delta x}\right) \star J_{r_{0}} \otimes J_{r}\right), \\
& \left.\mathscr{T}_{2}^{-}=-\sum_{i=1}^{d}\left(J_{\varepsilon}(\zeta-A(u)) \partial_{x_{i}} A(u) \star J_{r_{0}} \otimes J_{r}\right)\left(J_{\varepsilon}\left(\zeta-\tau_{\Delta x, i}^{-}\right) D_{-}^{i} A\left(u_{\Delta x}\right)\right) \star J_{r_{0}} \otimes J_{r}\right) .
\end{aligned}
$$

For each $1 \leq i \leq d$,

$$
\begin{aligned}
J_{\varepsilon}\left(\zeta-A\left(u_{\Delta x}\right)\right)= & \frac{1}{2}\left(J_{\varepsilon}\left(\zeta-A\left(u_{\Delta x}\right)\right)-J_{\varepsilon}\left(\zeta-A\left(\tau_{\Delta x, i}^{+}\right)\right)\right) \\
& +\frac{1}{2}\left(J_{\varepsilon}\left(\zeta-A\left(u_{\Delta x}\right)\right)-J_{\varepsilon}\left(\zeta-A\left(\tau_{\Delta x, i}^{-}\right)\right)\right) \\
& +\frac{1}{2}\left(J_{\varepsilon}\left(\zeta-A\left(\tau_{\Delta x, i}^{+}\right)\right)+J_{\varepsilon}\left(\zeta-A\left(\tau_{\Delta x, i}^{-}\right)\right)\right) .
\end{aligned}
$$

It follows that

$$
\left(J_{\varepsilon}\left(\zeta-A\left(u_{\Delta x}\right)\right) \star J_{r_{0}} \otimes J_{r}\right) n_{A, \varepsilon, r, r_{0}}=\mathscr{T}_{3}^{+}+\mathscr{T}_{3}^{-}+R_{2}^{+}+R_{2}^{-},
$$

where

$$
\begin{aligned}
& \mathscr{T}_{3}^{+}=\frac{1}{2} \sum_{i=1}^{d}\left(J_{\varepsilon}\left(\zeta-A\left(\tau_{\Delta x, i}^{+}\right)\right) \star J_{r_{0}} \otimes J_{r}\right)\left(J_{\varepsilon}(\zeta-A(u))\left(\partial_{x_{i}} A(u)\right)^{2} \star J_{r_{0}} \otimes J_{r}\right), \\
& \mathscr{T}_{3}^{-}=\frac{1}{2} \sum_{i=1}^{d}\left(J_{\varepsilon}\left(\zeta-A\left(\tau_{\Delta x, i}^{-}\right)\right) \star J_{r_{0}} \otimes J_{r}\right)\left(J_{\varepsilon}(\zeta-A(u))\left(\partial_{x_{i}} A(u)\right)^{2} \star J_{r_{0}} \otimes J_{r}\right) .
\end{aligned}
$$

Note that

$$
E_{\Delta x, \varepsilon, r, r_{0}}=\sum_{k=1}^{3}\left(\mathscr{T}_{k}^{+}+\mathscr{T}_{k}^{-}\right)+\sum_{k=1}^{2}\left(R_{k}^{+}+R_{k}^{-}\right)
$$

Now,

$$
\begin{aligned}
\sum_{k=1}^{3} \mathscr{T}_{k}^{+}= & \frac{1}{2} \sum_{i=1}^{d}\left(J_{\varepsilon}(\zeta-A(u)) \star J_{r_{0}} \otimes J_{r}\right)\left(\left(J_{\varepsilon}\left(\zeta-\tau_{\Delta x, i}^{+}\right)\left(D_{+}^{i} A\left(u_{\Delta x}\right)\right)^{2} \star J_{r_{0}} \otimes J_{r}\right)\right. \\
& -\sum_{i=1}^{d}\left(J_{\varepsilon}(\zeta-A(u)) \partial_{x_{i}} A(u) \star J_{r_{0}} \otimes J_{r}\right)\left(J_{\varepsilon}\left(\zeta-\tau_{\Delta x, i}^{+}\right) D_{+}^{i} A\left(u_{\Delta x}\right) \star J_{r_{0}} \otimes J_{r}\right) \\
& +\frac{1}{2} \sum_{i=1}^{d}\left(J_{\varepsilon}\left(\zeta-A\left(\tau_{\Delta x, i}^{+}\right)\right) \star J_{r_{0}} \otimes J_{r}\right)\left(J_{\varepsilon}(\zeta-A(u))\left(\partial_{x_{i}} A(u)\right)^{2} \star J_{r_{0}} \otimes J_{r}\right) \\
= & \frac{1}{2} \sum_{i=1}^{d} J_{\varepsilon}(\zeta-A(u)) J_{\varepsilon}\left(\zeta-A\left(\tau_{\Delta x, i}^{+}\right)\right)\left(\partial_{x_{i}} A(u)-D_{+}^{i} A\left(u_{\Delta x}\right)\right)^{2} \\
& \stackrel{\left(u, u_{\Delta x}\right)}{\star} J_{r_{0}} \otimes J_{r} \otimes J_{r_{0}} \otimes J_{r} \\
\geq & 0 .
\end{aligned}
$$


The obtained inequality holds for all $(t, x, \zeta) \in\left(r_{0}, T-r_{0}\right) \times \mathbb{R}^{d} \times \mathbb{R}$. Similarly,

$$
\sum_{i=1}^{3} \mathscr{T}_{i}^{-}(t, x, \zeta) \geq 0 \text { for all }(t, x, \zeta) \in\left(r_{0}, T-r_{0}\right) \times \mathbb{R}^{d} \times \mathbb{R} .
$$

This concludes the proof of the lemma.

\subsection{Bounding error terms}

We are going to estimate a series of "unwanted" terms coming from Lemmas 5.1 and 5.4. To this end, we will need to gather three technical lemmas, the first one being a simple application of Young's inequality for convolutions.

Lemma 5.5. Let $\psi: \mathbb{R}^{2} \rightarrow \mathbb{R}$ be a measurable function, and $u, v: \mathbb{R}^{d} \rightarrow \mathbb{R}$ be measurable functions satisfying

$$
\left|\psi\left(u\left(x_{1}\right), v\left(x_{2}\right)\right)\right| \leq K_{1}\left(x_{1}\right) K_{2}\left(x_{2}\right) \quad\left(x_{1}, x_{2} \in \mathbb{R}^{d}\right),
$$

for some $K_{1} \in L^{p}\left(\mathbb{R}^{d}\right), 1 \leq p \leq \infty$, and $K_{2} \in L^{1}\left(\mathbb{R}^{d}\right)$. Then

$$
\|\psi(u, v) \stackrel{(u, v)}{\star} f \otimes g\|_{L^{1}\left(\mathbb{R}^{d}\right)} \leq\left\|K_{1}\right\|_{L^{p}\left(\mathbb{R}^{d}\right)}\left\|K_{2}\right\|_{L^{1}\left(\mathbb{R}^{d}\right)}\|f\|_{L^{q}\left(\mathbb{R}^{d}\right)}\|g\|_{L^{1}\left(\mathbb{R}^{d}\right)},
$$

for any $g \in L^{1}(\mathbb{R})$ and $f \in L^{q}(\mathbb{R})$ where $p^{-1}+q^{-1}=1$.

If $\psi \in L^{\infty}\left(\mathbb{R}^{2}\right)$, then

$$
\|\psi(u, v) \stackrel{(u, v)}{\star} f \otimes g\|_{L^{\infty}\left(\mathbb{R}^{d}\right)} \leq\|\psi\|_{L^{\infty}\left(\mathbb{R}^{2}\right)}\|f\|_{L^{1}\left(\mathbb{R}^{d}\right)}\|g\|_{L^{1}\left(\mathbb{R}^{d}\right)} .
$$

Proof. Observe that

$$
\begin{aligned}
\|\psi(u, v) \stackrel{(u, v)}{\star} f \otimes g\|_{L^{1}\left(\mathbb{R}^{d}\right)} \leq & \iiint_{\mathbb{R}^{d} \times \mathbb{R}^{d} \times \mathbb{R}^{d}} K_{1}\left(y_{1}\right) K_{2}\left(y_{2}\right)\left|f\left(x-y_{1}\right)\right|\left|g\left(x-y_{2}\right)\right| \mathrm{d} y_{1} \mathrm{~d} y_{2} \mathrm{~d} x \\
& =\left\|\left(K_{1} \star|f|\right)\left(K_{2} \star|g|\right)\right\|_{L^{1}\left(\mathbb{R}^{d}\right)} .
\end{aligned}
$$

By Hölder's inequality,

$$
\left\|\left(K_{1} \star|f|\right)\left(K_{2} \star|g|\right)\right\|_{L^{1}\left(\mathbb{R}^{d}\right)} \leq\left\|K_{1} \star|f|\right\|_{L^{\infty}\left(\mathbb{R}^{d}\right)}\left\|K_{2} \star|g|\right\|_{L^{1}\left(\mathbb{R}^{d}\right)} .
$$

By Young's inequality for convolutions, $\left\|K_{1} \star|f|\right\|_{L^{\infty}\left(\mathbb{R}^{d}\right)} \leq\left\|K_{1}\right\|_{L^{p}\left(\mathbb{R}^{d}\right)}\|f\|_{L^{q}\left(\mathbb{R}^{d}\right)}$ and $\left\|K_{2} \star|g|\right\|_{L^{1}\left(\mathbb{R}^{d}\right)} \leq$ $\left\|K_{2}\right\|_{L^{1}\left(\mathbb{R}^{d}\right)}\|g\|_{L^{1}\left(\mathbb{R}^{d}\right)}$. Equation (5.15) follows, since

$$
\left|\int_{\mathbb{R}^{d}} \int_{\mathbb{R}^{d}} \psi\left(u\left(y_{1}\right), v\left(y_{2}\right)\right) f\left(x-y_{1}\right) g\left(x-y_{2}\right) \mathrm{d} y_{1} \mathrm{~d} y_{2}\right| \leq\|\psi\|_{L^{\infty}\left(\mathbb{R}^{2}\right)}\|f\|_{L^{1}\left(\mathbb{R}^{d}\right)}\|g\|_{L^{1}\left(\mathbb{R}^{d}\right)} .
$$

The next lemma is at the heart of the matter, permitting us to estimate some terms involving convolutions against approximate delta functions.

Lemma 5.6. For real numbers $a$ and $b$, let $\tau=\tau_{\varepsilon}(a, b, \zeta)$ and $\theta=\theta_{\varepsilon}(a, b, \zeta)$ be as in Lemma 5.2.

(i) For $f \in L_{\text {loc }}^{1}(\mathbb{R})$, define

$$
\mathscr{T}_{\varepsilon}^{1}(f)=\int_{\mathbb{R}}\left(J_{\varepsilon}(\zeta-\tau)-J_{\varepsilon}(\zeta-a)\right) f(\zeta) \mathrm{d} \zeta .
$$

Then

$$
\left|\mathscr{T}_{\varepsilon}^{1}(f)\right| \leq\left|\int_{a}^{b}\right| \partial_{\xi}\left(f \star J_{\varepsilon}\right)(\xi)|\mathrm{d} \xi| .
$$


(ii) For $f \in L^{\infty}(\mathbb{R})$, define

$$
\mathscr{T}_{\varepsilon}^{2}(f)=\int_{\mathbb{R}}\left(J_{\varepsilon}(\zeta-\theta)-J_{\varepsilon}(\zeta-\tau)\right)(b-a) f(\zeta) \mathrm{d} \zeta
$$

Then

$$
\left|\mathscr{T}_{\varepsilon}^{2}(f)\right| \leq \frac{1}{2}\|f\|_{L^{\infty}(\mathbb{R})}|b-a| .
$$

(iii) Suppose $\left\{f_{\varepsilon}\right\}_{\varepsilon>0} \subset W_{\mathrm{loc}}^{1,1}(\mathbb{R})$ and assume that there exists $f \in W_{\mathrm{loc}}^{1,1}(\mathbb{R})$ such that $f_{\varepsilon} \rightarrow f$ in $W_{\mathrm{loc}}^{1,1}(\mathbb{R})$. Then

$$
\left|\lim _{\varepsilon \downarrow 0} \mathscr{T}_{\varepsilon}^{1}\left(f_{\varepsilon}\right)\right| \leq\left|\int_{a}^{b}\right| f^{\prime}(\xi)|\mathrm{d} \xi| .
$$

Proof. Assume that $a<b$. By Lemma 5.2 and Fubini's theorem,

$$
\mathscr{T}_{\varepsilon}^{1}(f)=\frac{2}{(b-a)^{2}} \int_{a}^{b}\left(\left(f \star J_{\varepsilon}\right)(\xi)-\left(f \star J_{\varepsilon}\right)(a)\right)(b-\xi) \mathrm{d} \xi .
$$

Since $\partial_{\xi}(b-\xi)^{2}=-2(b-\xi)$, integration by parts yields

$$
\mathscr{T}_{\varepsilon}^{1}(f)=\int_{a}^{b} \partial_{\xi}\left(f \star J_{\varepsilon}\right)(\xi) \frac{(b-\xi)^{2}}{(b-a)^{2}} \mathrm{~d} \xi .
$$

Then statement (i) follows, since

$$
\frac{(b-\xi)^{2}}{(b-a)^{2}} \leq 1, \text { whenever } a \leq \xi \leq b .
$$

By Lemma 5.2,

$$
\mathscr{T}_{\varepsilon}^{2}(f)=\frac{1}{b-a} \int_{a}^{b}\left(f \star J_{\varepsilon}\right)(\xi)(2 \xi-(a+b)) \mathrm{d} \xi .
$$

As $\left\|f \star J_{\varepsilon}\right\|_{L^{\infty}(\mathbb{R})} \leq\|f\|_{L^{\infty}(\mathbb{R})}$, we may conclude that

$$
\mathscr{T}_{\varepsilon}^{2}(f) \leq \frac{1}{b-a} \int_{a}^{b}\|f\|_{L^{\infty}(\mathbb{R})}|(2 \xi-(a+b))| \mathrm{d} \xi .
$$

This implies statement (ii), because

$$
\int_{a}^{b}|2 \xi-(a+b)| \mathrm{d} \xi=\frac{1}{2}(b-a)^{2} .
$$

Finally, we establish statement (iii). By the triangle inequality and Young's inequality for convolutions,

$$
\left\|f_{\varepsilon}^{\prime} \star J_{\varepsilon}-f^{\prime}\right\|_{L^{1}(V)} \leq\left\|f_{\varepsilon}^{\prime}-f^{\prime}\right\|_{L^{1}(V)}+\left\|f^{\prime} \star J_{\varepsilon}-f^{\prime}\right\|_{L^{1}(V)}
$$

for any compact $V \subset \mathbb{R}$. Hence $\left(f_{\varepsilon}^{\prime} \star J_{\varepsilon}\right) \rightarrow f^{\prime}$ in $L_{\text {loc }}^{1}(\mathbb{R})$ as $\varepsilon \downarrow 0$. By (5.16) and (5.17) it follows that

$$
\lim _{\varepsilon \downarrow 0} \mathscr{T}_{\varepsilon}^{1}\left(f_{\varepsilon}\right)=\lim _{\varepsilon \downarrow 0} \int_{a}^{b}\left(f_{\varepsilon}^{\prime} \star J_{\varepsilon}\right)(\xi) \frac{(b-\xi)^{2}}{(b-a)^{2}} \mathrm{~d} \xi=\int_{a}^{b} f^{\prime}(\xi) \frac{(b-\xi)^{2}}{(b-a)^{2}} \mathrm{~d} \xi .
$$

The estimate follows thanks to (5.17).

We need one more lemma bounding some specific convolution integrals. 
Lemma 5.7. Suppose $f \in C(\mathbb{R})$ and let $R_{\varepsilon}^{f}: \mathbb{R}^{2} \rightarrow \mathbb{R}$ be defined by (4.5). Then

$$
R_{\varepsilon}^{f}(u, \zeta)=\int_{0}^{u}(f(\sigma)-f(\zeta)) J_{\varepsilon}(\sigma-\zeta) \mathrm{d} \sigma,
$$

for all $u, \zeta \in \mathbb{R}$. Furthermore, if $f$ is Lipschitz continuous, then

$$
\int_{\mathbb{R}}\left|R_{\varepsilon}^{f}(u, \zeta)\right| \mathrm{d} \zeta \leq \varepsilon\|f\|_{\text {Lip }}|u|
$$

Suppose $A^{\prime} \geq \eta>0$ and let $g$ be defined by $g \circ A=f$. For $a, b \in \mathbb{R}$, let

$$
Z(a, b)=\int_{\mathbb{R}} \operatorname{sign}_{\varepsilon}(\zeta-a) R_{\varepsilon}^{g^{\prime}}(b ; \zeta) \mathrm{d} \zeta .
$$

Then

$$
|Z(a, b)| \leq 4\|f\|_{\text {Lip }} \frac{\varepsilon}{\eta}
$$

Proof. Observe that

$$
\int_{\mathbb{R}} f(\sigma) \chi(u ; \sigma) J_{\varepsilon}(\zeta-\sigma) \mathrm{d} \sigma=\underbrace{\int_{\mathbb{R}}(f(\sigma)-f(\zeta)) \chi(u ; \sigma) J_{\varepsilon}(\zeta-\sigma) \mathrm{d} \sigma}_{R_{\varepsilon}^{f}(u, \zeta)}+f(\zeta) \chi_{\varepsilon}(u ; \zeta) .
$$

Let $q^{\prime}(\sigma)=(f(\sigma)-f(\zeta)) J_{\varepsilon}(\zeta-\sigma)$. Equation (5.18) follows, since

$$
\int_{\mathbb{R}}(f(\sigma)-f(\zeta)) \chi(u ; \sigma) J_{\varepsilon}(\zeta-\sigma) \mathrm{d} \sigma=q(u)-q(0)=\int_{0}^{u} q^{\prime}(\sigma) \mathrm{d} \sigma .
$$

To prove (5.19) observe that

$$
\int_{\mathbb{R}}\left|R_{\varepsilon}^{f}(u, \zeta)\right| \mathrm{d} \zeta \leq \int_{\mathbb{R}}|\chi(u ; \sigma)|\left(\int_{\mathbb{R}}|f(\sigma)-f(\zeta)| J_{\varepsilon}(\zeta-\sigma) \mathrm{d} \zeta\right) \mathrm{d} \sigma .
$$

The result follows as

$$
\int_{\mathbb{R}}|f(\sigma)-f(\zeta)| J_{\varepsilon}(\zeta-\sigma) \mathrm{d} \sigma \leq\|f\|_{\text {Lip }} \varepsilon
$$

Let us prove (5.20). Take

$$
H_{\varepsilon}^{g^{\prime}}(b ; \zeta)=\int_{0}^{\zeta} R_{\varepsilon}^{g^{\prime}}(b ; \sigma) \mathrm{d} \sigma
$$

Integration by parts yields

$$
Z(a, b)=-2 \int_{\mathbb{R}} J_{\varepsilon}(\zeta-a) H_{\varepsilon}^{g^{\prime}}(b ; \zeta) \mathrm{d} \zeta=-\left(H_{\varepsilon}^{g^{\prime}}(b ; \cdot) \star J_{\varepsilon}\right)(a) .
$$

By (5.18),

$$
H_{\varepsilon}^{g^{\prime}}(b ; a)=\int_{0}^{a} \int_{0}^{b}\left(g^{\prime}(\omega)-g^{\prime}(\sigma)\right) J_{\varepsilon}(\omega-\sigma) \mathrm{d} \omega \mathrm{d} \sigma .
$$


Due to the symmetry of $J_{\varepsilon}$,

$$
\begin{aligned}
H_{\varepsilon}^{g^{\prime}}(b ; a)= & \int_{0}^{a} \int_{0}^{b} g^{\prime}(\omega) J_{\varepsilon}(\omega-\sigma) \mathrm{d} \omega \mathrm{d} \sigma \\
& -\int_{0}^{a} \int_{0}^{b} g^{\prime}(\sigma) J_{\varepsilon}(\omega-\sigma) \mathrm{d} \omega \mathrm{d} \sigma \\
= & \int_{0}^{a} \int_{0}^{b} g^{\prime}(\omega) J_{\varepsilon}(\omega-\sigma) \mathrm{d} \omega \mathrm{d} \sigma \\
& -\int_{0}^{b} \int_{0}^{a} g^{\prime}(\omega) J_{\varepsilon}(\omega-\sigma) \mathrm{d} \omega \mathrm{d} \sigma \\
= & \int_{\mathbb{R}} g^{\prime}(\omega) \chi(b ; \omega)\left(\int_{0}^{a} J_{\varepsilon}(\omega-\sigma) \mathrm{d} \sigma\right) \mathrm{d} \omega \\
& -\int_{\mathbb{R}} g^{\prime}(\omega) \chi(a ; \omega)\left(\int_{0}^{b} J_{\varepsilon}(\omega-\sigma) \mathrm{d} \sigma\right) \mathrm{d} \omega .
\end{aligned}
$$

Note that

$$
\int_{0}^{a} J_{\varepsilon}(\omega-\sigma) \mathrm{d} \sigma=\int_{\mathbb{R}} \chi(a ; \sigma) J_{\varepsilon}(\omega-\sigma) \mathrm{d} \sigma=\chi_{\varepsilon}(a ; \omega) .
$$

Hence,

$$
H_{\varepsilon}^{g^{\prime}}(b ; a)=\int_{\mathbb{R}} g^{\prime}(\omega)\left(\chi(b ; \omega) \chi_{\varepsilon}(a ; \omega)-\chi(a ; \omega) \chi_{\varepsilon}(b ; \omega)\right) \mathrm{d} \omega .
$$

Set

$$
\lambda(a, b ; \omega):=\chi(b ; \omega) \chi_{\varepsilon}(a ; \omega)-\chi(a ; \omega) \chi_{\varepsilon}(b ; \omega) .
$$

To find the support of $\lambda(a, b ; \omega)$ we first observe that $\lambda(a, b ; \omega)=-\lambda(b, a ; \omega)$. This reduces the situation to the following cases:

$$
\begin{cases}0 \leq a \leq b: & |\lambda(a, b ; \omega)| \leq \mathbb{1}_{|a-\omega| \leq \varepsilon} \\ b \leq a \leq 0: & |\lambda(a, b ; \omega)| \leq \mathbb{1}_{|a-\omega| \leq \varepsilon} \\ a \leq 0 \leq b: & |\lambda(a, b ; \omega)| \leq \mathbb{1}_{|\omega| \leq \varepsilon}\end{cases}
$$

It thus follows that

$$
\left|H_{\varepsilon}^{g^{\prime}}(b, a)\right| \leq 2\left\|g^{\prime}\right\|_{\infty} \varepsilon
$$

Statement (5.20) follows as $g^{\prime}(A(z)) A^{\prime}(z)=f^{\prime}(z)$, which implies $\left\|g^{\prime}\right\|_{\infty} \leq\|f\|_{\text {Lip }} \eta^{-1}$.

We have now the tools needed to start estimating the error terms in Lemmas 5.1 and 5.4, starting with those in Lemma 5.4.

Estimate 5.8. Let $R_{1}^{ \pm}$be defined in Lemma 5.4. Then there exists a constant $C=C(d, J)$ such that

$$
\left\|\int_{\mathbb{R}} R_{1}^{+}(\zeta)+R_{1}^{-}(\zeta) \mathrm{d} \zeta\right\|_{L^{1}\left(\Pi_{T}^{r_{0}}\right)} \leq C \frac{\Delta x}{r^{2}}\left(1+\frac{\Delta x}{r}\right)\left\|D_{-} A\left(u_{\Delta x}\right)\right\|_{L^{1}\left(\Pi_{T} ; \mathbb{R}^{d}\right)} .
$$

Proof. Let us first make an observation regarding the similarity of these terms. By statement (iv) of Lemma 5.2, recalling also the definition of $\theta_{\Delta x, i}^{ \pm}$and $\tau_{\Delta x, i}^{ \pm}$in Lemma 5.3,

$$
\begin{aligned}
& S_{\Delta x_{i}}\left(J_{\varepsilon}\left(\zeta-\tau_{\Delta x, i}^{-}\right)-J_{\varepsilon}\left(\zeta-\theta_{\Delta x, i}^{-}\right)\right) \\
& \quad=J_{\varepsilon}\left(\zeta-\tau_{\varepsilon}\left(S_{\Delta x_{i}} A\left(u_{\Delta x}\right), A\left(u_{\Delta x}\right), \zeta\right)\right)-J_{\varepsilon}\left(\zeta-\theta_{\varepsilon}\left(S_{\Delta x_{i}} A\left(u_{\Delta x}\right), A\left(u_{\Delta x}\right), \zeta\right)\right) \\
& \quad=-\left(J_{\varepsilon}\left(\zeta-\tau_{\Delta x, i}^{+}\right)-J_{\varepsilon}\left(\zeta-\theta_{\Delta x, i}^{+}\right)\right) .
\end{aligned}
$$


Recalling that $\rho_{\varepsilon, r, r_{0}}=\chi_{\varepsilon}(A(u) ; \cdot) \star J_{r_{0}} \otimes J_{r}$, which implies

$$
\begin{aligned}
R_{1}^{+}(\zeta)+R_{1}^{-}(\zeta)=- & \sum_{i=1}^{d} S_{\Delta x_{i}}\left[\left(J_{\varepsilon}\left(\zeta-\tau_{\Delta x, i}^{-}\right)-J_{\varepsilon}\left(\zeta-\theta_{\Delta x, i}^{-}\right)\right) D_{-}^{i}\left(u_{\Delta x}\right) \star\left(J_{r_{0}} \otimes J_{r}\right)\right] \partial_{x_{i}} \rho_{\varepsilon, r, r_{0}} \\
& +\sum_{i=1}^{d}\left[\left(J_{\varepsilon}\left(\zeta-\tau_{\Delta x, i}^{-}\right)-J_{\varepsilon}\left(\zeta-\theta_{\Delta x, i}^{-}\right)\right) D_{-}^{i}\left(u_{\Delta x}\right) \star\left(J_{r_{0}} \otimes J_{r}\right)\right] \partial_{x_{i}} \rho_{\varepsilon, r, r_{0}} \\
= & -\sum_{i=1}^{d}\left(S_{\Delta x_{i}}-1\right) \partial_{x_{i}} \rho_{\varepsilon, r, r_{0}}\left[\left(J_{\varepsilon}\left(\zeta-\tau_{\Delta x, i}^{-}\right)-J_{\varepsilon}\left(\zeta-\theta_{\Delta x, i}^{-}\right)\right) D_{-}^{i}\left(u_{\Delta x}\right) \star\left(J_{r_{0}} \otimes J_{r}\right)\right] \\
= & -\Delta x \sum_{i=1}^{d}\left({ }_{{ }_{\varepsilon}\left(\zeta, u_{\Delta x}\right)}^{\left.\left.\left(\zeta-\tau_{\Delta x, i}^{-}\right)-J_{\varepsilon}\left(\zeta-\theta_{\Delta x, i}^{-}\right)\right) D_{-}^{i} A\left(u_{\Delta x}\right)\right) \chi_{\varepsilon}(A(u) ; \zeta)}\left(J_{r_{0}} \otimes \partial_{x_{i}} J_{r}\right) \otimes\left(J_{r_{0}} \otimes D_{+}^{i} J_{r}\right) .\right.
\end{aligned}
$$

By statement (ii) of Lemma 5.6,

$$
\left|\int_{\mathbb{R}} R_{1}^{+}(\zeta)+R_{1}^{-}(\zeta) \mathrm{d} \zeta\right| \leq \frac{\Delta x}{2} \sum_{i=1}^{d}\left\|\chi_{\varepsilon}(A(u) ; \cdot)\right\|_{L^{\infty}(\mathbb{R})}\left|D_{-}^{i} A\left(u_{\Delta x}\right)\right|^{\left(u, u_{\Delta x}\right)}\left|J_{r_{0}} \otimes \partial_{x_{i}} J_{r} \otimes J_{r_{0}} \otimes D_{+}^{i} J_{r}\right| .
$$

By Lemma 5.5,

$$
\left\|\int_{\mathbb{R}} R_{1}^{+}(\zeta)+R_{1}^{-}(\zeta) \mathrm{d} \zeta\right\|_{L^{1}\left(\Pi_{T}^{\left.r_{0}\right)}\right.} \leq \frac{\Delta x}{2} \sum_{i=1}^{d}\left\|D_{-}^{i} A\left(u_{\Delta x}\right)\right\|_{L^{1}\left(\Pi_{T}\right)} \times\left\|\partial_{x_{i}} J_{r}\right\|_{L^{1}\left(\mathbb{R}^{d}\right)}\left\|D_{+}^{i} J_{r}\right\|_{L^{1}\left(\mathbb{R}^{d}\right)} .
$$

Recall that $\left\|\partial_{x_{i}} J_{r}\right\|_{L^{1}\left(\mathbb{R}^{d}\right)} \leq 2\left\|J^{\prime}\right\|_{\infty} r^{-1}$. Note that

$$
\left|D_{+}^{i} J_{r}(x)\right|=\frac{1}{\Delta x}\left|J_{r}\left(x_{i}+\Delta x\right)-J_{r}\left(x_{i}\right)\right| \prod_{j \neq i} J_{r}\left(x_{j}\right) \leq \frac{1}{r^{2}}\left\|J^{\prime}\right\|_{\infty} \mathbb{1}_{\left|x_{i}\right| \leq r+\Delta x} \prod_{j \neq i} J_{r}\left(x_{j}\right) .
$$

Hence

$$
\left\|D_{+}^{i} J_{r}\right\|_{L^{1}\left(\mathbb{R}^{d}\right)} \leq \frac{1}{r^{2}}\left\|J^{\prime}\right\|_{\infty} \int_{\mathbb{R}} \mathbb{1}_{\left|x_{i}\right| \leq r+\Delta x} \mathrm{~d} x_{i}=2\left\|J^{\prime}\right\|_{\infty} \frac{1}{r}\left(1+\frac{\Delta x}{r}\right)
$$

The estimate follows from (5.22) and (5.21).

Estimate 5.9. Let $R_{2}^{ \pm}$be defined in Lemma 5.4. Then there exists a constant $C=C(d, J)$ such that

$$
\left\|\int_{\mathbb{R}} R_{2}^{+}(\zeta)+R_{2}^{-}(\zeta) \mathrm{d} \zeta\right\|_{L^{1}\left(\Pi_{T}^{r_{0}}\right)} \leq C \frac{\Delta x}{\varepsilon^{2} \sqrt{r_{0} r^{d}}}\left\|D_{+} A\left(u_{\Delta x}\right)\right\|_{L^{2}\left(\Pi_{T} ; \mathbb{R}^{d}\right)}\|\nabla A(u)\|_{L^{2}\left(\Pi_{T} ; \mathbb{R}^{d}\right)}^{2} .
$$

Proof. Let us consider $R_{2}^{+}$. The term $R_{2}^{-}$is treated the same way. By Lemma 5.6,

$$
\begin{aligned}
\left|\int_{\mathbb{R}} R_{2}^{+}(\zeta) \mathrm{d} \zeta\right| \leq & \frac{1}{2} \sum_{i=1}^{d}\left|\int_{\mathbb{R}}\left(J_{\varepsilon}\left(\zeta-A\left(u_{\Delta x}\right)\right)-J_{\varepsilon}\left(\zeta-A\left(\tau_{\Delta x, i}^{+}\right)\right)\right) J_{\varepsilon}(\zeta-A(u)) \mathrm{d} \zeta\right| \\
& \times\left(\partial_{x_{i}} A(u)\right)^{2} \stackrel{\left(u, u_{\Delta x}\right)}{\star}\left(J_{r_{0}} \otimes J_{r}\right) \otimes\left(J_{r_{0}} \otimes J_{r}\right) \\
\leq & \frac{1}{2} \sum_{i=1}^{d}\left|\int_{A\left(u_{\Delta x}\right)}^{S_{\Delta x_{i}} A\left(u_{\Delta x}\right)}\right| \partial_{\xi}\left(J_{\varepsilon}(\cdot-A(u)) \star J_{\varepsilon}(\xi)\right)|\mathrm{d} \xi| \\
& \times\left(\partial_{x_{i}} A(u)\right)^{2} \stackrel{\left(u, u_{\Delta x}\right)}{\star}\left(J_{r_{0}} \otimes J_{r}\right) \otimes\left(J_{r_{0}} \otimes J_{r}\right) .
\end{aligned}
$$


By Young's inequality for convolutions,

$$
\left\|J_{\varepsilon}(\cdot-A(u)) \star J_{\varepsilon}^{\prime}\right\|_{L^{\infty}(\mathbb{R})} \leq\left\|J_{\varepsilon}(\cdot-A(u))\right\|_{L^{\infty}(\mathbb{R})}\left\|J_{\varepsilon}^{\prime}\right\|_{L^{1}(\mathbb{R})} \leq \frac{2}{\varepsilon^{2}}\|J\|_{\infty}\left\|J^{\prime}\right\|_{\infty} .
$$

Hence,

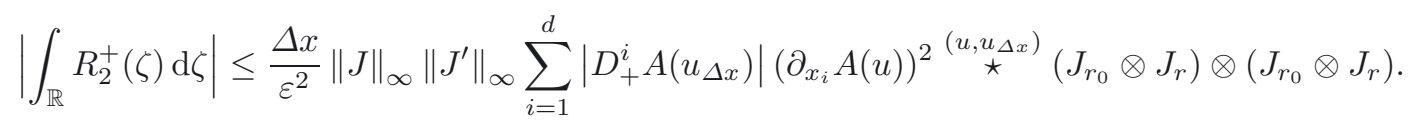

Applying Lemma 5.5, with $K_{1}(u)=|u|, K_{2}(v)=v^{2}, p=q=2$, we get

$$
\begin{aligned}
\left\|\int_{\mathbb{R}} R_{2}^{+}(\zeta) \mathrm{d} \zeta\right\|_{L^{1}\left(\Pi_{T}^{\left.r_{0}\right)}\right.} \leq \frac{\Delta x}{\varepsilon^{2}}\|J\|_{\infty}\left\|J^{\prime}\right\|_{\infty} \sum_{i=1}^{d}\left\|D_{+}^{i} A\left(u_{\Delta x}\right)\right\|_{L^{2}\left(\Pi_{T}\right)}\left\|\partial_{x_{i}} A(u)\right\|_{L^{2}\left(\Pi_{T}\right)}^{2} \\
\times\left\|J_{r_{0}} \otimes J_{r}\right\|_{L^{2}\left(\mathbb{R} \times \mathbb{R}^{d}\right)}\left\|J_{r_{0}} \otimes J_{r}\right\|_{L^{1}\left(\mathbb{R} \times \mathbb{R}^{d}\right)} .
\end{aligned}
$$

Now

$$
\left\|J_{r_{0}} \otimes J_{r}\right\|_{L^{2}\left(\mathbb{R} \times \mathbb{R}^{d}\right)}=\left\|J_{r_{0}}\right\|_{L^{2}(\mathbb{R})} \prod_{i=1}^{d}\left\|J_{r}\right\|_{L^{2}(\mathbb{R})}=\frac{1}{\sqrt{r_{0} r^{d}}}\|J\|_{L^{2}(\mathbb{R})}^{d+1} .
$$

Estimate 5.10. Let $R_{1}^{ \pm}$be defined in Lemma 5.4 and suppose $d=1$. Then there exists a constant $C=C(J)$ such that

$$
\left\|\lim _{\varepsilon \downarrow 0} \int_{\mathbb{R}} R_{2}^{+}(\zeta)+R_{2}^{-}(\zeta) \mathrm{d} \zeta\right\|_{L^{1}\left(\Pi_{T}^{r_{0}}\right)} \leq C\left(\frac{\Delta x}{r}\|f\|_{\text {Lip }}+\frac{\Delta x}{r^{2}}\|A\|_{\text {Lip }}+\frac{\Delta x}{r_{0}}\right)\left\|D_{+} u_{\Delta x}\right\|_{L^{1}\left(\Pi_{T}\right)} .
$$

Proof. We consider $R_{2}^{+}$. The $R_{2}^{-}$term can be treated similarly. Note that for $d=1$,

$$
\int_{\mathbb{R}} R_{2}^{+}(\zeta) \mathrm{d} \zeta=\int_{\mathbb{R}}\left(J_{\varepsilon}\left(\zeta-A\left(u_{\Delta x}\right)\right)-J_{\varepsilon}\left(\zeta-A\left(\tau_{\Delta x}^{+}\right)\right)\right) n_{A, \varepsilon, r, r_{0}}(\zeta) \mathrm{d} \zeta \stackrel{\left(u_{\star} x\right)}{\star} J_{r_{0}} \otimes J_{r},
$$

where $n_{A, \varepsilon, r, r_{0}}$ is defined in Lemma 4.7. The map $\zeta \mapsto n_{A, \varepsilon, r, r_{0}}(t, x, \zeta)$ belongs to $W_{\text {loc }}^{1,1}(\mathbb{R})$ for each fixed $(t, x) \in\left(r_{0}, T-r_{0}\right) \times \mathbb{R}$. Due to Lemmas $4.7,5.7$, and 4.6 ,

$$
\lim _{\varepsilon \rightarrow 0} \partial_{\zeta} n_{A, \varepsilon, r, r_{0}}(\zeta)=B^{\prime}(\zeta) \partial_{t} \rho_{r, r_{0}}(\zeta)+g^{\prime}(\zeta) \partial_{x} \rho_{r, r_{0}}(\zeta)-\partial_{x}^{2} \rho_{r, r_{0}}(\zeta)
$$

in $L^{1}(\mathbb{R})$ for each fixed $(t, x)$, where

$$
\rho_{r, r_{0}}(\zeta)=\chi(A(u) ; \zeta) \star J_{r_{0}} \otimes J_{r}
$$

By statement (iii) of Lemma 5.6,

$$
\begin{aligned}
\left|\lim _{\varepsilon \downarrow 0} \int_{\mathbb{R}} R_{2}^{+}(\zeta) \mathrm{d} \zeta\right| \leq & \left|\int_{A\left(u_{\Delta x}\right)}^{S_{\Delta x} A\left(u_{\Delta x}\right)}\right| B^{\prime}(\zeta) \partial_{t} \rho_{r, r_{0}}(\zeta)|\mathrm{d} \zeta|^{\left(u_{\Delta x}\right)} J_{r_{0}} \otimes J_{r} \\
& +\left|\int_{A\left(u_{\Delta x}\right)}^{S_{\Delta x} A\left(u_{\Delta x}\right)}\right| g^{\prime}(\zeta) \partial_{x} \rho_{r, r_{0}}(\zeta)|\mathrm{d} \zeta|^{\stackrel{\left(u_{\Delta x}\right)}{\star} J_{r_{0}} \otimes J_{r}} \\
& +\left|\int_{A\left(u_{\Delta x}\right)}^{S_{\Delta x} A\left(u_{\Delta x}\right)}\right| \partial_{x}^{2} \rho_{r, r_{0}}(\zeta)|\mathrm{d} \zeta|^{\left(u_{\Delta x}\right)} J_{r_{0}} \otimes J_{r} \\
= & \mathscr{T}_{1}+\mathscr{T}_{2}+\mathscr{T}_{3} .
\end{aligned}
$$


We consider each term separately. Let $B(\zeta)=\xi$ or equivalently $A(\xi)=\zeta$. It follows that

$$
\begin{aligned}
\mathscr{T}_{1} & \leq\left|\int_{A(u \Delta x)}^{S_{\Delta x} A(u \Delta x)}\right| B^{\prime}(\zeta) \chi(A(u) ; \zeta)|\mathrm{d} \zeta|^{\left(u, u_{\Delta x}\right)}\left|\partial_{t} J_{r_{0}}\right| \otimes J_{r} \otimes J_{r_{0}} \otimes J_{r} \\
& =\left|\int_{u_{\Delta x}}^{S_{\Delta x} u \Delta x}\right| \chi(u ; \xi)|\mathrm{d} \xi|^{\left(u, u_{\Delta x}\right)}\left|\partial_{t} J_{r_{0}}\right| \otimes J_{r} \otimes J_{r_{0}} \otimes J_{r} \\
& \leq \Delta x\left|D_{+} A\left(u_{\Delta x}\right)\right|_{\star}^{\left(u, u_{\Delta x}\right)}\left|\partial_{t} J_{r_{0}}\right| \otimes J_{r} \otimes J_{r_{0}} \otimes J_{r} .
\end{aligned}
$$

By Lemma 5.5,

$$
\left\|\mathscr{T}_{1}\right\|_{L^{1}\left(\Pi_{T}^{r_{0}}\right)} \leq 2 \frac{\Delta x}{r_{0}}\left\|J^{\prime}\right\|_{\infty}\left\|D_{+} u_{\Delta x}\right\|_{L^{1}\left(\Pi_{T}\right)} .
$$

Observe that $g^{\prime}(A(\xi)) A^{\prime}(\xi)=f^{\prime}(\xi)$ and $\mathrm{d} \zeta=A^{\prime}(\xi) \mathrm{d} \xi$. Hence,

$$
\begin{aligned}
\mathscr{T}_{2} & \leq\left|\int_{A\left(u_{\Delta x}\right)}^{S_{\Delta x} A\left(u_{\Delta x}\right)}\right| g^{\prime}(\zeta) \chi(A(u) ; \zeta)|\mathrm{d} \zeta|^{\left(u, u_{\Delta x}\right)} J_{r_{0}} \otimes\left|\partial_{x} J_{r}\right| \otimes J_{r_{0}} \otimes J_{r} \\
& =\left|\int_{u_{\Delta x}}^{S_{\Delta x} u_{\Delta x}}\right| f^{\prime}(\xi) \chi(u ; \xi)|\mathrm{d} \xi|^{\left(u, u_{\Delta x}\right)} J_{r_{0}} \otimes\left|\partial_{x} J_{r}\right| \otimes J_{r_{0}} \otimes J_{r} \\
& \leq \Delta x\|f\|_{\text {Lip }}\left|D_{+} u_{\Delta x}\right|_{\stackrel{\left(u, u_{\Delta x}\right)}{\star} J_{r_{0}} \otimes\left|\partial_{x} J_{r}\right| \otimes J_{r_{0}} \otimes J_{r} .}
\end{aligned}
$$

By Lemma 5.5,

$$
\left\|\mathscr{T}_{2}\right\|_{L^{1}\left(\Pi_{T}^{r_{0}}\right)} \leq 2\|f\|_{\text {Lip }} \frac{\Delta x}{r}\left\|J^{\prime}\right\|_{\infty}\left\|D_{+} u_{\Delta x}\right\|_{L^{1}\left(\Pi_{T}\right)}
$$

Similarly,

$$
\begin{aligned}
\mathscr{T}_{3} & \leq\left|\int_{A\left(u_{\Delta x}\right)}^{S_{\Delta x} A\left(u_{\Delta x}\right)}\right| \chi(A(u) ; \zeta)|\mathrm{d} \zeta| \stackrel{\left(u, u_{\Delta x}\right)}{\star} J_{r_{0}} \otimes\left|\partial_{x}^{2} J_{r}\right| \otimes J_{r_{0}} \otimes J_{r} \\
& \leq \Delta x\left|D_{+} A\left(u_{\Delta x}\right)\right| \stackrel{\left(u, u_{\Delta x}\right)}{\star} J_{r_{0}} \otimes\left|\partial_{x}^{2} J_{r}\right| \otimes J_{r_{0}} \otimes J_{r} .
\end{aligned}
$$

By Lemma 5.5,

$$
\left\|\mathscr{T}_{3}\right\|_{L^{1}\left(\Pi_{T}^{r_{0}}\right)} \leq 2 \frac{\Delta x}{r^{2}}\left\|J^{\prime \prime}\right\|_{\infty}\left\|D_{+} A\left(u_{\Delta x}\right)\right\|_{L^{1}\left(\Pi_{T}\right)} .
$$

Estimate 5.11. Let $U$ be the second term in (5.3), Lemma 5.1, that is,

$$
U=2 \int_{\mathbb{R}} \nabla \rho_{\varepsilon, r, r_{0}} \cdot\left(2 \nabla-\left(D_{+}+D_{-}\right) \rho_{\varepsilon, r, r_{0}}^{\Delta x} \mathrm{~d} \zeta .\right.
$$

Then there exists a constant $C=C(d, J)$ such that

$$
\|U\|_{L^{1}\left(\Pi_{T}^{r_{0}}\right)} \leq C \frac{\Delta x^{2}}{r^{3}}\left(1+\frac{\Delta x}{r}\right)\left\|A\left(u_{\Delta x}\right)\right\|_{L^{1}\left([0, T] ; B V\left(\mathbb{R}^{d}\right)\right)} .
$$

Remark 5.12. The $B V$ norm may be replaced by the $L^{1}$ norm at the expense of an extra factor $r^{-1}$.

Proof. Clearly,

$$
\begin{aligned}
& \| \int_{\mathbb{R}} \nabla \rho_{\varepsilon, r, r_{0}} \cdot\left(2 \nabla-\left(D_{+}+D_{-}\right) \rho_{\varepsilon, r, r_{0}}^{\Delta x} \mathrm{~d} \zeta \|_{L^{1}\left(\Pi_{T}^{r_{0}}\right)}\right. \\
& \leq\left\|\nabla \rho_{\varepsilon, r, r_{0}}\right\|_{L^{\infty}\left(\Pi_{T}^{r_{0}} \times \mathbb{R} ; \mathbb{R}_{\infty}^{d}\right)} \|\left(2 \nabla-\left(D_{+}+D_{-}\right) \rho_{\varepsilon, r, r_{0}}^{\Delta x} \|_{L^{1}\left(\Pi_{T}^{r_{0}} \times \mathbb{R} ; \mathbb{R}_{1}^{d}\right)} .\right.
\end{aligned}
$$


By Young's inequality for convolutions,

$$
\begin{aligned}
\left\|\partial_{x_{i}} \rho_{\varepsilon, r, r_{0}}\right\|_{L^{\infty}\left(\Pi_{T}^{r_{0}} \times \mathbb{R}\right)} & \leq\|\chi(A(u) ; \cdot)\|_{L^{\infty}\left(\Pi_{T} \times \mathbb{R}\right)}\left\|J_{\varepsilon} \otimes \partial_{x_{i}} J_{r} \otimes J_{r_{0}}\right\|_{L^{1}\left(\mathbb{R} \times \mathbb{R}^{d} \times \mathbb{R}\right)} \\
& \leq\left\|\partial_{x_{i}} J_{r}\right\|_{L^{1}\left(\mathbb{R}^{d}\right)} \leq 2\left\|J^{\prime}\right\|_{\infty} r^{-1}
\end{aligned}
$$

We have

$$
\left(2 \partial_{x_{i}}-\left(D_{+}^{i}+D_{-}^{i}\right)\right) \rho_{\varepsilon, r, r_{0}}^{\Delta x}=\chi\left(A\left(u_{\Delta x}\right) ; \cdot\right) \star J_{\varepsilon} \otimes\left(2 \partial_{x_{i}}-\left(D_{+}^{i}+D_{-}^{i}\right)\right) J_{r} \otimes J_{r_{0}} .
$$

Using Taylor expansions with remainders,

$$
\begin{aligned}
\left(\left(D_{+}^{i}+D_{-}^{i}\right)-2 \partial_{x_{i}}\right) J_{r}(x)= & \frac{1}{2 \Delta x} \int_{0}^{\Delta x}(z-\Delta x)^{2} \partial_{x_{i}}^{3} J_{r}\left(x_{i}+z\right) \mathrm{d} z \prod_{j \neq i} J_{r}\left(x_{j}\right) \\
& +\frac{1}{2 \Delta x} \int_{-\Delta x}^{0}(z+\Delta x)^{2} \partial_{x_{i}}^{3} J_{r}\left(x_{i}+z\right) \mathrm{d} z \prod_{j \neq i} J_{r}\left(x_{j}\right) \\
= & : \partial_{x_{i}}\left(\varphi_{1}^{i}(x)+\varphi_{2}^{i}(x)\right),
\end{aligned}
$$

see for instance ([25], p. 25). Hence

$$
\left(2 \partial_{x_{i}}-\left(D_{+}^{i}+D_{-}^{i}\right)\right) \rho_{\varepsilon, r, r_{0}}^{\Delta x}=\partial_{x_{i}}\left(\chi\left(A\left(u_{\Delta x}\right) ; \cdot\right) \star J_{\varepsilon} \otimes\left(\varphi_{1}^{i}+\varphi_{2}^{i}\right) \otimes J_{r_{0}}\right) .
$$

By Young's inequality for convolutions

$$
\left\|\left(2 \partial_{x_{i}}-\left(D_{+}^{i}+D_{-}^{i}\right)\right) \rho_{\varepsilon, r, r_{0}}^{\Delta x}\right\|_{L^{1}\left(\Pi_{T}^{r_{0}} \times \mathbb{R}\right)} \leq\left\|\chi\left(A\left(u_{\Delta x}\right) ; \cdot\right)\right\|_{L^{1}\left([0, T] \times \mathbb{R} ; B V\left(\mathbb{R}^{d}\right)\right)} \times\left\|\varphi_{1}^{i}+\varphi_{2}^{i}\right\|_{L^{1}\left(\mathbb{R}^{d}\right)} .
$$

Note that $\left\|\chi\left(A\left(u_{\Delta x}\right) ; \cdot\right)\right\|_{L^{1}\left([0, T] \times \mathbb{R} ; B V\left(\mathbb{R}^{d}\right)\right)}=\left\|A\left(u_{\Delta x}\right)\right\|_{L^{1}\left([0, T] ; B V\left(\mathbb{R}^{d}\right)\right)}$. Now, as $\partial_{x_{i}} J_{r}\left(x_{i}+z\right) \leq r^{-3}\left\|J^{\prime \prime}\right\|_{\infty} \mathbb{1}_{\left|x_{i}+z\right| \leq r}$, it follows that

$$
\begin{aligned}
\left\|\varphi_{1}^{i}\right\|_{L^{1}\left(\mathbb{R}^{d}\right)} & =\frac{1}{2 \Delta x} \int_{\mathbb{R}}\left|\int_{0}^{\Delta x}(z-\Delta x)^{2} \partial_{x_{i}}^{2} J_{r}\left(x_{i}+z\right) \mathrm{d} z\right| \mathrm{d} x_{i} \\
& \leq \frac{(r+\Delta x)}{\Delta x r^{3}}\left\|J^{\prime \prime}\right\|_{\infty} \int_{0}^{\Delta x}(z-\Delta x)^{2} \mathrm{~d} z \\
& \leq \frac{1}{3}\left\|J^{\prime \prime}\right\|_{\infty} \frac{\Delta x^{2}}{r^{2}}\left(1+\frac{\Delta x}{r}\right) .
\end{aligned}
$$

The same estimate applies to $\varphi_{2}^{i}$.

Estimate 5.13. Let $\mathscr{T}$ be the term (5.9) from Lemma 5.1, that is,

$$
\mathscr{T}=\int_{R}\left(\operatorname{sign}_{\varepsilon}(\zeta-A(u)) \star J_{r_{0}} \otimes J_{r}\right)\left(\Delta-D_{-} \cdot D_{+}\right) \rho_{\varepsilon, r, r_{0}}^{\Delta x} \mathrm{~d} \zeta .
$$

Then there exists a constant $C=C(d, J)$ such that

$$
\|\mathscr{T}\|_{L^{1}\left(\Pi_{T}^{r_{0}}\right)} \leq C \frac{\Delta x^{2}}{r^{3}}\left(1+\frac{\Delta x}{r}\right)\left\|A\left(u_{\Delta x}\right)\right\|_{L^{1}\left([0, T] ; B V\left(\mathbb{R}^{d}\right)\right)} .
$$

Remark 5.14. At the cost of an extra factor $r^{-1}$, the $B V$ norm may be replaced by the $L^{1}$ norm. 
Proof. First note that $\left|\operatorname{sign}_{\varepsilon}(\zeta-A(u)) \star J_{r_{0}} \otimes J_{r}\right| \leq 1$, so

$$
|\mathscr{T}| \leq\left\|\left(\Delta-D_{-} \cdot D_{+}\right) \rho_{\varepsilon, r, r_{0}}^{\Delta x}\right\|_{L^{1}\left(\Pi_{T} \times \mathbb{R}\right)} .
$$

Now,

$$
\left(\partial_{x_{i}}^{2}-D_{-}^{i} D_{+}^{i}\right) \rho_{\varepsilon, r, r_{0}}^{\Delta x}=\chi\left(A\left(u_{\Delta x}\right) ; \cdot\right) \star J_{\varepsilon} \otimes\left(\partial_{x_{i}}^{2}-D_{-}^{i} D_{+}^{i}\right) J_{r} \otimes J_{r_{0}} .
$$

Using a Taylor expansion ([25], p. 24),

$$
\begin{aligned}
\left(\partial_{x_{i}}^{2}-D_{-}^{i} D_{+}^{i}\right) J_{r}(x)= & \frac{1}{6 \Delta x^{2}} \int_{0}^{\Delta x}(z-\Delta x)^{3} \partial_{x_{i}}^{4} J_{r}\left(x_{i}+z\right) \mathrm{d} z \prod_{j \neq i} J_{r}\left(x_{j}\right) \\
& -\frac{1}{6 \Delta x^{2}} \int_{-\Delta x}^{0}(z+\Delta x)^{3} \partial_{x_{i}}^{4} J_{r}\left(x_{i}+z\right) \mathrm{d} z \prod_{j \neq i} J_{r}\left(x_{j}\right) \\
= & : \partial_{x_{i}}\left(\varphi_{1}^{i}(x)+\varphi_{2}^{i}(x)\right) .
\end{aligned}
$$

Hence,

$$
\left(\partial_{x_{i}}^{2}-D_{-}^{i} D_{+}^{i}\right) \rho_{\varepsilon, r, r_{0}}^{\Delta x}=\partial_{x_{i}}\left(\chi\left(A\left(u_{\Delta x}\right) ; \cdot\right) \star J_{\varepsilon} \otimes\left(\varphi_{1}^{i}+\varphi_{2}^{i}\right) \otimes J_{r_{0}}\right) .
$$

By Young's inequality for convolutions,

$$
\left\|\left(\partial_{x_{i}}^{2}-D_{-}^{i} D_{+}^{i}\right) \rho_{\varepsilon, r, r_{0}}^{\Delta x}\right\|_{L^{1}\left(\Pi_{T} \times \mathbb{R}\right)} \leq\left\|\chi\left(A\left(u_{\Delta x}\right) ; \cdot\right)\right\|_{L^{1}\left([0, T] \times \mathbb{R} ; B V\left(\mathbb{R}^{d}\right)\right)} \times\left\|\varphi_{1}^{i}+\varphi_{2}^{i}\right\|_{L^{1}\left(\mathbb{R}^{d}\right)} .
$$

It remains to estimate the $L^{1}$ norm of $\varphi_{1}^{i}$ and $\varphi_{2}^{i}$ :

$$
\begin{aligned}
\left\|\varphi_{1}^{i}\right\|_{L^{1}\left(\mathbb{R}^{d}\right)} & =\frac{1}{6 \Delta x^{2}} \int_{\mathbb{R}}\left|\int_{0}^{\Delta x}(z-\Delta x)^{3} \partial_{x_{i}}^{3} J_{r}\left(x_{i}+z\right) \mathrm{d} z\right| \mathrm{d} x_{i} \\
& \leq \frac{r+\Delta x}{3 \Delta x^{2} r^{4}}\left\|J^{(3)}\right\|_{\infty}\left|\int_{0}^{\Delta x}(z-\Delta x)^{3} \mathrm{~d} z\right| \\
& =\frac{\left\|J^{(3)}\right\|_{\infty}}{12} \frac{\Delta x^{2}}{r^{3}}\left(1+\frac{\Delta x}{r}\right) .
\end{aligned}
$$

A similar estimate applies to $\varphi_{2}^{i}$.

Estimate 5.15. Let $\mathscr{T}_{1}$ and $\mathscr{T}_{2}$ be the terms from (5.8) in Lemma 5.1, that is,

$$
\begin{aligned}
& \mathscr{T}_{1}=\int_{\mathbb{R}}\left(\operatorname{sign}_{\varepsilon}(\zeta-A(u)) \star J_{r_{0}} \otimes J_{r}\right) G_{1}^{\prime}(\zeta) \cdot\left(D_{+}-\nabla\right) \rho_{\varepsilon, r, r_{0}}^{\Delta x} \mathrm{~d} \zeta, \\
& \mathscr{T}_{2}=\int_{\mathbb{R}}\left(\operatorname{sign}_{\varepsilon}(\zeta-A(u)) \star J_{r_{0}} \otimes J_{r}\right) G_{2}^{\prime}(\zeta) \cdot\left(D_{-}-\nabla\right) \rho_{\varepsilon, r, r_{0}}^{\Delta x} \mathrm{~d} \zeta,
\end{aligned}
$$

where $G_{k}(A(u))=F_{k}(u)$ for $j=1,2$. Then there exists a constant $C=C(d, J)$ such that

$$
\left\|\mathscr{T}_{k}\right\|_{L^{1}\left(\Pi_{T}^{r_{0}}\right)} \leq C \frac{\Delta x}{r}\left(1+\frac{\Delta x}{r}\right)\left\|F_{k}^{\prime}\right\|_{L^{\infty}\left(\mathbb{R} ; \mathbb{R}^{d}\right)}\left\|A\left(u_{\Delta x}\right)\right\|_{L^{1}\left([0, T] ; B V\left(\mathbb{R}^{d}\right)\right)}
$$

for $k=1,2$. 
Remark 5.16. Again the $B V$ norm may be replaced by the $L^{1}$ norm at the cost of an extra factor $r^{-1}$.

Proof. Consider $\mathscr{T}_{1}$. We can change variables $\zeta=A(\xi)$, which yields

$$
\mathscr{T}_{1}=\int_{\mathbb{R}}\left(\operatorname{sign}_{\varepsilon}(A(\xi)-A(u)) \star J_{r_{0}} \otimes J_{r}\right) F_{1}^{\prime}(\zeta) \cdot\left(D_{+}-\nabla\right) \rho_{\varepsilon, r, r_{0}}^{\Delta x} \mathrm{~d} \xi .
$$

Then observe that

$$
\left\|\mathscr{T}_{1}\right\|_{L^{1}\left(\Pi_{T}^{r_{0}}\right)} \leq\left\|F_{1}^{\prime} \cdot\left(D_{+}-\nabla\right)\left(\chi\left(A\left(u_{\Delta x}\right) ; \cdot\right) \star J_{\varepsilon} \otimes J_{r} \otimes J_{r_{0}}\right)\right\|_{L^{1}\left(\Pi_{T} \times \mathbb{R}\right)} .
$$

We have

$$
\left(D_{+}^{i}-\partial_{x_{i}}\right)\left(\chi\left(A\left(u_{\Delta x}\right) ; \cdot\right) \star J_{\varepsilon} \otimes J_{r} \otimes J_{r_{0}}\right)=\chi\left(A\left(u_{\Delta x}\right) ; \cdot\right) \star J_{\varepsilon} \otimes\left(D_{+}^{i}-\partial_{x_{i}}\right) J_{r} \otimes J_{r_{0}} .
$$

By Taylor expansions,

$$
\begin{aligned}
\left(D_{+}^{i}-\partial_{x_{i}}\right) J_{r}(x) & =\frac{1}{\Delta x} \int_{0}^{\Delta x}(\Delta x-z) \partial_{x_{i}}^{2} J_{r}\left(x_{i}+z\right) \mathrm{d} z \prod_{j \neq i} J_{r}\left(x_{j}\right) \\
& =: \partial_{x_{i}} \varphi(x) .
\end{aligned}
$$

By Young's inequality for convolutions,

$$
\begin{aligned}
\left\|\left(F_{1}^{i}\right)^{\prime}\left(D_{+}^{i}-\partial_{x_{i}}\right)\left(\chi\left(A\left(u_{\Delta x}\right) ; \cdot\right) \star J_{\varepsilon} \otimes J_{r} \otimes J_{r_{0}}\right)\right\|_{L^{1}\left(\Pi_{T} \times \mathbb{R}\right)} & \\
& \leq\left\|\left(F_{1}^{i}\right)^{\prime}\right\|_{L^{\infty}(\mathbb{R})}\left\|\chi\left(A\left(u_{\Delta x}\right) ; \cdot\right)\right\|_{L^{1}\left([0, T] \times \mathbb{R} ; B V\left(\mathbb{R}^{d}\right)\right)}\|\varphi\|_{L^{1}\left(\mathbb{R}^{d}\right)}
\end{aligned}
$$

It remains to estimate $\|\varphi\|_{L^{1}\left(\mathbb{R}^{d}\right)}$ :

$$
\begin{aligned}
\|\varphi\|_{L^{1}\left(\mathbb{R}^{d}\right)} & =\frac{1}{\Delta x} \int_{\mathbb{R}}\left|\int_{0}^{\Delta x}(\Delta x-z) \partial_{x_{i}} J_{r}\left(x_{i}+z\right) \mathrm{d} z\right| \mathrm{d} x_{i} \\
& \leq \frac{1}{\Delta x} 2\left\|J^{\prime}\right\|_{\infty} \frac{r+\Delta x}{r^{2}} \int_{0}^{\Delta x}(\Delta x-z) \mathrm{d} z \\
& =\left\|J^{\prime}\right\|_{\infty} \frac{\Delta x}{r}\left(1+\frac{\Delta x}{r}\right)
\end{aligned}
$$

from which the estimate of $\mathscr{T}_{1}$ follows. Similar arguments apply to $\mathscr{T}_{2}$.

Estimate 5.17. Consider the terms (5.4), (5.5), (5.6), and (5.7) from Lemma 5.1. Suppose $A^{\prime}>\eta$ and set $B:=A^{-1}$. Let

$$
\begin{aligned}
\mathscr{T}_{1}= & \int_{\mathbb{R}}\left(\operatorname{sign}_{\varepsilon}\left(\zeta-A\left(u_{\Delta x}\right)\right) \star J_{r_{0}} \otimes J_{r}\right) \partial_{t} R_{\varepsilon, r, r_{0}}^{B^{\prime}}(\zeta) \mathrm{d} \zeta, \\
\mathscr{T}_{2}= & \int_{\mathbb{R}}\left(\operatorname{sign}_{\varepsilon}(\zeta-A(u)) \star J_{r_{0}} \otimes J_{r}\right) \partial_{t} R_{\varepsilon, r, r_{0}}^{B^{\prime}, \Delta x}(\zeta) \mathrm{d} \zeta, \\
\mathscr{T}_{3}= & \int_{\mathbb{R}}\left(\operatorname{sign}_{\varepsilon}\left(\zeta-A\left(u_{\Delta x}\right)\right) \star J_{r_{0}} \otimes J_{r}\right) \nabla \cdot R_{\varepsilon, r, r_{0}}^{g^{\prime}}(\zeta) \mathrm{d} \zeta, \\
\mathscr{T}_{4}= & \int_{\mathbb{R}}\left(\operatorname{sign}_{\varepsilon}(\zeta-A(u)) \star J_{r_{0}} \otimes J_{r}\right) \\
& \times\left(D_{+} \cdot R_{\varepsilon, r, r_{0}}^{G_{1}^{\prime}, \Delta x}(\zeta)+D_{-} \cdot R_{\varepsilon, r, r_{0}}^{G^{\prime}, \Delta x}(\zeta)\right) \mathrm{d} \zeta,
\end{aligned}
$$


where

$$
R_{\varepsilon, r, r_{0}}^{f}(\zeta)=R_{\varepsilon}^{f}(A(u), \zeta) \star J_{r_{0}} \otimes J_{r} \text { and } R_{\varepsilon, r, r_{0}}^{f, \Delta x}(\zeta)=R_{\varepsilon}^{f}\left(A\left(u_{\Delta x}\right), \zeta\right) \star J_{r_{0}} \otimes J_{r}
$$

for any function $f$, and $R_{\varepsilon}^{f}$ is defined in equation (4.5). Then

$$
\begin{aligned}
\left\|\mathscr{T}_{k}\right\|_{L^{\infty}\left(\Pi_{T}^{r_{0}}\right)} & \leq 8 \frac{\varepsilon}{\eta r_{0}}\left\|J^{\prime}\right\|_{\infty} \text { for } k=1,2, \\
\left\|\mathscr{T}_{3}\right\|_{L^{\infty}\left(\Pi_{T}^{r_{0}}\right)} & \leq 8 \frac{\varepsilon}{\eta r}\left\|J^{\prime}\right\|_{\infty} \sum_{i=1}^{d}\left\|f_{i}\right\|_{\text {Lip }}, \\
\left\|\mathscr{T}_{4}\right\|_{L^{\infty}\left(\Pi_{T}^{r_{0}}\right)} & \leq 8 \frac{\varepsilon}{\eta r}\left(1+\frac{\Delta x}{r}\right)\left\|J^{\prime}\right\|_{\infty} \sum_{k=1}^{2} \sum_{i=1}^{d}\left\|F_{k}^{i}\right\|_{\text {Lip }} .
\end{aligned}
$$

Proof. Consider $\mathscr{T}_{1}$. Moving the $t$ derivative onto $J_{r_{0}}$, we have that

$$
\mathscr{T}_{1}=\int_{\mathbb{R}} \operatorname{sign}_{\varepsilon}\left(\zeta-A\left(u_{\Delta x}\right)\right) R_{\varepsilon}^{B^{\prime}}(A(u), \zeta) \mathrm{d} \zeta \stackrel{\left(u_{\Delta x}, u\right)}{\star} J_{r_{0}} \otimes J_{r} \otimes \partial_{t} J_{r_{0}} \otimes J_{r} .
$$

By Lemma 5.5, equation (5.15), Lemma 5.7, and equation (5.20) with $f(z)=z$,

$$
\left\|\mathscr{T}_{1}\right\|_{L^{\infty}\left(\Pi_{T}^{r_{0}}\right)} \leq 4 \frac{\varepsilon}{\eta}\left\|J_{r_{0}} \otimes J_{r}\right\|_{L^{1}\left(\mathbb{R} \times \mathbb{R}^{d}\right)}\left\|\partial_{t} J_{r_{0}} \otimes J_{r}\right\|_{L^{1}\left(\mathbb{R} \times \mathbb{R}^{d}\right)} \leq 8 \frac{\varepsilon}{\eta r_{0}}\left\|J^{\prime}\right\|_{\infty} .
$$

The $L^{\infty}$ bound on $\mathscr{T}_{2}$ follows similarly.

Let us consider $\mathscr{T}_{3}$ :

$$
\mathscr{T}_{3}=\sum_{i=1}^{d} \int_{\mathbb{R}} \operatorname{sign}_{\varepsilon}\left(\zeta-A\left(u_{\Delta x}\right)\right) R_{\varepsilon}^{g_{i}^{\prime}}(A(u), \zeta) \mathrm{d} \zeta \stackrel{\left(u_{\Delta x}, u\right)}{\star} J_{r_{0}} \otimes J_{r} \otimes J_{r_{0}} \otimes \partial_{x_{i}} J_{r} .
$$

By Lemma 5.5, equation (5.15), Lemma 5.7, and equation (5.20) with $f(z)=f_{i}(z)$,

$$
\left\|\mathscr{T}_{3}\right\|_{L^{\infty}\left(\Pi_{T}^{r_{0}}\right)} \leq 4 \frac{\varepsilon}{\eta} \sum_{i=1}^{d}\left\|f_{i}\right\|_{\text {Lip }}\left\|\partial_{x_{i}} J_{r}\right\|_{L^{1}\left(\mathbb{R}^{d}\right)} \leq 8 \frac{\varepsilon}{\eta r}\left\|J^{\prime}\right\|_{\infty} \sum_{i=1}^{d}\left\|f_{i}\right\|_{\text {Lip }} .
$$

The terms in $\mathscr{T}_{4}$ are estimated in the same way, but in view (5.22) we can utilize the bound

$$
\left\|J_{r_{0}} \otimes D_{ \pm} J_{r}\right\|_{L^{1}\left(\mathbb{R} \times \mathbb{R}^{d}\right)} \leq 2\left\|J^{\prime}\right\|_{\infty} \frac{1}{r}\left(1+\frac{\Delta x}{r}\right) .
$$

\subsection{Concluding the Proof of Theorem 3.2}

Recall that $Q_{\varepsilon}, c f$. (5.1), was introduced as an approximation to the contraction functional $Q, c f$. (1.8). Recall the basic property $[10,33]$

$$
|u-v|=\int_{\mathbb{R}} Q(u, v ; \xi) \mathrm{d} \xi, \quad u, v \in \mathbb{R} .
$$

To argue for this relation, note that

$$
Q(u, v ; \xi)=|\chi(u ; \xi)|+|\chi(v ; \xi)|-2 \chi(u ; \xi) \chi(v ; \xi)=(\chi(u ; \xi)-\chi(v ; \xi))^{2} .
$$

Next, observe that

$$
\chi(u ; \xi)-\chi(v ; \xi)=\chi(u-v ; \xi-v)
$$


indeed, for any $S \in C_{0}^{1}(\mathbb{R})$,

$$
\begin{aligned}
\int_{\mathbb{R}} S^{\prime}(\xi)(\chi(u ; \xi)-\chi(v ; \xi)) \mathrm{d} \xi & \left.=\int_{u}^{v} S^{\prime}(\xi) \mathrm{d} \xi=\int_{0}^{u-v} S^{\prime}(\sigma+v) \mathrm{d} \sigma \quad \text { here } \sigma=\xi-v\right) \\
& =\int_{\mathbb{R}} S^{\prime}(\sigma+v) \chi(u-v ; \sigma) \mathrm{d} \sigma \\
& =\int_{\mathbb{R}} S^{\prime}(\xi) \chi(u-v ; \xi-v) \mathrm{d} \xi
\end{aligned}
$$

Hence, the claim follows:

$$
\int_{\mathbb{R}}(\chi(u ; \xi)-\chi(v ; \xi))^{2} \mathrm{~d} \xi=\int_{\mathbb{R}}|\chi(u-v ; \xi-v)| \mathrm{d} \xi=|u-v| .
$$

Let us quantify the approximation properties of $Q_{\varepsilon}$.

Lemma 5.18. Let $A^{\prime} \geq \eta>0, B=A^{-1}$, and $f=g \circ A$. Define

$$
\begin{aligned}
P & =\int_{\mathbb{R}} Q_{\varepsilon}(A(u), A(v) ; \zeta) B^{\prime}(\zeta) \mathrm{d} \zeta-|u-v| \\
M & =\int_{\mathbb{R}} Q_{\varepsilon}(A(u), A(v) ; \zeta) g^{\prime}(\zeta) \mathrm{d} \zeta-\operatorname{sign}(u-v)(f(u)-f(v)),
\end{aligned}
$$

and

$$
N=\int_{\mathbb{R}} Q_{\varepsilon}(A(u), A(v) ; \zeta) \mathrm{d} \zeta-|A(u)-A(v)|,
$$

for any $u$ and $v$, and where $Q_{\varepsilon}$ is given by (5.1). Then

$$
|P| \leq 16 \frac{\varepsilon}{\eta}, \quad|M| \leq 8 \frac{\varepsilon}{\eta}, \quad|N| \leq 8 \frac{\varepsilon}{\eta} .
$$

Proof. Because $A^{\prime}>0, Q(u, v ; \xi)=Q(A(u), A(v) ; A(\xi))$. Hence we can use (5.23) and a change of variables to obtain the identify

$$
P=\int_{\mathbb{R}}\left(Q_{\varepsilon}(A(u), A(v) ; \zeta)-Q(A(u), A(v) ; \zeta)\right) B^{\prime}(\zeta) \mathrm{d} \zeta .
$$

By definition of $Q$ and the equality $|\chi(u ; \xi)|=\operatorname{sign}(\xi) \chi(u ; \xi)$,

$$
Q(A(u), A(v) ; \zeta)=\operatorname{sign}(\zeta) \chi(A(u) ; \zeta)+\operatorname{sign}(\zeta) \chi(A(v) ; \zeta)-2 \chi(A(u) ; \zeta) \chi(A(v) ; \zeta) .
$$

Thus,

$$
\begin{aligned}
P= & \int_{\mathbb{R}}\left(\operatorname{sign}_{\varepsilon}(\zeta) \chi_{\varepsilon}(A(u) ; \zeta)-\operatorname{sign}(\zeta) \chi(A(u) ; \zeta)\right) B^{\prime}(\zeta) \mathrm{d} \zeta \\
& +\int_{\mathbb{R}}\left(\operatorname{sign}_{\varepsilon}(\zeta) \chi_{\varepsilon}(A(v) ; \zeta)-\operatorname{sign}(\zeta) \chi(A(v) ; \zeta)\right) B^{\prime}(\zeta) \mathrm{d} \zeta \\
& \left.+2 \int_{\mathbb{R}}\left(\chi(A(u) ; \zeta) \chi(A(v) ; \zeta)-\chi_{\varepsilon}(A(u) ; \zeta) \chi_{\varepsilon}(A(v) ; \zeta)\right)\right) B^{\prime}(\zeta) \mathrm{d} \zeta \\
= & : P_{1}+P_{2}+P_{3} .
\end{aligned}
$$

Finding that the measure of the support of the integrand is bounded by $4 \varepsilon$ for $P_{1}, P_{2}$, and $P_{3}$, we conclude that

$$
|P| \leq 16 \varepsilon\left\|B^{\prime}\right\|_{\infty},
$$


and then the bound on $P$ follows since $\left\|B^{\prime}\right\|_{\infty} \leq \eta^{-1}$.

To prove the inequality for $M$, note that

$$
\begin{aligned}
\operatorname{sign}(u-v)(f(u)-f(v)) & =\int_{\mathbb{R}} \operatorname{sign}(u-v)(\chi(u ; \zeta)-\chi(v ; \zeta)) f^{\prime}(\zeta) \mathrm{d} \zeta \\
& =\int_{\mathbb{R}}|\chi(u, \zeta)-\chi(v ; \zeta)| f^{\prime}(\zeta) \mathrm{d} \zeta \\
& =\int_{\mathbb{R}}[\operatorname{sign}(\zeta) \chi(u ; \zeta)+\operatorname{sign}(\zeta) \chi(v ; \zeta)-2 \chi(u ; \zeta) \chi(v ; \zeta)] f^{\prime}(\zeta) \mathrm{d} \zeta
\end{aligned}
$$

Changing variables, we arrive at

$$
\int_{\mathbb{R}} Q_{\varepsilon}(A(u), A(v) ; \zeta) g^{\prime}(\zeta) \mathrm{d} \zeta=\int_{\mathbb{R}} Q_{\varepsilon}(A(u), A(v) ; A(\zeta)) f^{\prime}(\zeta) \mathrm{d} \zeta
$$

and, since $\operatorname{sign}(\zeta) \chi(w ; \zeta)=\operatorname{sign}(A(\zeta)) \chi(A(w) ; A(\zeta))$, we find that

$$
\begin{aligned}
|M| \leq & \int_{\mathbb{R}}\left|\operatorname{sign}_{\varepsilon}(A(\zeta)) \chi_{\varepsilon}(A(u) ; A(\zeta))-\operatorname{sign}(A(\zeta)) \chi(A(u) ; A(\zeta))\right|\left|f^{\prime}(\zeta)\right| \mathrm{d} \zeta \\
& +\int_{\mathbb{R}}\left|\operatorname{sign}_{\varepsilon}(A(\zeta)) \chi_{\varepsilon}(A(v) ; A(\zeta))-\operatorname{sign}(A(\zeta)) \chi(A(v) ; A(\zeta))\right|\left|f^{\prime}(\zeta)\right| \mathrm{d} \zeta \\
& +2 \int_{\mathbb{R}}\left|\chi_{\varepsilon}(A(u) ; A(\zeta)) \chi_{\varepsilon}(A(v) ; A(\zeta))-\chi(A(u) ; A(\zeta)) \chi(A(v) ; A(\zeta))\right|\left|f^{\prime}(\zeta)\right| \mathrm{d} \zeta .
\end{aligned}
$$

Each of the three integrands is bounded by 2 and has support where $|A(\zeta)|<\varepsilon$, i.e., where $|\zeta| \leq \varepsilon / \eta$, hence $|M| \leq 8 \varepsilon / \eta$. The proof of the bound on $|N|$ is similar.

Concluding the Proof of Theorem 3.2. We shall choose a positive test function $\phi \leq 1$, such that $|\nabla \phi|$ and $|\Delta \phi|$ are bounded by $C \phi$. This will be convenient when we estimate terms containing $\nabla \phi$ or $\Delta \phi$.

A test function with the necessary properties can be defined as follows, fix $R>d \sqrt{d}$ and define $\hat{\phi}: \mathbb{R}^{d} \rightarrow \mathbb{R}$ by

$$
\hat{\phi}(x)= \begin{cases}1 & \text { if }|x| \leq R+\sqrt{d} \\ \exp ((R+\sqrt{d}-|x|) / \sqrt{d}) & \text { otherwise. }\end{cases}
$$

Define $\phi=\hat{\phi} \star J^{\otimes n}$ and note that $\phi(x)=1$ for $x \in B(0, R)$. Note that $\hat{\phi}$ is weakly differentiable and satisfies

$$
\partial_{x_{i}} \hat{\phi}= \begin{cases}-\frac{x_{i}}{\sqrt{d}|x|} \hat{\phi}(x) & |x|>R+\sqrt{d} \\ 0 & |x|<R+\sqrt{d} .\end{cases}
$$

It follows that $|\nabla \phi(x)| \leq \frac{1}{\sqrt{d}} \phi(x)$. In order to bound $\Delta \phi$ we first note that

$$
\Delta \hat{\phi}(x)=\left(\frac{1}{d}-\frac{d-1}{\sqrt{d}|x|}\right) \hat{\phi}(x), \text { for }|x|>R+\sqrt{d} .
$$

Furthermore,

$$
\frac{1}{d^{2}} \leq\left(\frac{1}{d}-\frac{d-1}{\sqrt{d}|x|}\right) \leq \frac{1}{d} \text { for }|x| \geq d \sqrt{d}
$$

It follows that $|\Delta \hat{\phi}| \leq \frac{1}{d} \hat{\phi}(x)$ whenever $|x|>R+\sqrt{d}$. Hence

$$
|\Delta \phi(x)| \leq \frac{1}{d} \phi(x) \text { for }|x|>R+2 \sqrt{d} .
$$


If $|x| \leq R+2 \sqrt{d}$ it follows by the lower bound $\phi(x) \geq e^{-2}$, that there exists a constant $C=C(d, J)$ such that $|\Delta \phi(x)| \leq C|\phi(x)|$.

The next lemma estimates how far $\left|u_{\Delta x}-u\right|$ is from it regularized counterpart

$$
\int B^{\prime}(\zeta)\left(\chi_{\varepsilon}\left(A\left(u_{\Delta x}\right) ; \zeta\right)-\chi_{\varepsilon}(A(u) ; \zeta)\right)^{2} \mathrm{~d} \zeta^{\left(u_{\Delta x}, u\right)} J_{r_{0}} \otimes J_{r} \otimes J_{r_{0}} \otimes J_{r} .
$$

Lemma 5.19. With the notation and assumptions of Lemma 5.1,

$$
\begin{gathered}
\int_{\mathbb{R}^{d}}\left|\int_{\mathbb{R}} B^{\prime}(\zeta) Q_{\varepsilon, r, r_{0}}\left(A\left(u_{\Delta x}\right), A(u) ; \zeta\right) \mathrm{d} \zeta-\right| u_{\Delta x}-u|| \phi \mathrm{d} x \leq C\left(r+r_{0}+\|\phi\|_{L^{1}\left(\mathbb{R}^{d}\right)} \frac{\varepsilon}{\eta}\right), \\
\int_{r_{0}}^{\tau} \int_{\mathbb{R}^{d}} \mid\left[\int_{\mathbb{R}} g^{\prime}(\zeta) Q_{\varepsilon, r, r_{0}}\left(A\left(u_{\Delta x}\right), A(u) ; \zeta\right) \mathrm{d} \zeta\right. \\
\left.\quad-\operatorname{sign}\left(u_{\Delta x}-u\right)\left(f(u)-f\left(u_{\Delta x}\right)\right)\right] \cdot \nabla \phi \mid \mathrm{d} x \mathrm{~d} t \leq C T\left(r+r_{0}+\|\phi\|_{L^{1}\left(\mathbb{R}^{d}\right)} \frac{\varepsilon}{\eta}\right),
\end{gathered}
$$

and

$$
\begin{aligned}
\int_{r_{0}}^{\tau} \int_{\mathbb{R}^{d}} \mid\left[\int_{\mathbb{R}} Q_{\varepsilon, r, r_{0}}\left(A\left(u_{\Delta x}\right), A(u) ; \zeta\right) \mathrm{d} \zeta\right. \\
\\
\left.\quad-\left|A\left(u_{\Delta x}\right)-A(u)\right|\right] \Delta \phi \mid \mathrm{d} x \mathrm{~d} t \leq C T\left(r+r_{0}+\|\phi\|_{L^{1}\left(\mathbb{R}^{d}\right)} \frac{\varepsilon}{\eta}\right)
\end{aligned}
$$

where the constant $C$ only depends on the initial data, $A$, and $f$.

Proof of Lemma 5.19. We establish (5.25) as follows:

$$
\begin{aligned}
& \int_{\mathbb{R}^{d}}\left|\int_{\mathbb{R}} B^{\prime}(\zeta) Q_{\varepsilon, r, r_{0}}\left(A\left(u_{\Delta x}\right), A(u) ; \zeta\right) \mathrm{d} \zeta-\right| u_{\Delta x}-u|| \phi \mathrm{d} x \\
&=\int_{\mathbb{R}^{d}} \mid \int_{0}^{T} \int_{\mathbb{R}^{d}}\left(\int_{\mathbb{R}} B^{\prime}(\zeta) Q_{\varepsilon}\left(A\left(u_{\Delta x}(s, y)\right), A(u(s, y)) ; \zeta\right) \mathrm{d} \zeta\right. \\
&\left.-\left|u_{\Delta x}(t, x)-u(t, x)\right|\right) J_{r_{0}}(t-s) J_{r}(x-y) \mathrm{d} y \mathrm{~d} s \mid \phi \mathrm{d} x \\
& \leq \int_{\mathbb{R}^{d}} \int_{0}^{T} \int_{\mathbb{R}^{d}} \mid \int_{\mathbb{R}^{\prime}} B^{\prime}(\zeta) Q_{\varepsilon}\left(A\left(u_{\Delta x}(s, y)\right), A(u(s, y)) ; \zeta\right) \mathrm{d} \zeta \\
&-\left|u_{\Delta x}(s, y)-u(s, y)\right| J_{r_{0}}(t-s) J_{r}(x-y) \mathrm{d} y \mathrm{~d} s \phi \mathrm{d} x \\
&+\int_{\mathbb{R}^{d}} \int_{0}^{T} \int_{\mathbb{R}^{d}}\left[u_{\Delta x}(t, x)-u_{\Delta x}(s, y)|+| u(t, x)-u(s, y) \mid\right] \\
& \times J_{r_{0}}(t-s) J_{r}(x-y) \mathrm{d} y \mathrm{~d} s \mathrm{~d} x \\
& \leq 16 \frac{\varepsilon}{\eta} \int_{\mathbb{R}^{d}} \phi \mathrm{d} x+2\left(\left|u_{0}\right|_{B V\left(\mathbb{R}^{d}\right)}+\left|u_{\Delta x}(0, \cdot)\right|_{B V\left(\mathbb{R}^{d}\right)}\right)\left(r+r_{0}\right) .
\end{aligned}
$$

The bounds (5.26) and (5.27) are proved in the same way. 
Writing the equation in Lemma 5.1 as

$$
\int_{\mathbb{R}} B^{\prime}(\zeta) \partial_{t} Q_{\varepsilon, r, r_{0}} \mathrm{~d} \zeta+\int_{\mathbb{R}} g^{\prime}(\zeta) \nabla Q_{\varepsilon, r, r_{0}} \mathrm{~d} \zeta=\int_{\mathbb{R}} \Delta Q_{\varepsilon, r, r_{0}} \mathrm{~d} \zeta+\mathcal{E}_{\varepsilon, r, r_{0}}^{\Delta x}
$$

we multiply by $\phi$, integrate over $t \in\left[r_{0}, \tau\right]$ where $r_{0}<\tau \leq T-r_{0}$, and integrate by parts in $x$, finally obtaining

$$
\begin{aligned}
\left.\int_{\mathbb{R}^{d}} \int_{\mathbb{R}} B^{\prime}(\zeta) Q_{\varepsilon, r, r_{0}} \mathrm{~d} \zeta \phi\right|_{t=r_{0}} ^{t=\tau} \mathrm{d} x-\int_{0}^{\tau} \int_{\mathbb{R}^{d}} \int_{\mathbb{R}} g^{\prime}(\zeta) Q_{\varepsilon, r, r_{0}} \cdot \nabla \phi \mathrm{d} \zeta \mathrm{d} x \mathrm{~d} t & =\int_{r_{0}}^{\tau} \int_{\mathbb{R}^{d}} \int_{\mathbb{R}} Q_{\varepsilon, r, r_{0}} \Delta \phi \mathrm{d} \zeta \mathrm{d} x \mathrm{~d} t+\int_{r_{0}}^{\tau} \int_{\mathbb{R}^{d}} \mathcal{E}_{\varepsilon, r, r_{0}}^{\Delta x} \phi \mathrm{d} x \mathrm{~d} t .
\end{aligned}
$$

Combining this with Lemma 5.19 gives

$$
\begin{aligned}
\left.\int_{\mathbb{R}^{d}}\left|u_{\Delta x}-u\right| \phi\right|_{t=r_{0}} ^{t=\tau} \mathrm{d} x-\int_{r_{0}}^{\tau} & \int_{\mathbb{R}^{d}} \operatorname{sign}\left(u_{\Delta x}-u\right)\left(f\left(u_{\Delta x}\right)-f(u)\right) \cdot \nabla \phi \mathrm{d} x \mathrm{~d} t \\
& \leq \int_{r_{0}}^{\tau} \int_{\mathbb{R}^{d}}\left|A\left(u_{\Delta x}\right)-A(u)\right| \Delta \phi \mathrm{d} x \mathrm{~d} t+\int_{r_{0}}^{\tau} \int_{\mathbb{R}^{d}} \mathcal{E}_{\varepsilon, r, r_{0}}^{\Delta x} \phi \mathrm{d} x \mathrm{~d} t+C_{T}\left(r+r_{0}+\frac{\varepsilon}{\eta}\right)
\end{aligned}
$$

where $C_{T}$ depends (linearly) on $T$. Using properties of $\phi$, this can be rewritten as

$$
\Lambda(\tau)-\Lambda\left(r_{0}\right) \leq C \int_{r_{0}}^{\tau} \Lambda(t) \mathrm{d} t+\overline{\mathcal{E}}_{\varepsilon, r, r_{0}}^{\Delta x, \tau}
$$

where

$$
\begin{aligned}
\Lambda(t) & =\int_{\mathbb{R}^{d}}\left|u_{\Delta x}(t, x)-u(t, x)\right| \phi(x) \mathrm{d} x, \\
\overline{\mathcal{E}}_{\varepsilon, r, r_{0}}^{\Delta x, \tau} & =\int_{r_{0}}^{\tau} \int_{\mathbb{R}^{d}} \mathcal{E}_{\varepsilon, r, r_{0}}^{\Delta x} \phi \mathrm{d} x \mathrm{~d} t+C_{T}\left(r+r_{0}+\frac{\varepsilon}{\eta}\right) .
\end{aligned}
$$

Gronwall's inequality then implies that

$$
\Lambda(\tau) \leq \Lambda\left(r_{0}\right)+\tau e^{C \tau}\left(\Lambda\left(r_{0}\right)+\overline{\mathcal{E}}_{\varepsilon, r, r_{0}}^{\Delta x, \tau}\right) .
$$

Recall that $u$ depends on $\eta$, and we now make this dependence explicit by writing $u^{\eta}$ and $\Lambda^{\eta}$. Our aim is to estimate $u_{\Delta x}-u^{0}$. By $(2.2)$,

$$
\begin{aligned}
\int_{B(0, R)} \mid & u_{\Delta x}(\tau, \cdot)-u^{0}(\tau, \cdot) \mid \mathrm{d} x \mathrm{~d} t-C \sqrt{\eta} \\
& \leq \Lambda^{\eta}(\tau) \\
& \leq C_{T}\left\|u_{\Delta x}\left(r_{0}, \cdot\right)-u^{0}\left(r_{0}, \cdot\right)\right\|_{L^{1}\left(\mathbb{R}^{d}\right)}+C_{T} \overline{\mathcal{E}}_{\varepsilon, r, r_{0}}^{\Delta x, \tau} \\
& \leq C r_{0}+\left\|u_{\Delta x}(0, \cdot)-u_{0}\right\|_{L^{1}\left(\mathbb{R}^{d}\right)}+C_{T} \overline{\mathcal{E}}_{\varepsilon, r, r_{0}}^{\Delta x, \tau} .
\end{aligned}
$$

Next, we estimate the terms in the integral of $\mathcal{E}_{\mathcal{\varepsilon}, r, r_{0}}^{\Delta x}$; these are the terms in (5.3)-(5.11). By Estimate 5.11,

$$
\iint_{\Pi_{T}^{r_{0}}} \mid \text { second term in (5.3)| } \mathrm{d} x \mathrm{~d} t \leq C \frac{\Delta x^{2}}{r^{3}}\left(1+\frac{\Delta x}{r}\right),
$$

where $C$ depends on the initial data. 
The integral of the terms (5.4)-(5.7) is bounded by Estimate 5.17:

$$
\iint_{\Pi_{T}^{r_{0}}}|(5.4)+\ldots+(5.7)| \mathrm{d} x \mathrm{~d} t \leq C \frac{\varepsilon}{\eta}\left(\frac{1}{r_{0}}+\frac{1}{r}\left(1+\frac{\Delta x}{r}\right)\right) .
$$

The integral of (5.8) is bounded by Estimate 5.15 as follows:

$$
\iint_{\Pi_{T}^{r_{0}}}|(5.8)| \mathrm{d} x \mathrm{~d} t \leq C \frac{\Delta x}{r}\left(1+\frac{\Delta x}{r}\right) .
$$

The integral of (5.9) is bounded using Estimate 5.13:

$$
\iint_{\Pi_{T}^{r_{0}}}|(5.9)| \mathrm{d} x \mathrm{~d} t \leq C \frac{\Delta x^{2}}{r^{3}}\left(1+\frac{\Delta x}{r}\right) .
$$

The term (5.11) is bounded using Estimates 5.8 and 5.9 (if $d>1$ ):

$$
\iint_{\Pi_{T}^{r_{0}}}|(5.11)| \mathrm{d} x \mathrm{~d} t \leq C \Delta x\left(\frac{1}{r^{2}}+\frac{1}{r}+\frac{1}{\varepsilon^{2} \sqrt{r_{0} r^{d}}}\right) .
$$

If $d=1$, we can use Estimate 5.10 to achieve the better bound

$$
\iint_{\Pi_{T}^{r_{0}}}\left|\lim _{\varepsilon \rightarrow 0}(5.11)\right| \mathrm{d} x \mathrm{~d} t \leq C \Delta x\left(\frac{1}{r^{2}}+\frac{1}{r}+\frac{1}{r_{0}}\right) .
$$

Finally, the term (5.10) is non-positive.

The fraction $\Delta x / r$ will turn out to be uniformly bounded (in fact vanishingly small), so we can overestimate it by a constant. Thus the bounds $(5.28)-(5.29 \mathrm{~b})$ give the following estimate for $\overline{\mathcal{E}}_{\varepsilon, r, r_{0}}^{\Delta x, \tau}$ :

$$
\overline{\mathcal{E}}_{\varepsilon, r, r_{0}}^{\Delta x, \tau} \leq C_{T}\left(r+r_{0}+\frac{\varepsilon}{\eta}+\frac{\varepsilon}{\eta r_{0}}+\frac{\varepsilon}{\eta r}+\frac{\Delta x}{r}+\frac{\Delta x}{r^{2}}+\frac{\Delta x}{\varepsilon^{2} \sqrt{r_{0} r^{d}}}\right) .
$$

If $u_{0} \in B V\left(\mathbb{R}^{d}\right),\left\|u_{\Delta x}(0, \cdot)-u_{0}\right\|_{L^{1}\left(\mathbb{R}^{d}\right)} \leq\left|u_{0}\right|_{B V\left(\mathbb{R}^{d}\right)} \Delta x$, so that

$$
\left\|u_{\Delta x}(\tau, \cdot)-u^{0}(\tau, \cdot)\right\|_{L^{1}(B(0, R))} \leq C_{T}\left(\Delta x+\sqrt{\eta}+r+r_{0}+\frac{\varepsilon}{\eta}+\frac{\varepsilon}{\eta r_{0}}+\frac{\varepsilon}{\eta r}+\frac{\Delta x}{r}+\frac{\Delta x}{r^{2}}+\frac{\Delta x}{\varepsilon^{2} \sqrt{r_{0} r^{d}}}\right) .
$$

Now we set $r=r_{0}=\sqrt{\eta}, \varepsilon=r^{4}$; using that $r<1$, the above simplifies to

$$
\left\|u_{\Delta x}(\tau, \cdot)-u^{0}(\tau, \cdot)\right\|_{L^{1}(B(0, R))} \leq C_{T}\left(r+\frac{\Delta x}{r^{\frac{17+d}{2}}}\right) .
$$

Finally, minimizing with respect to $r$ yields

$$
\left\|u_{\Delta x}(\tau, \cdot)-u^{0}(\tau, \cdot)\right\|_{L^{1}(B(0, R))} \leq C_{T} \Delta x^{\frac{2}{19+d}} .
$$

Remark 5.20. If $d=1$, the above estimate gives a convergence rate of $1 / 10$, which is better than the rate reported in [24]. However, when $d=1$, we can use (5.29b) instead of (5.29a). Then we have no terms with $\varepsilon$ in the denominator, so we can send $\varepsilon$ to zero in (5.2)-(5.11) before taking absolute values and integrating. Proceeding as above, i.e., setting $r=r_{0}=\sqrt{\eta}$, this yields the bound

$$
\left\|u_{\Delta x}(\tau, \cdot)-u^{0}(\tau, \cdot)\right\|_{L^{1}(B(0, R))} \leq C_{T}\left(r+\frac{\Delta x}{r^{2}}\right)
$$

which gives the rate $1 / 3[25]$. 


\section{WELL-POSEDNESS OF DIFFERENCE METHOD}

In this appendix we establish the well-posedness of the semi-discrete method. We also collect a series of a priori bounds.

Introduce

$$
\|\sigma\|_{1}=\Delta x^{d} \sum_{\alpha}\left|\sigma_{\alpha}\right| \quad \text { and } \quad|\sigma|_{B V}=\sum_{\alpha} \sum_{i=1}^{d}\left|\sigma_{\alpha+e_{i}}-\sigma_{\alpha}\right| .
$$

If these quantities are bounded we say that $\sigma=\left\{\sigma_{\alpha}\right\}$ is in $\ell^{1}\left(\mathbb{Z}^{d}\right)$ and of bounded variation. Let $u(t)=$ $\left\{u_{\alpha}(t)\right\}_{\alpha \in \mathbb{Z}^{d}}$ and $u_{0}=\left\{u_{\alpha}(0)\right\}_{\alpha \in \mathbb{Z}^{d}}$ and define the operator $\mathcal{A}: \ell^{1}\left(\mathbb{Z}^{d}\right) \rightarrow \ell^{1}\left(\mathbb{Z}^{d}\right)$ by

$$
(\mathcal{A}(u))_{\alpha}=\sum_{i=1}^{d} D_{-}^{i}\left[F^{i}\left(u_{\alpha}, u_{\alpha+e_{i}}\right)-D_{+}^{i} A\left(u_{\alpha}\right)\right] .
$$

Then (3.1) may be considered as the Cauchy's problem

$$
\left\{\begin{array}{l}
\frac{\mathrm{d} u}{\mathrm{~d} t}+\mathcal{A}(u)=0, \quad t>0 \\
u(0)=u_{0}
\end{array}\right.
$$

This problem has a unique continuously differentiable solution for small $t$, since $\mathcal{A}$ is Lipschitz continuous for each $\Delta x>0$. The solution defines a strongly continuous semigroup $\mathcal{S}(t)$ on $\ell^{1}$. We want to show that this semigroup is $\ell^{1}$ contractive. This follows by the theory presented in [14], given that $\mathcal{A}$ is accretive, i.e.,

$$
\sum_{\alpha} \operatorname{sign}\left(u_{\alpha}-v_{\alpha}\right)(\mathcal{A}(u)-\mathcal{A}(v))_{\alpha} \geq 0 .
$$

for any $u$ and $v$ in $\ell^{1}\left(\mathbb{Z}^{d}\right)[32,34]$.

Lemma A.1. The operator $\mathcal{A}: \ell^{1}\left(\mathbb{Z}^{d}\right) \rightarrow \ell^{1}\left(\mathbb{Z}^{d}\right)$ is accretive.

Proof. By definition

$$
(\mathcal{A}(u)-\mathcal{A}(v))_{\alpha}=\sum_{i=1}^{d} D_{-}^{i}\left[F^{i}\left(u_{\alpha}, u_{\alpha+e_{i}}\right)-F^{i}\left(v_{\alpha}, v_{\alpha+e_{i}}\right)-D_{+}^{i}\left(A\left(u_{\alpha}\right)-A\left(v_{\alpha}\right)\right)\right] .
$$

Let $\partial_{1} F^{i}$ and $\partial_{2} F^{i}$ denote the partial derivatives of $F^{i}$ with respect to the first and second variable respectively. Since $F^{i}$ is continuously differentiable there exist for each $(\alpha, i)$ some number $\tau_{\alpha, i}$ such that

$$
F^{i}\left(u_{\alpha}, u_{\alpha+e_{i}}\right)-F^{i}\left(v_{\alpha}, u_{\alpha+e_{i}}\right)=\partial_{1} F^{i}\left(\tau_{\alpha, i}, u_{\alpha+e_{i}}\right)\left(u_{\alpha}-v_{\alpha}\right)
$$

and similarly a number $\theta_{\alpha, i}$ such that

$$
F^{i}\left(v_{\alpha}, u_{\alpha+e_{i}}\right)-F^{i}\left(v_{\alpha}, v_{\alpha+e_{i}}\right)=\partial_{2} F^{i}\left(v_{\alpha}, \theta_{\alpha, i}\right)\left(u_{\alpha+e_{i}}-v_{\alpha+e_{i}}\right) .
$$

Let $w_{\alpha}=u_{\alpha}-v_{\alpha}$ then

$$
\begin{aligned}
F^{i}\left(u_{\alpha}, u_{\alpha+e_{i}}\right)-F^{i}\left(v_{\alpha}, v_{\alpha+e_{i}}\right) & =F^{i}\left(u_{\alpha}, u_{\alpha+e_{i}}\right)-F^{i}\left(v_{\alpha}, u_{\alpha+e_{i}}\right)+F^{i}\left(v_{\alpha}, u_{\alpha+e_{i}}\right)-F^{i}\left(v_{\alpha}, v_{\alpha+e_{i}}\right) \\
& =\partial_{1} F^{i}\left(\tau_{\alpha, i}, u_{\alpha+e_{i}}\right) w_{\alpha}+\partial_{2} F^{i}\left(v_{\alpha}, \theta_{\alpha, i}\right) w_{\alpha+e_{i}} .
\end{aligned}
$$

Let $A^{\prime}=a$. Then there exist some $\xi_{\alpha}$ such that

$$
A\left(u_{\alpha}\right)-A\left(v_{\alpha}\right)=a\left(\xi_{\alpha}\right) w_{\alpha} .
$$


Using these expressions we obtain

$$
\begin{aligned}
\sum_{\alpha} \operatorname{sign}\left(u_{\alpha}-v_{\alpha}\right)(\mathcal{A}(u)-\mathcal{A}(v))_{\alpha} \\
=\sum_{\alpha} \sum_{i=1}^{d} \operatorname{sign}\left(w_{\alpha}\right) D_{-}^{i}\left[\partial_{1} F^{i}\left(\tau_{\alpha, i}, u_{\alpha+e_{i}}\right) w_{\alpha}+\partial_{2} F^{i}\left(v_{\alpha}, \theta_{\alpha, i}\right) w_{\alpha+e_{i}}\right] \\
\quad-\sum_{\alpha} \sum_{i=1}^{d} \operatorname{sign}\left(w_{\alpha}\right) D_{-}^{i} D_{+}^{i}\left(a\left(\xi_{\alpha}\right) w_{\alpha}\right):=\mathscr{T}_{1}-\mathscr{T}_{2} .
\end{aligned}
$$

Consider $\mathscr{T}_{1}$ first. Since

$$
\begin{aligned}
D_{-}^{i}\left[\partial_{1} F^{i}\left(\tau_{\alpha, i}, u_{\alpha+e_{i}}\right) w_{\alpha}+\partial_{2} F^{i}\left(v_{\alpha}, \theta_{\alpha, i}\right) w_{\alpha+e_{i}}\right] & =\frac{1}{\Delta x}\left[\partial_{1} F^{i}\left(\tau_{\alpha, i}, u_{\alpha+e_{i}}\right) w_{\alpha}\right. \\
& \left.-\partial_{1} F^{i}\left(\tau_{\alpha-e_{i}, i}, u_{\alpha}\right) w_{\alpha-e_{i}}+\partial_{2} F^{i}\left(v_{\alpha}, \theta_{\alpha, i}\right) w_{\alpha+e_{i}}-\partial_{2} F^{i}\left(v_{\alpha-e_{i}}, \theta_{\alpha-e_{i}, i}\right) w_{\alpha}\right],
\end{aligned}
$$

it follows that

$$
\begin{aligned}
\mathscr{T}_{1}= & \frac{1}{\Delta x} \sum_{\alpha} \sum_{i=1}^{d}\left[\partial_{1} F^{i}\left(\tau_{\alpha, i}, u_{\alpha+e_{i}}\right)\left|w_{\alpha}\right|-\partial_{1} F^{i}\left(\tau_{\alpha-e_{i}, i}, u_{\alpha}\right) \operatorname{sign}\left(w_{\alpha}\right) w_{\alpha-e_{i}}\right. \\
& \left.+\partial_{2} F^{i}\left(v_{\alpha}, \theta_{\alpha, i}\right) \operatorname{sign}\left(w_{\alpha}\right) w_{\alpha+e_{i}}-\partial_{2} F^{i}\left(v_{\alpha-e_{i}}, \theta_{\alpha-e_{i}, i}\right)\left|w_{\alpha}\right|\right] \\
= & \frac{1}{\Delta x} \sum_{i=1}^{d}\left[\sum_{\alpha} \partial_{1} F^{i}\left(\tau_{\alpha, i}, u_{\alpha+e_{i}}\right)\left|w_{\alpha}\right|-\sum_{\alpha} \partial_{1} F^{i}\left(\tau_{\alpha, i}, u_{\alpha+e_{i}}\right) \operatorname{sign}\left(w_{\alpha+e_{i}}\right) w_{\alpha}\right. \\
& \left.+\sum_{\alpha} \partial_{2} F^{i}\left(v_{\alpha}, \theta_{\alpha, i}\right) \operatorname{sign}\left(w_{\alpha}\right) w_{\alpha+e_{i}}-\sum_{\alpha} \partial_{2} F^{i}\left(v_{\alpha}, \theta_{\alpha, i}\right)\left|w_{\alpha+e_{i}}\right|\right]
\end{aligned}
$$

Since each $F^{i}$ is monotone, it follows that $\mathscr{T}_{1} \geq 0$. Considering $\mathscr{T}_{2}$, we have

$$
\mathscr{T}_{2}=\frac{1}{\Delta x^{2}} \sum_{i=1}^{d} \sum_{\alpha}\left[a\left(\xi_{\alpha+e_{i}}\right) \operatorname{sign}\left(w_{\alpha}\right) w_{\alpha+e_{i}}-2 a\left(\xi_{\alpha}\right)\left|w_{\alpha}\right|+a\left(\xi_{\alpha-e_{i}}\right) \operatorname{sign}\left(w_{\alpha}\right) w_{\alpha-e_{i}}\right],
$$

from which it follows that $\mathscr{T}_{2} \leq 0$.

Lemma A.2. Suppose $F^{i}$ is monotone for each $1 \leq i \leq d$. For any positive $T$, there exists a unique solution $u=\left\{u_{\alpha}\right\}$ to $(3.1)$ on $[0, T]$ with the properties:

(i) $\|u(t)\|_{1} \leq\left\|u_{0}\right\|_{1}$.

(ii) For every $\alpha \in \mathbb{Z}^{d}$ and $t \in[0, T]$,

$$
\inf _{\beta}\left\{u_{\beta, 0}\right\} \leq u_{\alpha}(t) \leq \sup _{\beta}\left\{u_{\beta, 0}\right\}
$$

(iii) $|u(t)|_{B V} \leq\left|u_{0}\right|_{B V}$.

(iv) If $v=\left\{v_{\alpha}\right\}$ is a solution of the same problem with initial data $v_{0}$, then

$$
\|u(t)-v(t)\|_{1} \leq\left\|u_{0}-v_{0}\right\|_{1} .
$$

Proof. Parts (i), (iii) and (iv) follows since $\mathcal{S}(t)$ is a contraction semigroup. Part (ii) follows from [9].

Note that the $\ell^{1}$ bound in [(i)] implies that $u_{\alpha}(t)$ exists for all $t$, and not only for $t$ small. 
Lemma A.3. Suppose $F^{i}$ is monotone for each $1 \leq i \leq d$. If $u$ is a solution to $(3.1)$ and $\mathcal{A}\left(u_{0}\right) \in \ell^{1}\left(\mathbb{Z}^{d}\right)$, then for each $h>0$,

$$
\|u(t+h)-u(t)\|_{\ell^{1}} \leq\left\|\mathcal{A}\left(u_{0}\right)\right\|_{\ell^{1}} h .
$$

Proof. Suppose that $\left\|u^{\prime}(t)\right\| \leq C$. Then

$$
\|u(t+h)-u(t)\|=\left\|\int_{t}^{t+h} u^{\prime}(s) \mathrm{d} s\right\| \leq \int_{t}^{t+h}\left\|u^{\prime}(s)\right\| \mathrm{d} s \leq C h,
$$

and so Lipschitz continuity would follow. We claim that

$$
\frac{\partial}{\partial t}\left\|u^{\prime}(t)\right\| \leq 0
$$

Indeed,

$$
\begin{aligned}
\frac{\partial}{\partial t}\left\|u^{\prime}(t)\right\| & =\frac{\partial}{\partial t}\|\mathcal{A}(u(t))\| \\
& =\frac{\partial}{\partial t}\left[\Delta x^{d} \sum_{\alpha} \operatorname{sign}\left(\mathcal{A}(u(t))_{\alpha}\right) \mathcal{A}(u(t))_{\alpha}\right] \\
& =\Delta x^{d} \sum_{\alpha} \operatorname{sign}\left(\mathcal{A}(u(t))_{\alpha}\right) \partial_{t} \mathcal{A}(u \alpha(t))_{\alpha},
\end{aligned}
$$

and

$$
\begin{aligned}
\partial_{t} \mathcal{A}(u(t))_{\alpha}= & \frac{\partial}{\partial t} \sum_{i=1}^{d} D_{-}^{i}\left[F^{i}\left(u_{\alpha}(t), u_{\alpha+e_{i}}(t)\right)-D_{+}^{i} A\left(u_{\alpha}(t)\right)\right] \\
= & \sum_{i=1}^{d} D_{-}^{i}\left[\partial_{1} F^{i}\left(u_{\alpha}(t), u_{\alpha+e_{i}}(t)\right) u_{\alpha}^{\prime}(t)+\partial_{2} F^{i}\left(u_{\alpha}(t), u_{\alpha+e_{i}}(t)\right) u_{\alpha+e_{i}}^{\prime}(t)\right] \\
& -\sum_{i=1}^{d} D_{-}^{i} D_{+}^{i} a\left(u_{\alpha}(t)\right) u_{\alpha}^{\prime}(t) \\
= & -\sum_{i=1}^{d} D_{-}^{i}\left[\partial_{1} F^{i}\left(u_{\alpha}(t), u_{\alpha+e_{i}}(t)\right) \mathcal{A}(u(t))_{\alpha}+\partial_{2} F^{i}\left(u_{\alpha}(t), u_{\alpha+e_{i}}(t)\right) \mathcal{A}(u(t))_{\alpha+e_{i}}\right] \\
& +\sum_{i=1}^{d} D_{-}^{i} D_{+}^{i} a\left(u_{\alpha}(t)\right) \mathcal{A}(u(t))_{\alpha} .
\end{aligned}
$$

Considering the similarity between this computation and (A.1), it is seen that (A.2) holds. We conclude that $\left\|u^{\prime}(t)\right\| \leq\left\|\mathcal{A}\left(u_{0}\right)\right\|$ and so the lemma follows.

\section{REFERENCES}

[1] B. Andreianov, M. Bendahmane and K.H. Karlsen, Discrete duality finite volume schemes for doubly nonlinear degenerate hyperbolic-parabolic equations. J. Hyperbolic Differ. Equ. 7 (2010) 1-67.

[2] B. Andreianov and N. Igbida, On uniqueness techniques for degenerate convection-diffusion problems. Int. J. Dyn. Syst. Differ. Eq. 4 (2012) 3-34.

[3] D. Aregba-Driollet, R. Natalini and S. Tang, Explicit diffusive kinetic schemes for nonlinear degenerate parabolic systems. Math. Comput. 73 (2004) 63-94.

[4] G. Barles and E.R. Jakobsen, Error bounds for monotone approximation schemes for Hamilton-Jacobi-Bellman equations. SIAM J. Numer. Anal. 43 (2005) 540-558. 
[5] F. Bouchut and B. Perthame, Kružkov's estimates for scalar conservation laws revisited. Trans. Amer. Math. Soc. 350 (1998) $2847-2870$.

[6] F. Bouchut, F. R. Guarguaglini and R. Natalini, Diffusive BGK approximations for nonlinear multidimensional parabolic equations. Indiana Univ. Math. J. 49 (2000) 723-749.

[7] L.A. Caffarelli and P. E. Souganidis. A rate of convergence for monotone finite difference approximations to fully nonlinear, uniformly elliptic PDEs. Commun. Pure Appl. Math. 61 (2008) 1-17.

[8] J. Carrillo, Entropy solutions for nonlinear degenerate problems. Arch. Ration. Mech. Anal. 147 (1999) $269-361$.

[9] A. Chambolle and B.J. Lucier, Un principe du maximum pour des opérateurs monotones. C. R. Acad. Sci. Paris Sér. I Math. 326 (1998) 823-827.

[10] G.-Q. Chen and B. Perthame, Well-posedness for non-isotropic degenerate parabolic-hyperbolic equations. Ann. Inst. Henri Poincaré, Anal. Non Linéaire 20 (2003) 645-668.

[11] G.-Q. Chen and K.H. Karlsen, Quasilinear anisotropic degenerate parabolic equations with time-space dependent diffusion coefficients. Commun. Pure Appl. Anal. 4 (2005) 241-266.

[12] G.-Q. Chen and K.H. Karlsen, $L^{1}$-framework for continuous dependence and error estimates for quasilinear anisotropic degenerate parabolic equations. Trans. Amer. Math. Soc. 358 (2006) 937-963.

[13] B. Cockburn, Continuous dependence and error estimation for viscosity methods. Acta Numer. 12 (2003) $127-180$.

[14] M.G. Crandall and T.M. Liggett, Generation of semi-groups of nonlinear transformations on general Banach spaces. Amer. J. Math. 93 (1971) 265-298.

[15] M.G. Crandall and P.-L. Lions, Two approximations of solutions of Hamilton-Jacobi equations. Math. Comput. 43 (1984) $1-19$.

[16] C.M. Dafermos, Hyperbolic conservation laws in continuum physics. Vol. 325 of Grundl. Math. Wiss. [Fundamental Principles of Mathematical Sciences]. 3rd edition Springer-Verlag, Berlin (2010).

[17] S. Evje and K.H. Karlsen, Discrete approximations of BV solutions to doubly nonlinear degenerate parabolic equations. Numer. Math. 86 (2000) 377-417.

[18] S. Evje and K.H. Karlsen, Monotone difference approximations of BV solutions to degenerate convection-diffusion equations. SIAM J. Numer. Anal. 37 (2000) 1838-1860.

[19] S. Evje and K.H. Karlsen, An error estimate for viscous approximate solutions of degenerate parabolic equations. J. Nonlin. Math. Phys. 9 (2002) 262-281.

[20] R. Eymard, T. Gallouët and R. Herbin, Error estimate for approximate solutions of a nonlinear convection-diffusion problem. Adv. Differ. Equ. 7 (2002) 419-440.

[21] R. Eymard, T. Gallouët, R. Herbin and A. Michel, Convergence of a finite volume scheme for nonlinear degenerate parabolic equations. Numer. Math. 92 (2002) 41-82.

[22] H. Holden, K.H. Karlsen, K.-A. Lie and N.H. Risebro, Splitting methods for partial differential equations with rough solutions. EMS Series of Lect. Math. Analysis and MATLAB programs. European Mathematical Society (EMS), Zürich (2010).

[23] K.H. Karlsen and N.H. Risebro, Convergence of finite difference schemes for viscous and inviscid conservation laws with rough coefficients. ESAIM: M2AN 35 (2001) 239-269.

[24] K.H. Karlsen, U. Koley N.H. Risebro, An error estimate for the finite difference approximation to degenerate convectiondiffusion equations. Numer. Math. 121 (2012) 367-395.

[25] K.H. Karlsen, N.H. Risebro E.B. Storrøsten, $L^{1}$ error estimates for difference approximations of degenerate convection-diffusion equations. Math. Comput. 83 (2014) 2717-2762.

[26] S.N. Kružkov, First order quasilinear equations with several independent variables. Mat. Sb. (N.S.) 81 (1970) $228-255$.

[27] N.V. Krylov, The rate of convergence of finite-difference approximations for Bellman equations with Lipschitz coefficients. Appl. Math. Optim. 52 (2005) 365-399.

[28] N.N. Kuznecov, The accuracy of certain approximate methods for the computation of weak solutions of a first order quasilinear equation. Ž. Vyčisl. Mat. i Mat. Fiz. 16 (1976) 1489-1502, 1627.

[29] P.-L. Lions, B. Perthame and E. Tadmor, A kinetic formulation of multidimensional scalar conservation laws and related equations. J. Amer. Math. Soc. 7 (1994) 169-191.

[30] C. Makridakis and B. Perthame, Optimal rate of convergence for anisotropic vanishing viscosity limit of a scalar balance law. SIAM J. Math. Anal. 34 (2003) 1300-1307.

[31] M. Ohlberger, A posteriori error estimates for vertex centered finite volume approximations of convection-diffusion-reaction equations. ESAIM: M2AN 35 (2001) 355-387.

[32] N.H. Pavel, Differential equations, flow invariance and applications. Vol. 113 of Res. Notes Math. Pitman (Advanced Publishing Program), Boston, MA (1984).

[33] B. Perthame, Uniqueness and error estimates in first order quasilinear conservation laws via the kinetic entropy defect measure. J. Math. Pures Appl. 77 (1998) 1055-1064.

[34] K. Sato, On the generators of non-negative contraction semigroups in Banach lattices. J. Math. Soc. Japan 20 (1968) $423-436$.

[35] A.I. Vol'pert and S.I. Hudjaev, The Cauchy problem for second order quasilinear degenerate parabolic equations. Mat. Sb. (N.S.) $\mathbf{7 8}$ (1969) 374-396.

[36] Z.Q. Wu and J.X. Yin, Some properties of functions in $B V_{x}$ and their applications to the uniqueness of solutions for degenerate quasilinear parabolic equations. Northeast. Math. J. 5 (1989) 395-422. 